Portland State University

PDXScholar

$1-1-1976$

\title{
Theoretical studies in cooperative phenomena and population ecology
}

Shripad Tuljapurkar

Portland State University

Follow this and additional works at: https://pdxscholar.library.pdx.edu/open_access_etds Let us know how access to this document benefits you.

Recommended Citation

Tuljapurkar, Shripad, "Theoretical studies in cooperative phenomena and population ecology" (1976). Dissertations and Theses. Paper 598.

https://doi.org/10.15760/etd.598

This Dissertation is brought to you for free and open access. It has been accepted for inclusion in Dissertations and Theses by an authorized administrator of PDXScholar. Please contact us if we can make this document more accessible: pdxscholar@pdx.edu. 
THEORETICAL STUDIES IN COOPERATIVE

PHENOMENA AND POPULATION FCOLOGY

by

SHRIPAD D. TULJAPURKAR

A thesis submitted in partial fulfillment of the requirements for the degree of

\author{
DOCTOR OF PHILOSOPHY \\ in \\ ENVIRONMENTAL SCIENCES \\ AND RESOURC.ES - PHYSICS
}

Portland State Iniversity

1976

(c) Shripad Digambar Tuljapurkar 1976 
TO THE OFFICE OF GRADUATE STUDIES AND RESEARCH:

The members of the Committee approve the thesis of

Shripad D. Tuljapurkar presented November 24, 1976.

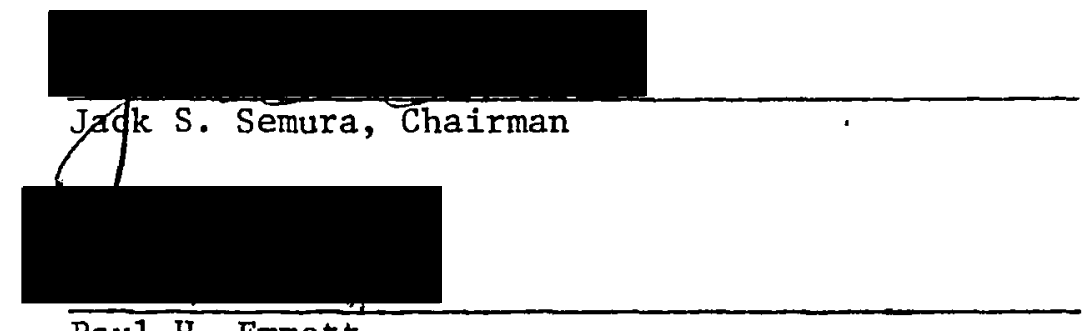

Pau1 H. Emmett

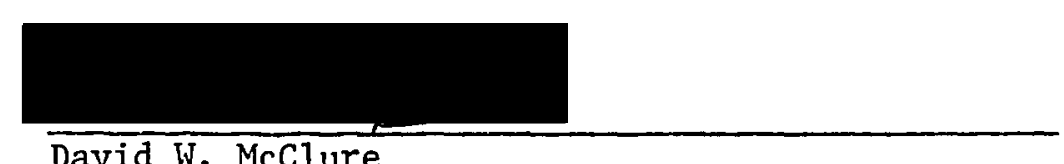

David W. McClure

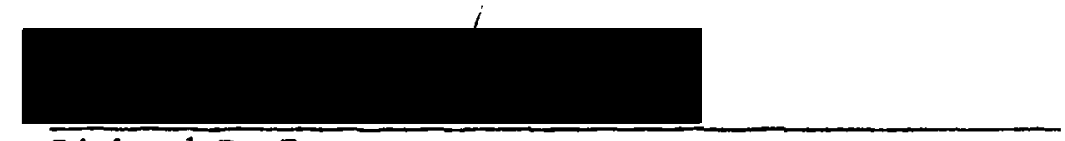

Richard R. Petersen

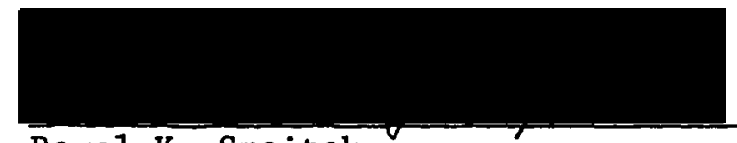

Pave1 K. Smejtek

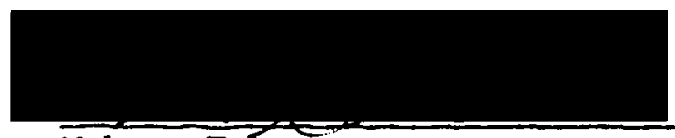

Makoto Takeo

APPROVED:

Mark Gurevitch, Head, Department of Physics

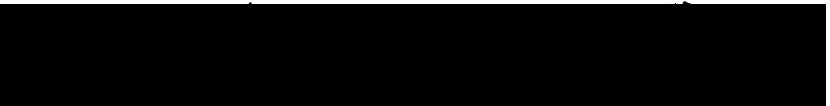

Stanley E. Rauch, Dean of Graduate Studies and Research 
AN ABSTRACT OF THE THESIS OF Shripad D. Tuljapurkar for the Doctor of Philosophy in Environmental Sciences and Resources - Physics presented November $24,1976$.

Title: Theoretical Studies in Cooperative Phenomena and Population Ecology

APPROVED BY MEMBERS OF THE THESIS COMMITTEE:

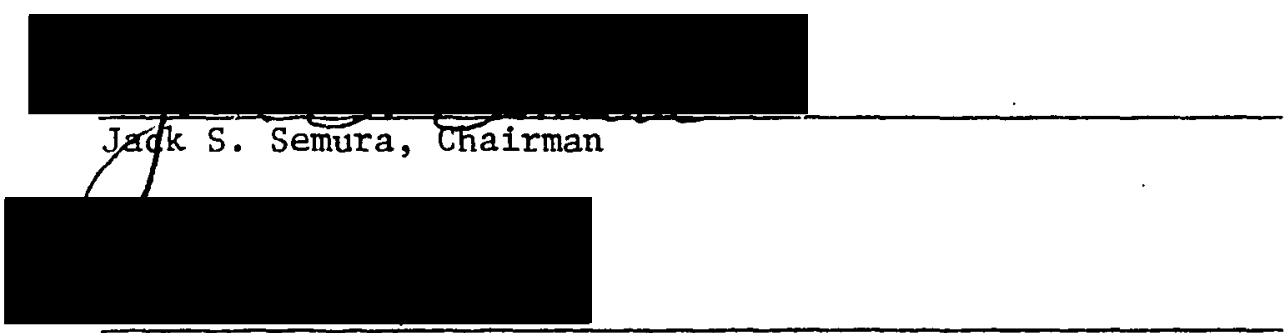

Paul H. Enmett

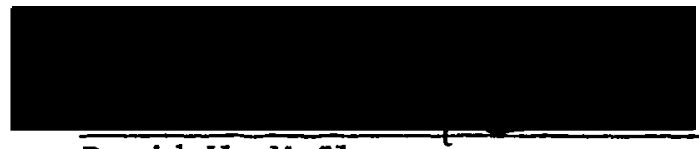

David W. McClure

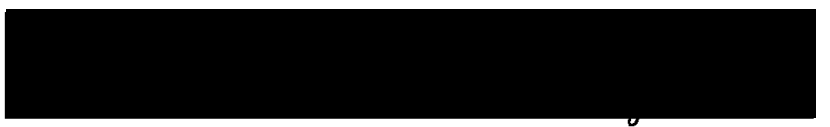

Richard R. Petersen

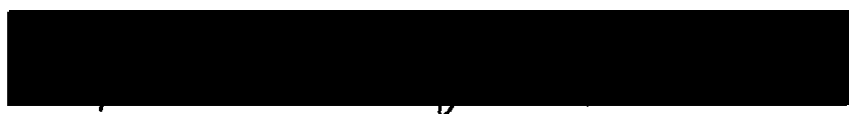

Pavel K. Smejtek

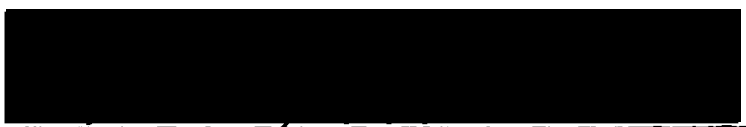

Makoto Takeo 
We study problems in the stability of nonlinear ecological models and in the theory of collective motion in physical systems. We first establish criteria for global stability in deterministic nonlinear population models, including the most general criteria so far available for the Lotka-Volterra model. Next we study conditions for coexistence under periodic perturbations in population models and establish criteria for the appearance of dynamic equilibrium states. The third study in ecological stability establishes that a measure of the stability of population models in the presence of white noise is given by a Liapunov function for the nonlinear deterministic model, and the implications of the result are examined. We consider next the use of kinetic equations to study physical systems, and prove that the use of higher order derivatives in the Mori formalism leads to results formally identical with Mori's continued fraction theory. We then apply the method of using higher derivatives to develop a physical picture of collective mode dynamics in the linear Heisenberg chain. The collective modes and their time scales are isolated and studied. 


\section{ACKNOWLEDGEMENTS}

This thesis is dedicated to my mother and my late father for their love, inspiration and support, and to my wife Shubha who has sustained me through the frustrations of the past four years. I am especially grateful to my friend and teacher Dr. Jack Semura. He has been tolerant of my more far-fetched ideas, and in many patient hours as a listener and critic he has taught me much about research and the nature of science. I wish to acknowledge Dr. R. P. Singh of the Indian Institute of Technology, Bombay for my first insight into the beauty and power of theoretical physics.

I thank Dr. Mark Gurevitch, Dr. Makoto Takeo, Dr. Bob Rempfer, and Mrs. M. Dawn Dressler for their advice and continual support. I have had the privilege of being treated more as a friend and colleague than as a student, and for this I am grateful to many people but most especially to Dr. Ray Sommerfeldt. Many thanks are due my good frrlends Mr. Pradip Rathod and Mr. Pramod Karulkar for the happy times we have shared.

I have had invaluable assistance, sympathy and great good cheer from Mrs. Jan Onchi and Ms. Virginia Pfaff. I will always be grateful to Mrs. Ellen Garrison whose optimism and efficiency have kept this thesis on schedule, and whose careful typing makes the pages which follow look elegant. Finally I thank Dr. J. J. Kohut, Mr. R. Lockerby and Ms. K. Roley for their kindness and cooperation. 
TABLE OF CONTENTS

PAGF.

ACKNOWLEDGEMENTS . . . . . . . . . . . . . . .

LIST OF FIGURES . . . . . . . . . . . . . . . . . vii

CHAPTER

I INTRODUCTION . . . . . . . . . . . . . . 1

II STABILITY IN ECOLOGICAL MODELS : AN OVERVIEW . . . 6

III GLOBAL STABILITY IN SIMPLE ECOLOGICAL MODELS . . . 13

Introduction ............. 13

A General Stability Theorem . . . . . . 15

A Special Case............. . . 18

Two-Species Lotka-Volterra Models . . . . 19

Many-Species Models . . . . . . . . 22

D-Stability

Qualitative Stability

Sufficiency Conditions: The Symmetric-Part Test 25

Discussion . . . . . . . . . . 26

IV DYNAMTC EQUILIBRIUM IN SIMPLE ECOLOGICAL MODELS • 27

Introduction . . . . . . . . . . 27

Convergence of Trajectories . . . . . . . 29

Equilibrium Under Periodic Perturbations . . . 32

Dynamic Equilibrium in General Models . . . . 41

Theory

General Models 
Discussion .. . . . . . . . . . 49

V STOCHASTIC STABILITY AND LIAPUNOV STABILITY . • . 53

Introduction ............ . . 53

The Nature of Noise . . . . . . . . 55

Time Scales and Models

Stochastic Population Models Involving Noise

Stability in Models With White Noise . . . 60

Adding White Noise to Models

Boundary Conditions

A Solution Procedure

A Liapunov Function

An Fxample

Liapunov Functions: Geometry and Stability .

The Geometrical View of Stability

Geometrical Pitfalls: An Example

Discussion . . . . . . . . . . . .

VI KINETIC EQUATIONS . . . . . . . . . . . 90

Introduction . . . . . . . . . . 90

Mori's Theory ............. . 91

Extended Derivatives . . . . . . . . 95

VII COLLECTIVE MODES IN THE FERROMAGNETIC HEISFNBERG

CHAIN . . . . . . . . . . . . . . 102

Introduction . . . . . . . . . . 102

Dynamical Variables . . . . . . . . . 104

Two-Variable Theory . . . . . . . . 107

Three Variable Theory . . . . . . . . 112

Discussion ............. . . 118

REFERENCES ..................... 120 
APPEIDIX A . . . . . . . . . . . . . . . . 126

APPENDIX B............................... 129

APPENDIX C . . . . . . . . . . . . . . . . . . . . 131 


\section{LIST OF FIGURES}

\section{FIGURE}

1. Representation of the action of the contraction

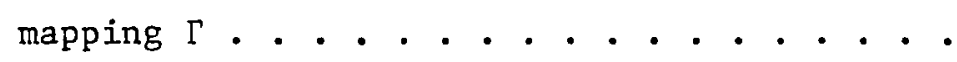

2. Biomass ratios for successive iteration steps using the Lotka-Volterra mode1 . . . . .

3. Plots of parameter space for the Lotka-Volterra mode1 of two-species competition ..... .

4. Biomass ratios for the competition model of Maynard Smith ............. .

5. Biomass ratios for Leslie's predator-prey model.

6. Contours of constant value for $\phi_{1}$. . . . . . 80

7. Contours of constant value for $\phi_{2}$. . . . . . 81

8. Contours of constant value for $\phi_{3}$. . . . . . 82

9. Contours of constant value for $\phi_{4}$. . . . . 83 
CHAPTER I

INTRODUCTION

This thesis describes the results of theoretical studies in the areas of mathematical ecology and collective phenomena in physical systems. In Chapter II we present an overview of our work on the stability of nonlinear population models. Results concerning the stability of deterministic population models are set out in Chapters III and IV, while Chapter $\mathrm{V}$ presents an analysis of stochastic population models. We study the stochastic description of physical systems and present results on kinetic equations in Chapter VI. These latter results are applied to the study of collective mode dynamics in linear Heisenberg magnets in Chapter VII.

The work we describe here on ecological stability theory contributes to an understanding of the mathematical and analytical questions which are essential to the successful development of a quantitative ecology. Our work on one-dimensional magnets contributes to an understanding of the magnetic properties of effectively one-dimensional organic chains. These organic chains are currently of considerable interest as potential high-temperature superconductors. The questions examined in this thesis are thus relevant to the development of environmental resource-management science and energy-related materials science.

The problems examined in this thesis, whether embedded in 
ecological or physical contexts, have a common conceptual and methodological basis. This basis lies in the use of statistical mechanics to describe nonequilibrium systems and in the use of coarse-graining arguments to reduce the statistical formalism to explicit computational forms in particular cases. In the course of the work described in this thesis we have found that the common basis just defined is both conceptually and practically valuable in the study of physical and ecological problems. In the rest of this chapter we discuss some aspects of this basis in a qualitative fashion.

The specific systems studied in the chapters which follow are examples of many-component interacting systems which are generally constrained by interactions with external systems, i.e., an environment of some sort. In order to study the dynamics of any many-component system it is usually assumed that one can set down a large enough set of state variables and dynamical equations which describe the evolution of these state variables. The set of state variables describes the system and each component completely, and the dynamical equations ensure that one can follow the evolution of the system over time. Given that many components are to be described it is natural to use statistical mechanics and consider the system to be described by a probability density in the phase space of the state variables. The time evolution of this probability density may then be determined from the dynamical equations. At this stage the problem is completely formulated but is nevertheless usually intractable because of the number of variables and the nature of the dynamical equations.

One therefore considers the system from an observational 
standpoint. Observations are usually made of spatial and temporal responses to external probes which are determined by collective behavior, and observations are usually 'coarse-trained', i.e., there is a range of time-scales and spatial scales which is probed, while dynamical changes outside these scales are not directly measured. The coarse nature of observations suggests a coarse-graining of the dynamical specification of the system. Thus one focuses selectively on those state-variables whose temporal and spatial variability falls into a macroscopically observable range. Next one examines the dynamical equations obeyed by this smaller set of variables and simplifies the equations by treating the more rapidly fluctuating variables stochastically and the very slowly varying quantities as effectively constant. The resulting simplified equations now provide a hopefully tractable, macroscopic description of the system.

The program outlined above has evolved from work in nonlinear mechanics (Krylov and Bogoliubov 1943), statistical mechanics (Kubo 1963, Lax 1966, Mori 1965a, b, Wilson 1975), control theory (Stratonovitch 1966), and applied mathematics (Griego and Hersh 1971, Papanicolau and Kohler 1974). From a mathematical standpoint several reductions of the type we have discussed have been carried out. In most cases it is assumed that the complete description which one starts with is a nonlinear Markov system. This seems reasonable since it may be possible to eliminate non-Markov terms by expanding the set of state variables, and has been an effective assumption in physical problems (Penrose 1970). The nonlinear Markov system is then reduced to one of several useful forms. Examples in our work are: diffusion Markov processes 
(Chapter V), linear non-Markov process (Chapter VI), nonlinear nonMarkov processes (Chapter VII). These three types of reduction also occur in the study of critical phenomena and mode-coupling theory (Kawasaki 1975), physical and chemical systems far from equilibrium (Kubo et. al. 1974, Haken 1975).

It is useful to emphasize that the general program of reduction outlined above is applicable to nonphysical systems just as to physical ones. In physics the specification of a state space and a dynamical equation can immediately be written down using the Hamiltonian forma1ism. Additionally the direct applicability of microscopic conservation laws provides useful constraints on the dynamics. For ecological systems on the other hand, there is no general method available for setting up state variables. Consequently it is necessary to explore the nature of different specifications in the way we have done in Chapters III to $\mathrm{V}$.

One other feature of physical systems which is very useful is the availability of natural scale factors such as system size or particle number. The natural scale factors for ecological systems are not yet known in any general way. Natural scale factors enter into the program of reduction in two ways. First natural scale factors provide natural perturbation parameters for the analysis of fluctuations. An example of such a perturbation parameter is system volume which plays an essential role in the system size expansion of the master equation for nonequilibrium physical systems (Kubo et. a1. 1974, Van Kampen 1965). We have utilized the strength of external fluctuations in a similar way in Chapter $\checkmark$. Second there is the technique of scallng dynamical variables 
in order to study scale-invariant dynamics (Mori 1974). Given the vast range of spatial and temporal scales that are involved in ecological systems (see the discussion of ecological time scales in Chapter V) it seems likely that it would be useful to study scale invariance in biological systems. The systematic application of a program of the sort we have outlined should be a useful component of progress in the understanding of nonequilibrium systems. 
CHAPTER II

STABILITY IN ECOLOGICAL MODELS: AN OVERVIEW

We set out here the broad outlines of our investigation of nonlinear dynamical models generally and ecological models in particular. The emphasis in our work is on the mathematical aspects of models which are extensively employed by ecologists. As is discussed below, the models we study arise naturally in analyses of a very wide range of physical and nonphysical (ecological, economic, and social) problems. Therefore, insights that emerge from our studies of models are in fact applicable to a range of problems in addition to ecological ones. However, we have studied mathematical questions which are embedded in an ecological context and have tried to translate results on the mathemat1cal behavior of models into the same general context.

The importance of seeking mathematical insights into ecological models can be brought out clearly by an example. Several authors, including Elton (1958) and MacArthur (1955), have argued that stability in ecosystems ought to increase with the number of links between species in a trophic web. However, May (1974) has analyzed the mathematical stability of a number of models of interacting populations, and has demonstrated that increasing complexity does not necessarily lead to an increase in mathematical stability. This demonstration has led to an ongoing re-examination of the meaning of the diversity-stability relationship (Pielou 1975). Examples such as this suggest that the 
mathematics of ecological models is indeed a matter of relevance to the development of ecological theory. It is certainly true that the use of models in the study of a specific ecological system may not be rewarding or meaningful in the absence of detalled flrst-hand knowledge of the ecological realities. However, it is equally true that an understanding of the mathematical characteristics of the models which may be used in ecology is an essential prerequisite to the development of quantitative ecology. A careful mathematical analysis may often shed 1 ight on what seems to be a puzzling yet general feature of real ecosystems (for example, May's (1975) demonstration that empirical 'laws' concerning species-abundance relationships may be actually artifacts of the mathematical distribution used to fit the data).

The relationship of the traditions and methods of theoretical physics to the development of quantitative ecology is well known. To quote Holling (1973): ". . . traditions of analysis in theoretical and empirical ecology have been largely inherited from developments in classical physics and its applied variants". Although Holling goes on to call for new developments in quantitative ecology, it happens that considerable recent work in physics is very similar in spirtt and technique to new developments in theoretical ecology. Examples are found in the work of Goel et. al. (1971) on population models, Goodwin (1970) on bacterial growth cycles, Kerner (1964) and Leigh (1971) on population models, and Margalef (1958) and Pielou (1975) on ecological diversity, all of which draw on the machinery of equilibrium statistical mechanics: The analysis we present of stochastic population models in Chapter $V$ draws extensively on ideas and methods which have been used to study the 
spiking of lasers (Takagahara 1976), critical fluctuations near phase transitions (Haken 1976), and a range of other collective phenomena in physics (Kubo et. a1. 1974, Mori 1965, Wilson 1975). In fact, the methods of Chapter $V$ relate directly to the analysis of nonlinear physical systems, and our results are applicable to physical problems (Mortensen 1969, Papanicolau 1973). Yet another example from the work reported here is the study of stability in Chapter III, which is applicable to ecological models (Levins 1974), chemical systems far from equilibrium (Field and Noyes 1973), and competitive economic equilibria (Siljak 1976). These examples make it clear that current research in theoretical physics can contribute to analytical and mathematical questions about ecological models.

The work reported in the next three chapters of this thesis is a study of various types of mathematical stability in ecological models. The concept of stability plays an important role in syntheses of ecological theory (MacArthur 1970, Pi_iou 1969, Slobodkin 1961, Planka 1973, Ricklefs 1973, Poole iy74, Maynard Smith 1974, May 1974), and is ex-

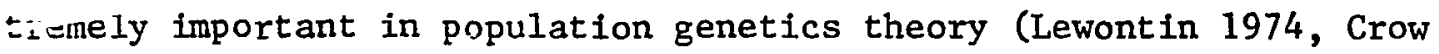
and Kimura 1970, Wright 1973). However, in the ecological literature the precise meaning of stability has long been undefined and a multiplicity of stability concepts are to be found. Two papers taken from a common source illustrate the situation: Preston (1969) discusses empirically and qualitatively the nature of ecological stability; Lewontin (1969) defines very precisely several types of mathematical stability and their possible relationships to ecological stability. Our concern is with mathematical stability since any quantification of 
ecological stability will require the definttion of stability in mathematical terms.

Three basic types of mathematical stability are examined in this thesis, all in relation to continuous nonlinear differential equation models of interacting populations. In large part our studies concentrate on robust or generic mathematical features of ecological models, and therefore, many of our results are applicable to a very large group of continuous nonlinear ecological models. The value of generic results is discussed by several authors (Levins 1966, May 1975, MacArthur 1970) and should be clear in the context of our work.

The three types of stability we examine are as follows. First, stabllity about a single equilibrium point. This type of stability might almost be called the classical type, and dates from the work of Volterra (1927), Lotka (1954), Gause (1934), while it was perhaps most strongly advocated by MacArthur (1969, 1970, 1972). Second, stability about a dynamic equilibrium state (alternatively, a state that might be classified as a nonequilibrium steady state) generated by external periodic perturbations. This is a concept of more recent origin. Third, stability in models when random (stochastic) perturbations are added, also a concept of relatively recent origin. From a methodological standpoint, these three types of stability fall into a natural sequence: simple deterministic stability, stability in the presence of deterministic periodic perturbations, and stability in the presence of random perturbations.

The study of static stability in Chapter III centers around the question of global stability in the Gause-Lotka-Volterra model. This 
latter model together with its associated concepts of a community matrix and an interaction matrix remains an active area of fleld and theoretical research in ecology (Seifert and Seifert 1976, Levins 1975). The model has been described as a useful empirical model (for example, by Culver 1975), in spite of its simple assumptions. Even the local stability behavior of this model was not clearly appreciated until a recent paper by Strobeck (1973), and most work using this model relies on local stability criteria together with a hope that global stability follows from local stability. Our analysis provides the most general results so far available on global stability in this model. Part of the significance of our results is that they provide retrospective justification for work which relied on local stability theory. More significantly, we obtain simple global stability criterla applicable to experimentally measured comunity matrices, and some interesting general criteria for global stability.

The concept of dynamic equilibrium under strong periodic perturbations originates with Stewart and Levin (1973) and was extended by Koch $(1974 a, b)$. We present in Chapter IV a detailed mathematical account of the nature of dynamic equilibrium and the conditions under which it occurs. We show that dynamic equilibrium states are a robust property of nonlinear population models. Our analysis suggests connections with the impact of grazing on vegetational diversity (Harper 1967, 1969), and the dynamics of lake ecosystems under perturbations (Parker 1974). The concept of a steady state driven by strong perturbations is emerging as a useful view of stability in studies of different ecological models which take spatial heterogeneity into account (Levin \& Paine 
1974). An important aspect of the work in Chapter IV is the development of dynamic equilibrium as a potentially useful concept in ecology. Finally, we study stochastic models in Chapter $V$. The inclusion of perturbations in ecological models is usually achieved by making the model stochastic. We consider specifically the addition of random nolse to nonlinear models. This problem has enfoyed constderable attention (May and MacArthur 1971, Lewontin \& Cohen 1969, Levins 1969, May 1974, Feldman \& Roughgarden 1975, Roughgarden 1975, 1976), and has generated mathematical controversy. It has also been the subject of attempts at an intuitive geometrical analysis (Leigh 1968, Gilpin 1974, Holling 1973). We apply powerful perturbation methods developed for physical problems (Papanicolau 1973, Ventsel and Friedlin 1970) and ecological problems (Ludwig 1976), to this problem and obtain unamb1guous qualitative results. These results provide the first general proof that stochastic stability is related to a deterministic Liapunov function. Our results are used to analyze certain previous geometrical assumptions concerning Liapunov surfaces, and to show that several of these assumptions are not true in general. The nonergodicity of many stochastic population models and the consequent mathematical and ecological implications are also discussed. Essentially nonergodicity in ecological models implies that the models will predict extinction of one or more populations with high probability over long times. In the light of empirical observations such extinctions may in fact occur in ecological communities considered in fsolation, and for such communities nonergodic models may be useful. However, the introduction of immigration and emigration processes stabilizes stochastic models and makes 
them ergodic, and such ergodic models may better approximate open ecosystems as opposed to isolated ones. We suggest that stochastic models may be used to explore the strategies which populattons adopt to minimize the probability of extinction. 
CHAPTER III

GLOBAI STABILITY IN

SIMPLE ECOLOGICAL MODELS

INTRODUCTION

The view that populations are effectively described by nonlinear dynamical models is widely supported by the ecological literature. However, it has been difficult to analyze in any general way the effect of nonlinearities on the behavior of dynamical models and hence in many applications the models are simply linearized. Unless one is willing to accept the idea that ecosystem dynamics are effectively linear (as Patten 1976 has suggested) the use of linearization generally excludes the effects of nonlinearities. In this chapter we are specifically concerned with the role of nonlinearities in determining the global stability properties of continuous nonlinear population models which have a single equilibrium point. Much work with models of the latter type has relied on a study of local stability properties determined by linearization around the equilibrium point, together with the assumption that local stability ensures global stability. Our purpose here is to present mathematical results on the relationship between local and global stability for a widely used class of nonlinear ecological models.

It is perhaps not generally appreciated that the global stability behavior of models can be of particular importance when the models are fitted to experimental data. Consider for example the Lotka-Volterra 
modeI (equation (3.8)) which describes community dynamics in terms of pairwise interactions between all species present. In the model the magnitude of the nonlinear terms becomes significant as soon as species populations deviate from equilibrium by some significant fraction of the population values at equilibrium. Therefore, in experiments where populations reach values far from equilibrium, the nonlinear or global dynamical behavior of the population model becomes very important. The global stability behavior of nonlinear models can be studied in terms of the behavior of suitable Liapunov functions, while local stability is determined by the eigenvalues of the matrix of coefficients obtained when the model is linearized around equilibrium. The approach we use here is to define a candidate Liapunov function for a class of models and then compare local stability criteria with criteria which ensure that the candidate function is in fact a Liapunov function.

We begin by defining a fairly general class of ecosystem models, and establish a criterion for the global stability of this class of models. We then show that an example of this general class of models is the Lotka-Volterra model, and present results on the relationship between local and global stability in the two-species and many-species Lotka-Volterra model. We show that in the two-species Lotka-Volterra model with self-regulation, local stability implies global stability and also a certain degree of structural stability. This is a long overdue result.

Our results on many-species Lotka-Volterra models describe the global stability behavior of models with locally qualitatively stable and D-stable community matrices. These last results have implications 
for the analysis of food web models (May 1974) and are of interest in the context of Levins' work (Levins 1974) on qualitatively speciffed models. Finally we consider the important question of whether relatively direct criteria for global stability can be found which are applicable to experimentally measured community matrices. We show that a rather simple sufficiency test for global stability of Lotka-Volterra models can indeed be found: the stability of the symmetric part of an experimentally measured community matrix will ensure local and global stability.

A summary of definitions and general theorems concerning mathematical stability in nonlinear models is presented in Appendix A. The mathematical ideas presented in Appendix A are used fairly extensively throughout this thesis.

\section{A GENERAL STABILITY THEOREM}

The sort of model we are concerned with describes an n-species ecosystem in terms of species biomasses $N_{i}, i=1, \ldots, n$, by a set of differential equations,

$$
\frac{1}{\mathrm{~N}_{i}} \frac{\mathrm{dN}_{\mathrm{i}}}{\mathrm{dt}}=\mathrm{F}_{i}(\overrightarrow{\mathrm{N}}), i=1, \ldots, \mathrm{n},
$$

where $\vec{N}=\left(N_{1}, \ldots, N_{n}\right)$. The model is assumed to have only one positive equilibrium point $\overrightarrow{\mathrm{N}}^{*}$ with each $\mathrm{N}_{i}^{*}>0$. Before defining the particular class of models to be studied in this section, we make a change of variables to a set $x_{1}, i=1, \ldots, n$, defined by

$$
N_{i}=N_{i}^{*} \exp \left(x_{i}\right)
$$

In the new variables the equations (3.1) become 


$$
\frac{d x_{i}}{d t}=F_{i}\left[N_{1} \exp \left(x_{1}\right), \ldots, N_{n} \exp \left(x_{n}\right)\right]=F_{i}(\vec{x})
$$

It is important to note that the stability behavior of (3.3) will be identical to the stability behavior of (3.1), and that any $x_{i}$ is a measure of the displacement of the biomass of species $i$ from its equilibrum value.

We are now in a position to describe the specific class of models which is to be studied. This class of models is analogous to the models described by Jeffreys (1975), and is motivated by the following notion. Consider a set of functions $f_{i}(z), i=1, \ldots, n$, which have the properties

$$
\begin{gathered}
z f_{i}(z)>0, z \neq 0, \\
f_{i}(0)=0 .
\end{gathered}
$$

Essentially the set $\vec{f}=\left(f_{1}\left(x_{1}\right), \ldots, f_{n}\left(x_{n}\right)\right)$ has the same sign structure as the set $\vec{x}$. In principle we could therefore measure biomass displacements from equilibrium in terms of the set $f_{i}\left(x_{i}\right), i=1, \ldots, n$, just as well as in terms of the $x_{i}$. An example of a set $f$ obeying (3.4) is the set $f_{i}(z)=\exp (z)-1$, all i.

The class of models to be studied is one where the per capita growth rates $F_{i}(\vec{N})$ or $F_{i}(\vec{x})$ can be written as a linear combination of an (arbitrary) set $f_{i}\left(x_{i}\right)$ defined by $(3.4)$. Such a class of models may be defined as the system of equations,

$$
\frac{d x_{i}}{d t}=F_{i}(\vec{x})=\sum_{j} b_{i j} f_{j}\left(x_{j}\right), i, j=1, \ldots, n .
$$

Here the $b_{i f}$ are a set of real numbers. The point $\vec{x}=\overrightarrow{0}$ is by assumption a unique equilibrium for the class of models (3.5). 
We now consider the global stability of models of the class (3.5) about the equilibrium point $\vec{x}=\overrightarrow{0}$. Let $B=\left(b_{i j}\right)$ be the matrix of the numbers $b_{i j}$ in (3.5), $B^{\prime}$ the transpose of $B$, and $d_{i}, i=1, \ldots, n$, a set of strictly positive numbers $\left(d_{i}>0\right.$, all $\left.i\right)$. Consider the function

$$
V(\vec{x})=\sum_{i} 2 d_{i} \int_{0}^{x_{i}} f_{i}(z) d z .
$$

In view of the properties of $f_{i}$ from $(3.4), V(\vec{x})>0$ for $\vec{x} \neq t$, and $V(\overrightarrow{0})=0$. Differentiating and using (3.5), we find

$$
\begin{aligned}
\frac{d V(\vec{x})}{d t} & =\sum_{i} \frac{\partial v}{\partial x_{i}} \frac{d x_{i}}{d t} \\
& =2 \sum_{i, j} f_{i}\left(x_{i}\right) d_{i} b_{i j} f_{j}\left(x_{j}\right) \\
& =\vec{f}^{\prime}\left(D B+B^{\prime} D\right) \vec{f},
\end{aligned}
$$

where $D$ is a matrix with only diagonal entries given by the $d_{i}, \overrightarrow{1}=$ $\left(f_{i}\left(x_{i}\right), \ldots, f_{n}\left(x_{n}\right)\right)$, and $\vec{f}$ is the transpose of $\vec{f}$. Now if the matrix $D$ is such that

$$
D B+B^{\prime} D=-C
$$

where $C$ is any symmetric positive definite matrix, then $\frac{d V}{d t}<0$ for $\vec{x} \neq \overrightarrow{0}, \frac{d V}{d t}=0$ for $\vec{x}=\overrightarrow{0}$.

Given (3.7), $V(\vec{x})$ is a Liapunov function which guarantees that the model (3.5) is globally asymptotically stable around the equilibrium $\mathrm{x}=$ 0 . Hence condition (3.7) is a sufficient condition for the global stability of models of the class (3.5). Since global stability ensures local stability, (3.7) is also a sufficient condition for the local stability of models of the class (3.5). We now show that the general stability condition obtained here is immediately applicable to a very popular nonlinear model. 


\section{A SPECIAL CASE}

It is easy to see that the widely used Lotka-Volterra model is an example of the class of models defined by (3.4) and (3.5). The generalized Lotka-Volterra model is

$$
\frac{1}{N_{i}} \frac{d N_{i}}{d t}=k_{i}+\sum_{j} \alpha_{i j} N_{j}=\sum_{j} \alpha_{i j}\left(N_{j}-N_{j}^{*}\right),
$$

where the $\alpha_{i j}$ are the elements of an interaction matrix $\alpha$. Using the transformation (3.2) to the variables $\vec{x}$, we rewrite $(3.8)$ as

$$
\frac{d x_{i}}{d t}=\sum_{j} \alpha_{i j} N_{j}^{*}\left[\exp \left(x_{j}\right)-1\right]=\sum_{j} a_{i j} f_{j}\left(x_{j}\right) .
$$

Here $a_{i j}=\left(\alpha_{i j} N_{j}^{*}\right)$ are the elements of a community matrix A. The functions $f_{j}(z)=\exp (z)-1, j=1, \ldots, n$, clearly satisfy the conditions (3.4). Therefore the Lotka-Volterra model (3.9) is just an example of (3.5), with $a_{i j}$ in (3.9) replacing $b_{i j}$ in (3.5).

A Lotka-Volterra model is characterized elther by the quantities $k_{i}, i=1, \ldots, n$, and the interaction matrix $\alpha$, or by the community matrix A. If we linearize (3.9) about the equilibrium point $\vec{x}=\overrightarrow{0}$, we get $\mathrm{d} \overrightarrow{\mathrm{x}} / \mathrm{dt}=\mathrm{A} \overrightarrow{\mathrm{x}}$. Therefore the local stability behavior of (3.9) is completely determined by the eigenvalues of $A$. The necessary and sufficient condition for the local stability of (3.9) is that, given an arbitrary symetric positive definite matrix $C_{1}$, there exists a symmetric positive definite matrix $G$ such that

$$
\mathrm{GA}+\mathrm{A}^{\prime} \mathrm{G}=-\mathrm{C}_{1} \text {. }
$$

Condition (3.10) is Liapunov's theorem (Gantmacher 1960). Out of the set of all possible community matrices A, condition (3.10) singles out 
those which yield locally stable dynamics.

Using the fact that (3.9) is an example of (3.5) we can apply the global stability condition (3.7). We then have a sufficient condition for the global stability of (3.9): The existence of a positive diagonal matrix $D$ such that

$$
n A+A^{\prime} D=-C
$$

where $\mathrm{C}$ is an arbitrary symmetric positive definite matrix. Comparing (3.10) and (3.11) we see that (3.11) is just a special case of (3.10). Let us refer to local stability and global stability for (3.9) about $\vec{x}=\overrightarrow{0}$, as LS and GS, respectively. Then if A satisfies (3.10), we have LS, which condition we write as $(3.10) \rightarrow$ LS. Conversely LS $\rightarrow(3.10)$. Also (3.11) $\rightarrow$ LS, (3.11) $\rightarrow$ GS. Hence (3.11) singles out of the set of all possible A those community matrices which yield locally stable as well as globally stable dynamics.

In those Lotka-Volterra models where the community matrix satisfies (3.11), we thus have LS $\rightarrow$ GS, local stability ensures global stability. When A does not satisfy (3.11), LS may or may not ensure GS. However the set of models for which A satisfies (3.11) are both interesting and useful, as we will now demonstrate.

\section{TWO-SPECIES LOTKA-VOLTERRA MODELS}

Consider the Lotka-Volterra model (3.8) with $i, j=1,2$, representing two-species interactions. We assume that both species are selfregulating so that $\alpha_{1 t}<0, i=1,2$. The other terms $\alpha_{12}, \alpha_{21}$ in the interaction matrix can take on values as follows:

$$
\alpha_{12}>0, \alpha_{21}>0:(+,+) \text {, }
$$




$$
\begin{gathered}
\alpha_{12}>0, \alpha_{21}=-\left|\alpha_{21}\right|<0 ;(+,-), \\
\alpha_{12}=-\left|\alpha_{12}\right|<0, \alpha_{21}=-\left|\alpha_{21}\right|<0 ;(-,-) .
\end{gathered}
$$

The ecological significance of these choices is discussed, for example, by Maynard Smith (1973). We now proceed to show that for all three cases in (3.12), the community matrices A satisfy ( 3.11$)$, and thus establish global stability properties for two-species interactions. We suppose an equilibrium point $\left(\mathrm{N}_{1}^{*}>0, \mathrm{~N}_{2}^{*}>0\right)$ exists. The community matrix for all cases in (3.12) is

$$
A=\left(\begin{array}{ll}
\alpha_{11} \mathrm{~N}_{1}^{*} & \alpha_{12} \mathrm{~N}_{2}^{*} \\
\alpha_{21} \mathrm{~N}_{1}^{*} & \alpha_{22} \mathrm{~N}_{2}^{*}
\end{array}\right),
$$

and the local stability criterion (from (3.10) or the equivalent RouthHurwitz theorem, Gantmacher (1960)) for all cases in (3.12) is

$$
\alpha_{11} \alpha_{22}>\alpha_{12} \alpha_{21}
$$

To show that $A$ in (3.13) satisfies (3.11), we need only write down suitable numbers $d_{i}, i=1,2$, so that ( 3.11 ) holds. For convenience we will write $d_{1}, d_{2}$ and the matrix $-C=D A+A^{\prime} D$. In the three cases (3.12a) through $(3.12 c)$ we choose

$$
\begin{aligned}
& \mathrm{d}_{1}=\frac{1}{2\left|\alpha_{12}\right| \mathrm{N}_{2}^{*}}, \mathrm{~d}_{2}=\frac{1}{2\left|\alpha_{21}\right| \mathrm{N}_{1}^{*}}, \\
& -\mathrm{C}_{11}=\frac{\alpha_{11} \mathrm{~N}_{1}^{*}}{\left|\alpha_{12}\right| \mathrm{N}_{2}^{*}}=\left(\mathrm{DA}+\mathrm{A}^{\prime} \mathrm{D}\right)_{11}, \\
& -\mathrm{C}_{22}=\frac{\alpha_{22} \mathrm{~N}_{2}^{*}}{\left|\alpha_{21}\right| \mathrm{N}_{1}^{*}}=\left(\mathrm{DA}+\mathrm{A}^{\prime} \mathrm{D}\right)_{22}, \\
& -\mathrm{C}_{12}=-\mathrm{C}_{21}=1,
\end{aligned}
$$




$$
\begin{aligned}
& -c_{12}=-c_{21}=0, \\
& -c_{12}=-c_{21}=-1 .
\end{aligned}
$$

For both the $(+,+)$ and $(-,-)$ interactions the local stability criterion (3.14) guarantees that $C$ in (3.15a) and (3.15c) is positive definite and so guarantees global stability. For the $(+,-)$ interaction it is seen from (3.15b) that the assumptions on $\alpha$ and the existence of an equilibrium point are sufficient to ensure global stability.

The case $(3.12 \mathrm{~b})$ with $(+,-)$ interaction has been previously examined by Walter (1973), who established the same result. From a mathematical standpoint it is worth pointing out, in passing, a connection between Walter's proof and ours. Our analysis is based on the Liapunov function (3.6), which for the two-species Lotka-Volterra model becomes, using (3.9)

$$
v(\vec{x})=2 d_{1}\left[\exp \left(x_{1}\right)-x_{1}-1\right]+2 d_{2}\left[\exp \left(x_{2}\right)-x_{2}-1\right]
$$

The Liapunov function used by Walter is just $W(\vec{x})=\exp [V(\vec{x})]$. This nonlinear relationship between two Liapunov functions for the same model serves to emphasize the non-uniqueness of Liapunov functions which contain the same stability information.

The results we have established for two-species Lotka-Volterra models justify the use of local stability analysis alone in working with such models. Additionally, given that any one of the two-species models specified by (3.12) is locally and so globally stable, it follows that the mode1 is also structurally stable (De Baggis 1955). It is important to stress that structural stability means stability against small perturbations of the right-hand sides of the equations (3.8). It is clear 
from the local stability criterion that one can perturb the parameters $\alpha_{i j}$ sufficiently to destroy the inequality (3.14), at which point structural instability occurs. However, in the space of the four parameters $\alpha_{i j}$, if we are within the region defined by (3.14) the two-species model will be structurally stable. To illustrate why structural stability can be useful, consider a two-species Lotka-Volterra model with $(-,-)$ interactions as in (3.12c). The isoclines $\mathrm{dN}_{1} / \mathrm{dt}=0, \mathrm{dN}_{2} / \mathrm{dt}=0$ for this model are straight lines in $\left(\mathrm{N}_{1}, \mathrm{~N}_{2}\right)$ space. If we introduce some degree of curvature into these isoclines, say by adding a term of the form (constant) $\mathrm{N}_{1} \mathrm{~N}_{2}$, to the right-hand side of the model (3.8), then the structural stability property ensures that the qualitative behavior of the perturbed model is the same as of the original model. This last fact is nicely illustrated by Maynard Smith's (1974) discussion of precisely such a perturbed model.

This section illustrates the usefulness of our general stability condition (3.7) when applied to two-species interactions. We now go on to obtain a number of results on the global stability of many-species interactions.

\section{MANY-SPECIES MODELS}

The general global stability condition (3.7) can in practice be used in two ways. Given a particular model of class (3.5) one can arbitrarily select some suitable matrix $C$, and try to obtain $D$ as a solution to the matrix equation (3.7). Alternatively one can first specify $D$ to be a particular matrix and then test $\left(D B+B^{\prime} D\right)$ to see if it is symmetric negative definite. This second approach is fruitful when one 
discusses special examples of $B$, and will be adopted in parts of this section. We now present results on global stability in models of manyspecies interactions. Our results will be stated in terms of the Lotka-Volterra model (3.8), (3.9), but most of them apply to the general class of models ( 3.5$)$. The results may be translated to model (3.5) simply by replacing matrix A everywhere by the matrix $B$, except in cases where $A$ is analyzed as the product of $\alpha \mathbb{N}^{*}$.

\section{D-Stability}

Consider an n-species Lotka-Volterra model (3.9) with a community matrix A. Assume that A satisfies the global stability condition (3.11). Equation (3.11) also happens to be a sufficient condition for A to have the property of D-stability, i.e., if A satisfies (3.11), then the matrix PA will be stable (real parts of eigenvalues of PA will be negative) where $\mathrm{P}$ is any matrix with strictly positive diagonal elements and all other elements zero (Ouirk and Ruppert 1965). Therefore condition (3.11) identifies a subset of community matrices A which yield locally and globally stable dynamics, and are also D-stable. Recall that the elements of $A$ are $a_{1 j}=\alpha_{i j} N_{j}^{*}$, and so we can write $A=\alpha N^{*}$ where $\alpha$ is the interaction matrix and matrix $N^{*}$ has only diagonal entries given by the $\mathrm{N}_{i}^{*}$. We can see right away that if A satisfies (3.11) then $A$ is $D$-stable and hence $\alpha$ is also stable. Additionally if $A=\alpha N^{*}$ satisfies (3.11), then the matrix $\alpha M^{*}$ is also stable, where $M^{*}$ has only positive diagonal entries. This last conclusion leads to an interesting geometrical interpretation of the D-stability property.

Consider the equations $\mathrm{dN}_{i} / \mathrm{dt}=0, i=1, \ldots, \mathrm{n}$. In the $\mathrm{n}$-dimensional space of $\vec{N}$, these equations define hyperplanes whose interaction 
is at $\vec{N}^{*}$. Now imagine that the hyperplanes are translated so that their intersection is now at $\vec{M}^{*}=\left(m_{1}, m_{2}, \ldots, m_{n}\right)$. We could achieve this by changing $k_{i}, i=1, \ldots, n$, in (3.8) while keeping $\alpha$ fixed. The new hyperplanes define a Lotka-Volterra model with the same interaction matrix but a new community matrix $\alpha M^{*}$, where $M^{*}$ has only diagonal entries $\left(m_{1}, \ldots, m_{n}\right)$. If the original $A=\alpha N^{*}$ satisfies (3.11), then $\alpha M^{*}$ is also stabie by the D-stability property. Therefore D-stability of A means that the stability of the model is unaffected by altering the location of the equilibrium point in $\overrightarrow{\mathrm{N}}$-space.

\section{Qualitative Stability}

In studying many-species interactions, it is very often true that available data provide only qualitative information about the signs of the interaction matrix elements. It is then useful to study the stability properties of models in which only the signs of the elements $a_{i j}$ (or $\alpha_{i j}$ ) are known. We first point out that since all $\mathrm{N}_{i}^{*}>0$, the matrix A has the same sign structure as the matrix $\alpha$, and so we can legitimately work with either matrix.

Any given natrix is sald to be qualitatively stable (or sign stable) if it is stable irrespective of the magnitudes of its elements. Conditions for sign-stability are discussed by Ouirk and Ruppert (1965). We consider the community matrix $A$ of an n-species Lotka-Volterra model, and assume that A satisfies the global stability condition ( 3.11$)$. It happens that (3.11) is also the necessary and sufficient condition for A to be a qualitatively stable matrix with strictly negative diagonal elements.

Therefore if the community matrix A of a given Lotka-Volterra 
model, (i) has strictly negative diagonal elements $\left(a_{i i}>0, a 11 i\right)$, and (ii) is qualitatively stable, then the model will be locally as well as globally stable. This result and the preceding result on D-stability are interesting from a mathematical standpoint. It has been felt (for example, by Siljak 1975) that qualitative stability and D-stability are useful only in analyzing local stability properties of models. However they turn out to be relevant to the globally stable dynamics of not only the Lotka-Volterra model, but also models of the general class (3.5).

\section{SUFFICIENCY CONDITIONS: \\ THE SYMMETRIC-PART TEST}

In the particular context of the Lotka-Volterra models, the condition (3.11) is the most general condition for global stability so far avaisable. We illustrate this by considering two well known special cases, and demonstrating local and global stability.

First, let $\alpha$ be symmetric negative definite. This case was used by MacArthur (1970) in his elegant study of species packing. We make the choice $D=N * / 2$ so that conditicn (3.11) becomes $N * \alpha N^{*}=-C$, which is clearly true. Thus (3.11) holds, ensuring local and global stability in this case.

Second, let $\alpha$ be antisymetric. Again we choose $D=N * / 2$ and find $D A+A^{\prime} D=0$, which means that the Liapunov function (3.6) obeys $d V / d t=$ 0 , the usual result. Alternatively if $\alpha$ is antisymmetric only in its off-diagonal entries, and has a negative diagonal $\alpha_{i i}<0$, all $i$, then $D=N * / 2$ yields $D A+A^{\prime} D=N^{*} \hat{\alpha} N^{*}$ where $\hat{\alpha}$ has only diagonal elements $\alpha_{i 1}$ and zeros elsewhere. This last equation shows that ( 3.11 ) is satisfied and local and global stability follow. 
Finally we will set down one specific sufficient condition for local and global stability of the Lotka-Volterra model. This condition may be a useful one in the context of experimental work on community stability in which the community matrix A is actually computed from experimental data. The usual procedure is to assemble $A$ and then test it for stability, or in other words to test for local stability. If we consider (3.11) and set $D=I$, the unit matrix, we obtain the following simple sufflcient condition for global stability,

$$
A+A^{\prime}=-C \text {. }
$$

The significance of (3.17) lies in the fact that if the symmetric-part of a community matrix $A$ is stable then the model is locally and globally stable. Given an experimentally measured community matrix A it would therefore be straightforward and quite possibly profitable to test the symmetric part of A for stability.

\section{DISCUSSION}

The general stability theorem (3.5) provides a number of interesting results which illustrate the richness in the dynamical properties of nonlinear ecological models. The analysis of qualitative stability shows that simple food web models (May 1974) can have globally stable dynamics, and emphasizes the role of self-regulation in stabilizing many-species systems. The symmetric-part test of the preceding section is an example of a potentially useful, simple test for global stability. It seems clear that the study of the global, as opposed to local, stability oroperties of nonlinear models can provide a great deal of useful information about the behavior of models in relation to reality. 
CHAPTER IV

\section{DYNAMIC EQUILIBRIUM IN SIMPLE ECOLOGICAL MODELS}

\section{INTRODUCTION}

Systems of nonlinear differential equations are widely used to model simple ecosystems. Typically the models describe the ecosystem in terms of the biomasses or populations of the interacting species. Two questions are then examined: the existence of time-independent equilibrium values of the biomasses, and the stability of this equilibrium against perturbations (May 1974, Maynard Smith 1974). We call this type of endogenous equilibrium a static equilibrium. Static equilibrium analysis provides conditions for stable coexistence in model ecosystems. In this chapter, we examine an idealized case of dynamic equilibrium in simple model ecosystems. This dynamic equilibrium is exogenously maintained by a periodic reduction in the biomasses of the interacting systems. We find that the occurrence of a dynamic equilibrium depends upon topological features of the solutions to the equations of the model. Coexistence in a dynamic equilibrium occurs for a rather wide range of conditions as compared with coexistence in a static equilibrium. The perturbations that maintain a dynamic equilibrium, periodic reductions in biomass, were first considered by Stewart \& Levin (1973) as a paradigm for a 'seasonal' mode of coexistence. More recently, Koch (1974) has carried out numerical studies of the effect of such perturbations. We cover somewhat similar ground from an analytical standpoint, 
and examine the general mathematical features that make dynamic equilibrium possible. It seems likely that such equilibrium states are a rather general feature of simple models, and that dynamic equilibrium may be a useful 'strategic' notion in ecological theory.

We consider two interacting species with biomasses $N_{i}(t), i=1,2$, at time $t$. The general model used is

$$
\frac{d N_{i}}{d t}=N_{i} F_{i}(\vec{N}),
$$

where $\vec{N}=\left(\mathrm{N}_{1}, \mathrm{~N}_{2}\right)$. The biomasses are determined by specifying their initial values at some initial time $t=0$. The perturbations we consider are periodic reductions in the biomasses $\vec{N}$. We choose a period $T$ and a 'kill factor' or 'dilution factor' $k$. Then the perturbation consists of a $k$ - fold reduction in the biomasses at the end of every $T$ units of elapsed time. A special form of periodicity is thus imposed on the dynamics of the system (4.1) by this perturbation. If we choose a suitable set of parameters specifying the form of the equations (4.1), and some general initial biomass $\vec{N}(0)$ and apply this perturbation, we find that over the course of a few periods the biomasses begin to repeat a particular sequence of values from each period of length $T$ to the next. This final sequence of values of $\vec{N}$ is the dynamic equilibrium state. For an appropriate set of model parameters a unique dynamic equilibrium state exists for each suitable choice of $\mathrm{T}$ and $k$.

The mathematical features of the progress towards a dynamic equilibrium can be clearly understood by studying the solutions of the system (4.1). These solutions can be represented as trajectories in a phase-plane which has $\left(\mathrm{N}_{1}, \mathrm{~N}_{2}\right)$ as coordinates. We devote the next section to an examination of these phase-plane trajectories; in particular 
we consider the convergence of these trajectories. The particular case of the system (4.1) that we use is the Lotka-Volterra form with selfdamping. Despite its many inadequacies this model remains useful as a first order example of a nonlinear system, and strong results based on the full nonlinear (not linearized) Lotka-Volterra system are of ten good indications that these results may carry over to more general systems. The results on the convergence of trajectories enable us to discuss the achievement of dynamic equilibrium in the Lotka-Volterra system and the various ranges of parameters that allow coexistence in dynamic equilibrium, following which we consider more general models. Finally, the last section discusses the possible 'strategic' and other aspects of this type of dynamic equilibrium in ecological theory.

\section{CONVERGENCE OF TRAJECTORIES}

This section is a prelude to the discussion of the processes that generate dynamical equilibrium under periodic perturbations. The question we study here is the behavior of phase-plane trajectories of systems of type (4.1) which start from different initial points in the plane. We establish conditions under which these trajectorles converge for the case of the Lotka-Volterra system. In the next section we show how this convergence drives the system into a dynamic equilibrium state. We write the Lotka-Volterra equations in the form (Gause \& Witt 1935)

$$
\frac{d N_{i}}{d t}=\frac{r_{i} N_{i}}{K_{i}}\left(K_{i}-N_{i}-a_{i} N_{j}\right), \quad i \neq j, i, j=1,2,
$$

where the $r_{i}, k_{i}$ are 'intrinsic' growth rates and carrying capacities, 
respectively, and the $a_{i}$ are interspecies interaction coefficients. A particular solution of (4.2) is determined by specifying initial values $\vec{N}(0)$ and can be represented as a curve or trajectory in the phase-plane. Formally we can say that specifying a particular set of initial values completely specifies the trajectory that goes through that point on the phase-plane.

The question we address ourselves to is the relative behavior of two trajectories that start from different initial points in the phaseplane. Let these initial points be $\vec{N}(0)$ and $\vec{N}(0)+\vec{y}(0)$ respectively, with corresponding solutions $\vec{N}(t)$ and $\vec{N}(t)+\vec{y}(t)$. We wish to examine the behavior of the distance $|\vec{y}(t)|$ between the two trajectories as a function of time. The algebra becomes simpler if we make a change of variables at this point. Since $N_{i}>0$, we introduce variables $z_{1}=1 \mathrm{n}$ $\left(N_{i}\right)$. The two trajectories we are considering may now be equivalently specifled by initial points $\vec{z}(0)$ and $\vec{z}(0)+\vec{x}(0)$, with points on the trajectories being $\vec{z}(t)$ and $\vec{z}(t)+\vec{x}(t)$, respectively. The distance between trajectories can be described by $|\vec{x}(t)|$, and we examine the behavior of this quantity in time.

Using the change of variables to the $z_{i}$, and the equations (4.2), we obtain equations for the rates of change of the $x_{i}(t)$ with time:

$$
\frac{d x_{i}}{d t}=-\frac{r_{i}}{k_{i}}\left\{N_{i}\left[\exp \left(x_{i}\right)-1\right]+a_{i} N_{j}\left[\exp \left(x_{j}\right)-1\right]\right\} \text {. }
$$

In this equation the $\vec{N}(t)$ are functions of time that are in principle completely determined by the fact that they are solutions of (4.2) with particular initial values $\vec{N}(0)$. Therefore we can look upon the functions $N_{i}(t)$ in $(4.3)$ as time-dependent coefficients. Observe that the 
point $\vec{x}^{*}=(0,0)$ is an equilibrium point of the system $(4.3)$. We can now study the behavior of $|\vec{x}(t)|$, i.e., the distance between trajectories, at time $t$ in terms of the properties of the equations (4.3).

Our interest in $x(t)$ now centers on one question: does $|\vec{x}(t)| \rightarrow 0$ as $t \rightarrow \infty$ ? If and when this happens, trajectories which start from initial points separated by some distance $|\vec{x}(0)|$ will converge in time. In terms of the equations (4.3) the question becomes: is the point $x^{*}$ a stable equilibrium point for the system (4.3)? To answer this question, we turn to the apparatus of Liapunov theory (Hahn 1967).

We introduce the function

$$
V(\vec{x})=\sum_{i}\left[\exp \left(x_{i}\right)-x_{i}-1\right]
$$

which is positive-definite, continuously differentiable, and such that $|V(\vec{x})| \rightarrow \infty$ when $|\vec{x}|+\infty$. Using (4.3) we find that the derivative of this function is

$$
\frac{d V}{d t}=\underset{i, j}{\sum}\left[\exp \left(x_{i}\right)-1\right] b_{i j}\left[\exp \left(x_{j}\right)-1\right]
$$

where the quantities $b_{i j}$ form the elements of a matrix $B$ and are

$$
b_{i 1}=-\frac{r_{i} N_{i}}{k_{i}}, b_{i j}=-\frac{r_{i} N_{j}^{a}}{k_{i}}, i \neq j,
$$

Clearly $\mathrm{dV} / \mathrm{dt}$ is negative-definite if the matrix $\mathrm{B}$ is negative-definite. The necessary condition for this to happen is

$$
a_{1} a_{2}<1
$$

Only if condition $(4.7)$ holds will $V(\vec{x})$ and $d V / d t$ satisfy all the conditions that make $V(\vec{x})$ a Liapunov function for system (4.3) around the equilibrium $\vec{x}^{*}$. This means that under condition (4.7) the distance 
$|\vec{x}(t)| \rightarrow 0$ with time, and so the trajectories separated by any initial distance $|\vec{x}(0)|$ will converge. We will refer to condition (4.7) as the condition for convergence for the Lotka-Volterra system.

EQUILIBRIUM UNDER PERIODIC PERTURBATIONS

In this section we show that under the condition for convergence (4.7) the Lotka-Volterra system (4.2) displays dynamic equiltbrium states under periodic perturbations. The perturbations are characterized by a period $\mathrm{T}$ and $\mathrm{a}$ biomass reduction factor $\mathrm{k}$.

Consider two trajectorles of the Lotka-Volterra system which start from initial points $\vec{N}(0)$ and $\vec{M}(0)$ respectively, so that the inftial separation between trajectories is $\mathrm{d}(0)=|\vec{N}(0)-\vec{M}(0)|$. We assume that conditicn (4.7) holds. In a time $T$ these trajectories will be at final points $\vec{N}(T)$ and $\vec{R}(T)$ as determined by solving the equations $(4.2)$, and the separation between trajectories will be $d(T)=|\vec{N}(T)-\vec{M}(T)|$. Since the condition for convergence is aso:umed, the distance between trajectories decreases in time, i.e., we uㅡㄴㅡ have $d(T)<d(0)$.

Let us now apply the perturbation. Ihis requires that at time $T$ we reduce the biomasses by the factor $k$. Thus for the two trajectories above we divide the final biomasses by $k$, and obtain new inftial points $\vec{N}^{\prime}(0)=\vec{N}(T) / k$ and $\vec{M}^{\prime}(0)=\vec{M}(T) / k$. Using these initial points in $(4.2)$ we generate solutions which after another period of time $T$ reach new final points $\vec{N}^{\prime}(T)$ and $\vec{M}^{\prime}(T)$ in the phase-plane. The perturbation is now applied again, and the process is iterated indefinitely. For the argument here, we concentrate on the first iteration step which we have detailed above. The new initial points obtained after a first 
application of the kill factor $k$ are separated by a distance $d^{\prime}(0)=$ $\left|\vec{N}^{\prime}(0)-\vec{M}^{\prime}(0)\right|=|(\vec{N}(T) / k)-(\vec{M}(T) / k)|=d(T) / k$. Recalling that the condition for convergence holds, and if $k>1$, we see that $d^{\prime}(0)<d(T)<d(0)$. The final points at the end of the first iteration are separated by a distance $d^{\prime}(T)=\left|\vec{N}^{\prime}(T)-\vec{M}^{\prime}(T)\right|$ and because condition (4.7) holds we must have $d^{\prime}(T)<d^{\prime}(0)$. Summarizing the relationships between these separations, we have the following: the inftial distance between two trajectories is $d(0)$, the separation at end of the first period is $d(T)$, the initial separation for the first iteration step is $d^{\prime}(0)$, the separation at end of the first iteration step is $d^{\prime}(T)$ and

$$
d^{\prime}(0)<d(0) ; d^{\prime}(T)<d(T) \text {. }
$$

These inequalities show that the process of applying the perturbation and carrying out the first iteration step acts as a contraction of the separation between trajectories in the phase-plane. Formally let us define an operator $\Gamma$ which acts on any point $\vec{N}$ in the phase-plane and produces a new point $\vec{N}^{\prime}=\Gamma(\vec{N})$ through the following sequence of operations: using $\vec{N}(0)$ as an initial point for equations (4.2) obtain a point $\vec{N}(T)$ by solving the equations over a period $T$, then obtain $\vec{N}^{\prime}$ as $\vec{N}(T) / k$. The inequalities we have for two trajectories with initial points $\mathrm{N}$ and $\mathrm{M}$ in the phase-plane can then be formally rewritten as $|\Gamma(\vec{N})-\Gamma(\vec{M})|<|\vec{N}-\vec{M}|$. Thus $\Gamma$ is a contraction mapping. This property is illustrated schematically in Figure 1.

Consider now a trajectory starting from some general initial point $\overrightarrow{\mathrm{N}}(0)$. We apply the perturbation iteratively, which means that we successively obtain new initial points $\Gamma(\vec{N}(0)), \Gamma^{2}(\vec{N}(0)), \Gamma^{3}(\vec{N}(0))$, at the end of the first, second, third iterations, respectively, and so on. 


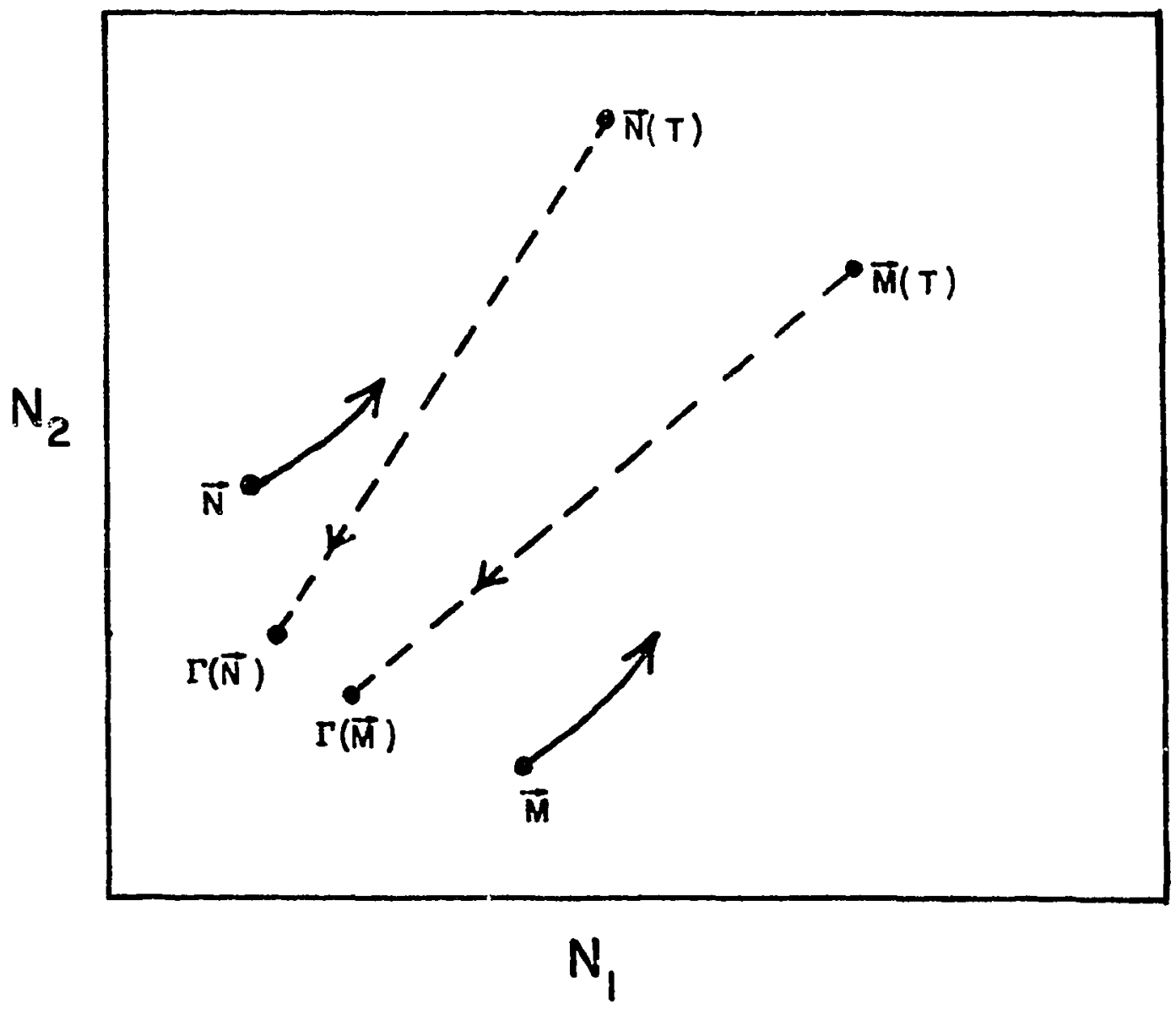

Figure 1. Representation of the action of the contraction mapping $\Gamma$. The points $\vec{N}, \vec{M}$ are taken as initial points for the model equation, and points $\overrightarrow{\mathrm{N}}(\mathrm{T}), \overrightarrow{\mathrm{M}}(\mathrm{T})$ are obtained by moving along phase-plane trajectories (solid lines). The kill factor then translates these new points into the final points $\Gamma(\vec{N})$, $\Gamma(M)$. Dashed lines show the effect of the kill factor. 
The fact that $\Gamma$ is a contraction mapping means that this sequence converges towards a unique point. This unique point, which we can call $\overrightarrow{\mathrm{N}}^{*}$, is defined by the property that $\Gamma\left(\vec{N}^{*}\right)=\vec{N}^{*}$. The trajectory obtained by solving equations (4.2) with $\overrightarrow{\mathrm{N}}^{*}$ as an initial point over a period of $\mathrm{T}$ units will have a final point $\vec{N}^{*}(T)=k \vec{N}^{*}$. This trajectory is our dynamic equilibrium state.

The achievement of a dynamic equilibrium therefore depends on two conditions: first, the condition for convergence must hold, and second, we must have a kill factor $k>1$. Furthermore the particular dynamic equilibrium state that is achieved, i.e., the particular point $\overrightarrow{\mathbb{N}}^{*}$ that is the initial point for the equilibrium trajectory, depends upon the parameters in the model and the choice of $T$ and $k$. Once these quantities are given definite values, there is a unique equilibrium state toward which the system evolves independent cf which initial point we first start out from.

The arguments presented thus far provide an analytical view of the processes that generate a dynamical equilibrium under periodic perturbations. From equation (4.3) of the previous section we see that the nonlinear terms in the original equations (4.2) are responsible for driving the convergence of phase-plane trajectories. This convergence is a necessary condition for dynamic equilibrium to be achieved. The type of dynamical equilibrium state that we discuss here is similar to the stroboscopic limit cycles that are observed in other periodically perturbed systems (Minorsky 1962).

Numerical calculations can be done in a straightforward way to demonstrate the appearance of a dynamic equilibrium state. Several 
examples are presented by Koch (1974), where plots of the biomasses of both species as functions of time are shown over several successive steps of the iteration. The limitations on numerical accuracy inherent in computer calculations result in the apparent achievement of a specific equilibrium state within a very few iteration steps. The rapid progress to equilibrium that is typically observed does however suggest that the contraction mapping that drives the system to equilibrium can be very 'strongly contracting'. Koch points out that he was unable to obtain dynamical equilibrium states numerically for the Lotka-Volterra model when $a_{1}=a_{2}=1$, and when $a_{1} a_{2}=1$. This clearly follows from our condition (4.7).

There is a rather transparent way of looking at the results of a numerical calculation of the dynamic equilibrium state. Consider any one step in the iteration, say the $j$ th step. Let the biomasses of the two species at the beginning and end of this iteration step be $N_{1}^{j}(0)$ and $\mathrm{N}_{i}^{j}(T)$, respectively, where $i=1,2$, and the superscript $j$ refers to the iteration step. We define the ratios of end-of-step biomass to beginning-of-step biomass as $R_{i j}=\left(N_{i}^{j}(T)\right) /\left(N_{i}^{j}(0)\right)$. From the calculation we obtain and plot these ratios as a function of the iteration step number j. Recalling that the dynamical equilibrium state is defined by having a ratio of $k$ between final and initial biomasses, we look for the convergence of both ratios, $R_{i j}+k$. An example of this is shown in Figure 2 . The number of iteration steps taken to achieve near-equilibrium is a measure of the speed at which equilibrium is reached. This speed or rate depends on the choice of the parameters $T$ and $k$. We now discuss the effect of different choices of these parameters. As we pointed out 


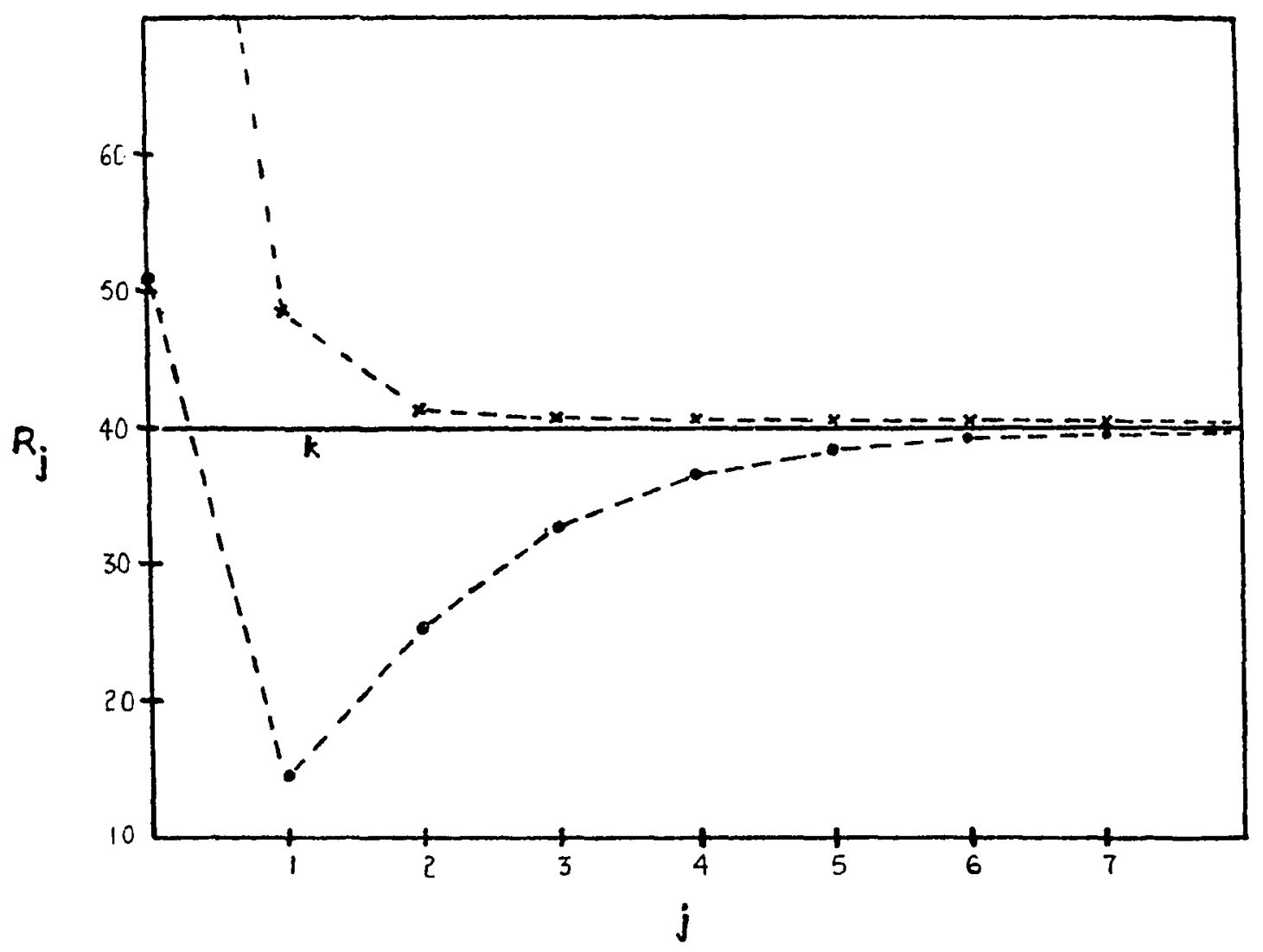

Figure 2. Biomass ratios for successive iteration steps using the Lotka-Volterra model. Dots and crosses indicate ratios for the two species. The solid line indicates the kill factor. Here $k=40$. The value of $r_{1}$ is chosen so that with unrestricted growth species 1 doubles every time unit. The period of the perturbation $T$ is 10 time units; $a_{1} a_{2}=0.6 ; \mathrm{K}_{1} / \mathrm{K}_{2}=1 ; \mathrm{r}_{2} / \mathrm{r}_{1}=2$.
Initial biomasses are chosen equal. 
earlier, the convergence of trajectories is driven by the nonlinear terms in the equations (4.2). The nonlinear terms dominate the equation for large values of the biomass, and we expect trajectories to converge more rapidly than when the biomasses are low. For low biomass values, we expect that the rates of change of the biomasses are roughly exponential with growth rates $r_{i}$. We define a quantity $r$ either as $\left(r_{1} r_{2}\right)^{\frac{1}{2}}$ if the two $r_{i}$ are similar in magnitude, or, if not, as the smaller of the two. Then $\mathrm{r}^{-1}$ defines a time scale over which we expect roughly exponential growth at low biomass values. Our choice of a range for $T$ should be such that the effect of the nonlinear terms be significant, so we can qualitatively say that $r T \gg 1$ is a suitable approximate criterion for rapid approach to equilibrium. We can now consider the kill factor $k$.

We have stated earlier that we need $k>1$. In practice $k$ has an approximate upper limit. Again this is related to the importance of nonlinear terms in the equations for $N$. If $k$ is made too large, one or both of the biomasses may be driven to values so low that the nonlinear terms remain small over the entire period $T$ in each iteration step. The convergence of trajectories during each step is then too weak to drive the system towards equilibrium. Very roughly we may set exp(rT) as an upper limit on $k$, with actual values of $k$ ranging over a few orders of magnitude below. In numerical work it usually turns out that this is not a significant limitation. The qualitative criteria of the last two paragraphs are related to the question of necessary and sufficient conditions for convergence of trajectories. The condition (4.7) is strictly only a necessary condition for convergence. The necessary and 
sufficient condition involves values of $\vec{N}(t)$ along the trajectories.

Our discussion thus far has focused on dynamic equilibrtum in the context of the Lotka-Volterra model. Three additional points need to be made before we go on to examine more realistic models in the next section. The first point is that the preceding analysis can formally be extended to cover many-species interactions (see Appendix A). It could also be used for three types of two-species interactions. Depending on the signs of the interaction coefficients $a_{i}$, these types are competition, predator-prey, and mutualism or symbiosis. From the point of view of realism as a criterion for using Lotka-Volterra models, we feel that they are best used to study competitive interactions. The second point concerns a comparison for the case of competitive interactions between our condition (4.7) for dynamical equilibrium and the conditions (Gause \& Witt 1935) for stable static equilibrium. The comparison is facilitated by plotting the two conditions in $\left(a_{1}, a_{2}\right)$ parameter space. The static equilibrium conditions are

$$
a_{i}<\frac{k_{i}}{k_{j}}, \quad a_{1} a_{2}<1 .
$$

The plot is shown in Figure 3. We see that dynamic equilibrium can occur over extended regions of parameter space which are inaccessible to systems in a stable static equilibrium. In biological terms, it appears that coexistence in dynamical equilibrium can extend to pairs of species with very unequal competitive ability.

The third and last point is that under periodic perturbations, models such as (4.2) may display complex dynamical behavior other than dynamical equilibrium states. Recall that the convergence of 


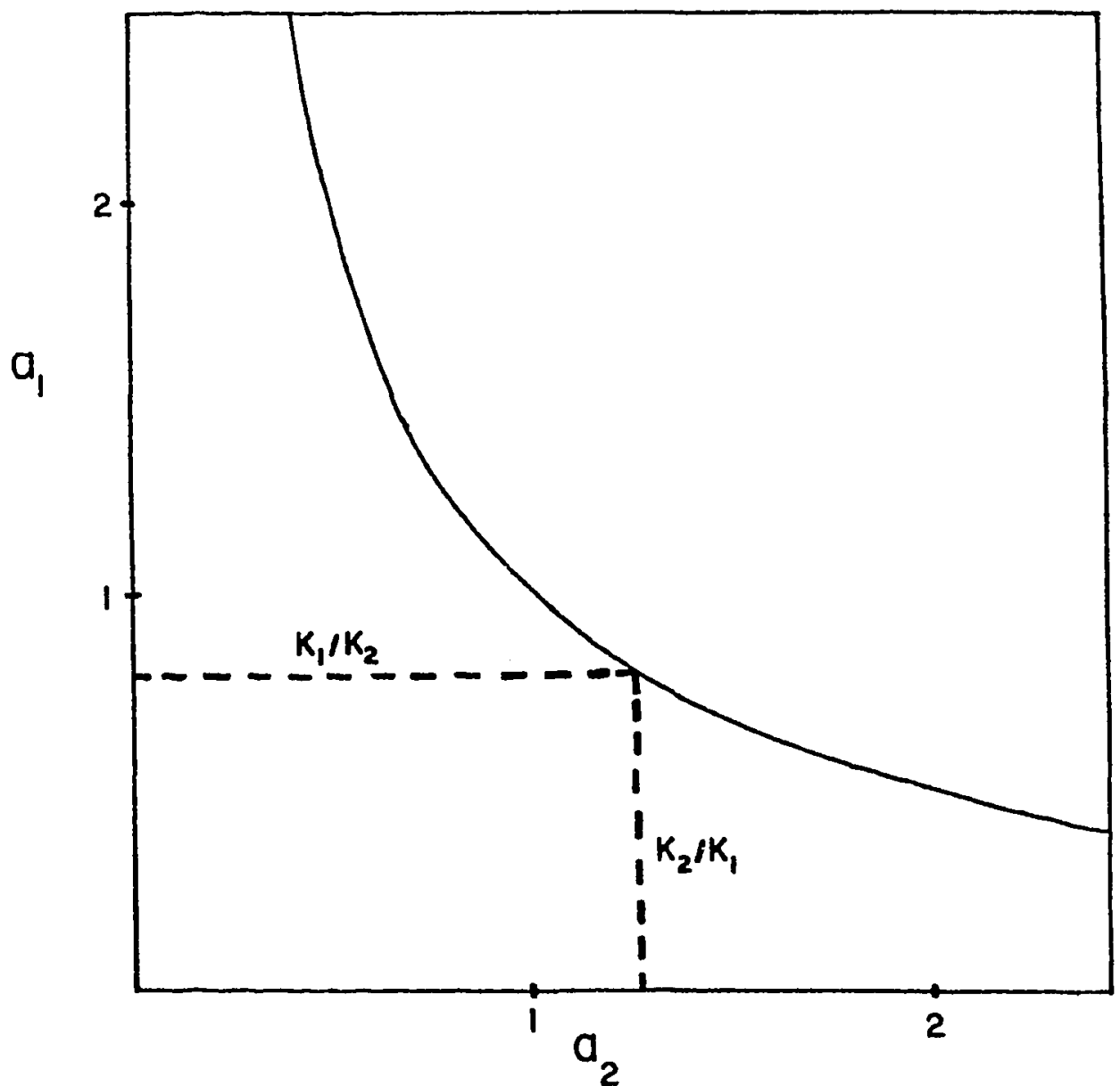

Figure 3. Plot of parameter space for the Lotka-Volterra mode1 of two-species competition. For coexistence at a static equilibrium point, the accessible region of parameter space is the rectangle enclosed by the axes and the dashed lines. Coexistence in a dynamic equilibrium state makes the entire region under the solid curve $\left(a_{1} a_{2}=1\right)$ accessible. 
trajectories was studied in earlier sections in terms of the separation $\vec{x}(t)$ of two trajectories. In order to obtain dynamical equilibrium state it is necessary that $\vec{x}^{*}=(0,0)$ be an asymptotically stable equilibrium point for the system (4.3) describing $\vec{x}(t)$. Now consider the possibility that the separation $\vec{x}(t)$ described by (4.3) displays stable limit-cycle behavior. In such a case $|\vec{x}(t)|$ does not go to zero in time, but instead varies periodically over some range of values. Qualitative1y this means that there will exist a band of trajectories around any initial trajectory in $\vec{N}$-space, and that after several periods of the perturbation the trajectory in $\vec{N}$-space will be within this band. This is not all: given a limit cycle for $\vec{x}(t)$ around $\vec{x}^{*}$, a new time scale emerges, which is the period $\tau$ of the limit cycle. Now if the period of the perturbation $T$ exceeds $\tau$, the biomass trajectories in any two succeeding periods of the perturbation will have a separation that varies slgnificantly over a time interval of $T$. If $T=\tau$, then in any two succeeding periods of the perturbation the biomass trajectories will have a separation that changes cyclically over a time interval of $T$. This last phenomenon might be called entrainment of periods. In our subsequent discussion we restrict ourselves to the situation where $\vec{x}^{*}$ i: a stable equilibrium for (4.3).

DYNAIIIC EQUILIBRIUM IN GENERAL MODELS

Theory

Our analysis of the dynamic equilibrium states of the LotkaVolterra system suggests that the first step in the corresponding analysis for a more general model is to study the conditions for 
convergence of phase-plane trajectories. The general model of equation (4.1) can be rewritten in terms of new variables $z_{i}=\ln \mathrm{N}_{1}$ as

$$
\frac{d z_{i}}{d t}=F_{i}\left[\exp \left(z_{1}\right), \exp \left(z_{2}\right)\right]
$$

We consider two phase-plane trajectories of this general system which start from initial points $\vec{z}(0)$ and $\vec{z}(0)+\vec{x}(0)$ respectively, and which at any later time $t$ are at the points $\vec{z}(t)$ and $\vec{z}(t)+\vec{x}(t)$ respectively. From (4.9) we find equations of motion for the separation $\vec{x}(t)$ between trajectories as

$$
\frac{d \vec{x}}{d t}=\vec{F}[\exp (\vec{z}+\vec{x})]-\vec{F}[\exp (\vec{z})]
$$

Observe that $\vec{x}^{*}=(0,0)$ is an equilibrium point of $(4.10)$. Just as with the Lotka-Volterra system, we wish to examine the manner in which $|\vec{x}(t)|$ changes in time, and therefore need to determine whether or not $\vec{x}^{*}$ is a stable equilibrium point for the equations (4.10). In the Lotka-Volterra case we were able to establish global stabllity of the corresponding system (4.3) about $\vec{x}^{*}$. For the general system $(4.10)$ this cannot easily be done in a general way. We can however examine criteria for the local stability of (4.10) about the equilibrium point $\vec{x}^{*}$. If local stability about $\overrightarrow{\mathbf{x}}^{*}$ exists, we conclude that trajectories that start out with small initial separations will converge in time. This immediately raises the question of whether such a restricted convergence criterion will be sufficient to bring about dynamic equilibrium. We have tested the sufficiency of this criterion on various simple models and we find that it does work in many cases. We therefore proceed to a derivation of the criteria and devote the remainder of this section to examining their adequacy and usefulness. 
The neighborhood stability of $(4.10)$ about $\vec{x}^{*}$ can be examined in terms of the Routh-Hurwitz criteria (Hahn 1967). Essentially, we expand the functions on the right-hand side of $(4.10)$ about $\vec{x}^{*}$ assuming that $\left|\vec{x}(t)-\vec{x}^{*}\right|$ is small, and retain only first order terms. We get

$$
\frac{d x}{d t}=\left(\left.\frac{\partial \vec{F}}{\partial \vec{x}}\right|_{\vec{z}(t)} \mid \vec{x} .\right.
$$

The criterion for local stability is that the eigenvalues of the matrix $\left(\partial \vec{F} /\left.\partial \vec{x}\right|_{\vec{z}(t)}\right)$ have negative real parts. This gives us what we call the general convergence condi.tions

$$
\begin{gathered}
\mathrm{N}_{1}\left(\frac{\partial \mathrm{F}_{1}}{\partial \mathrm{N}_{1}}\right)+\mathrm{N}_{2}\left(\frac{\partial \mathrm{F}_{2}}{\partial \mathrm{N}_{2}}\right)<0, \\
\left(\frac{\partial \mathrm{F}_{1}}{\partial \mathrm{N}_{2}}\right)\left(\frac{\partial \mathrm{F}_{2}}{\partial \mathrm{N}_{1}}\right)<\left(\frac{\partial \mathrm{F}_{1}}{\partial \mathrm{N}_{1}}\right)\left(\frac{\partial \mathrm{F}_{2}}{\partial \mathrm{N}_{2}}\right),
\end{gathered}
$$

where the partial derivatives are evaluated along the trajectory $\vec{N}(t)$. The conditions (4.12) are intended to play the same role for general models that condition ( 4.7$)$ did for the Lotka-Volterra model.

Notice that the general conditions (4.12) when applied to the Lotka-Volterra model reduce to the condition for convergence (4.7). This is related to the fact that in the static equilibrium analysis on the Lotka-Volterra system (4.2), neighborhood stability about a static equilibrium point implies global stability about that point (Tuljapurkar \& Semura 1975). We will return briefly to the connection between the two types of analysis later in this section.

We now consider examples of models more general than LotkaVolterra and show that the general convergence conditions do guarantee the appearance of dynamical equilibrium states. Detalls of the 
numerical studies involved are not presented, as the methods used are identical to those discussed earlier in connection with the LotkaVolterra system. The various qualitative criteria discussed earlier concerning the magnitude of the parameters involved still hold good. With reference to the time scales discussed in setting up such criteria for $\mathrm{T}$ and $\mathrm{k}$, it is possible that a general model may not specify intrinsic growth rates $r_{i}$ explicitly. In such a case one takes the low biomass limits of the functions $F_{i}$ in the general model (4.1) to be equivalent to the $r_{i}$.

General Models

The first model we consider is one suggested by Maynard Smith (1974) for two-species competition. The model is intended to apply to situations where one or both of the competing species produces a substance toxic to the other, but only when the other is present. It is similar to the Lotka-Volterra model but has additional nonlinearities that are intended to mimic the behavior described. A static equilibrium analysis can be found in the discussion by Maynard Smith. The model can be written within the general form $(4.1)$ by spectfylng the functions $F$. We write these as

$$
F_{i}(N)=\frac{r_{i}}{K_{i}}\left(K_{i}-N_{1}-a_{i} N_{j}-b_{i} N_{1} N_{2}\right), i \neq j, i, j=1,2 .
$$

Applying the general convergence conditions (4.12), we obtain conditions for convergence of trajectories for the system (4.13) as

$$
a_{1} a_{2}<1, a_{1} b_{2} b_{1}, a_{2} b_{1}<b_{2} \text {. }
$$

These conditions as we pointed out in their derivation are local 
convergence conditions. In order to determine if they do in fact provide convergence that will drive the model into displaying a dynamic equilibriun state, we have performed numerical calculations for various choices of $T$ and $k$. In Figure 4 we show typical ratio convergence plots that indicate the approach to a dynamic equilibrium state. As in the Lotka-Volterra case the appearance of a dynamic equilibrium state is observed for wide ranges of $T$ and $k$, but is very sensitive to the limits In (4.14). Our conclusion is that in this model the conditions (4.14) are strong enough to guarantee the appearance of dynamic equilibrium states.

The second model we have studied has been used to describe predator-prey interactions. We use Leslie's (1948) form for the predator function, $F_{2}$, and the Lotka-Volterra form for the prey function, $F_{1}$. These are written as

$$
\begin{gathered}
\mathrm{F}_{1}=\frac{\mathrm{r}_{1}}{\mathrm{~K}_{1}}\left(\mathrm{~K}_{1}-\mathrm{N}_{1}-\mathrm{aN}_{2}\right), \\
\mathrm{F}_{2}=\mathrm{r}_{2}\left(1-\frac{\mathrm{N}_{2}}{\mathrm{bN_{1 }}}\right) .
\end{gathered}
$$

Here $\mathrm{N}_{1}$ and $\mathrm{N}_{2}$ are prey and predator biomasses respectively. Applying the general convergence conditions (4.12), we find that they reduce to the statement $\left(\mathrm{N}_{1}+\mathrm{aN}_{2}\right)>0$. This will hold in time-independent fashion for any $a>0$. We therefore expect that the model will display dynamic equilibrium states under periodic perturbations for all $a>0, b>0$. The condition on $b$ is built into the specification of the Leslie form $(4.15 b)$.

We have tested the above conclusion numerically over a wide range 
of the relevant parameters and find that it does hold. Figure 5 shows a typical ratio convergence plot for this system. The static equilibrium properties of this model are discussed by Maynard Smith (1974) and by Pielou (1969).

The examples we have discussed so far illustrate the appearance of dynamic equilibrium states in simple ecosystem models. The convergence conditions ( 4.12 ) seem to provide a satisfactory criterion. As the Leslie example illustrates, it is helpful if these criteria can be satisfied in some time-independent way. From the arguments in earlier sections, it is clear that the dynamic equilibrium state will be stable against a change in the parameters that define the perturbation. Thus if for any one period the kill factor $k$ is first changed in value and then is returned to its original value, the system will again tend towards the same dynamic equilibrium state which it would have tended toward originally. This stability essentially stems from the fact that the equilibrium state attained is independent of the initial biomasses of the two species.

Much of our analysis has been concerned with the determination of criteria that guarantee the convergence of phase-plane trajectories of simple models. We have not considered the question of where in the phase-plane these trajectories eventually converge. In general, they converge either toward a static equilibrium point or a limit cycle (if. one exists for the system). The connection between our analysis and the static equilibrium analysis follows easily if we think of the special case in which one of the two trajectories in our analysis is a static equilibrium point. Hence, as we pointed out earlier, the fact that local 


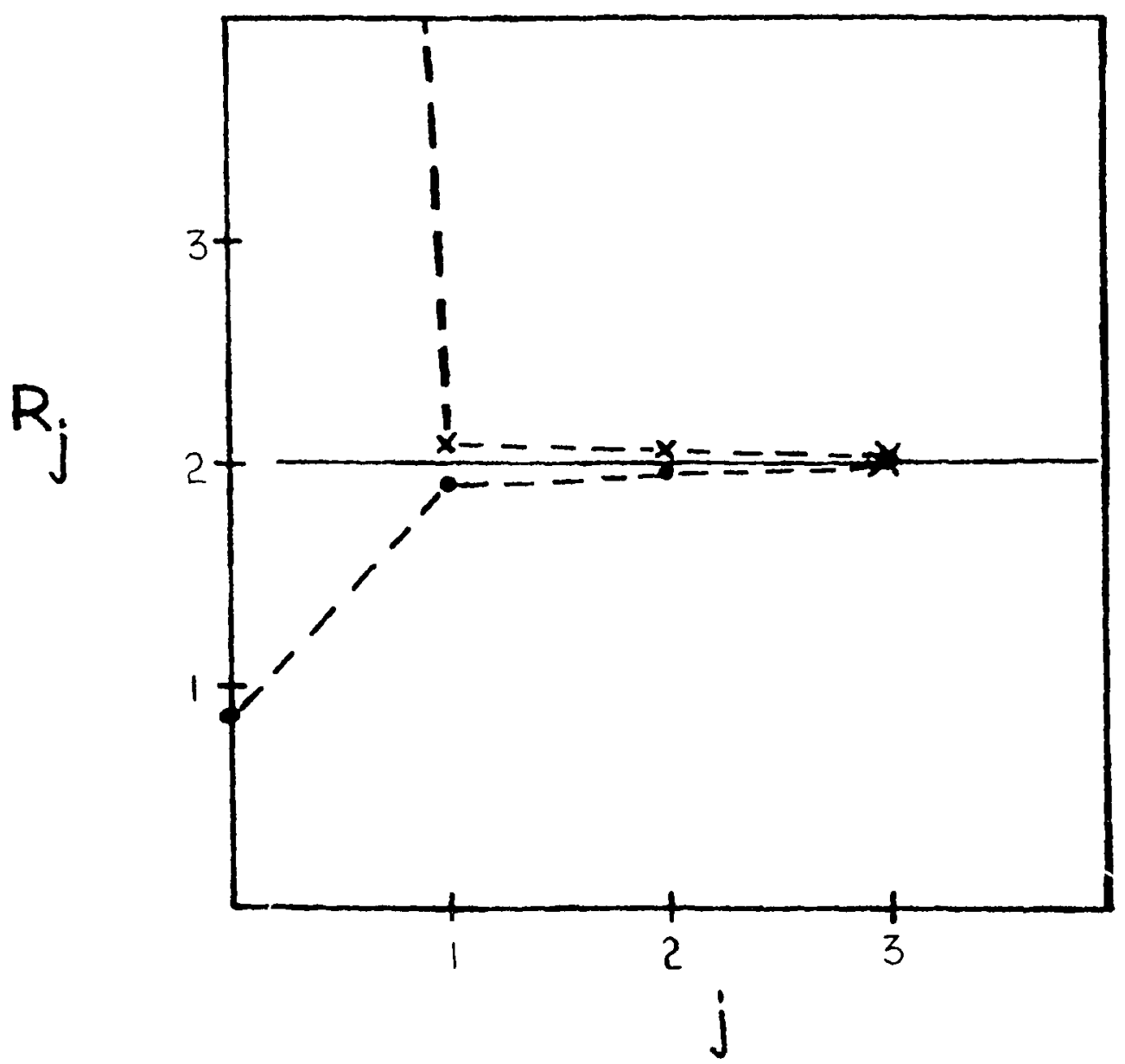

Figure 4. Biomass ratios for the competition model of Maynard Smith. Here $\mathrm{T}=20$ units; $\mathrm{r}_{2} / \mathrm{r}_{1}=2 ; \mathrm{a}_{1} \mathrm{a}_{2}=0.48 ; \mathrm{b}_{2} / \mathrm{b}_{1}=10$; $\mathrm{K}_{1} / \mathrm{K}_{2}=1$. Observe that the inltial condrtions (equal biomass) lead to widely different initial biomass ratios. In fact species 2 (dots) shows a decline in biomass initially. 


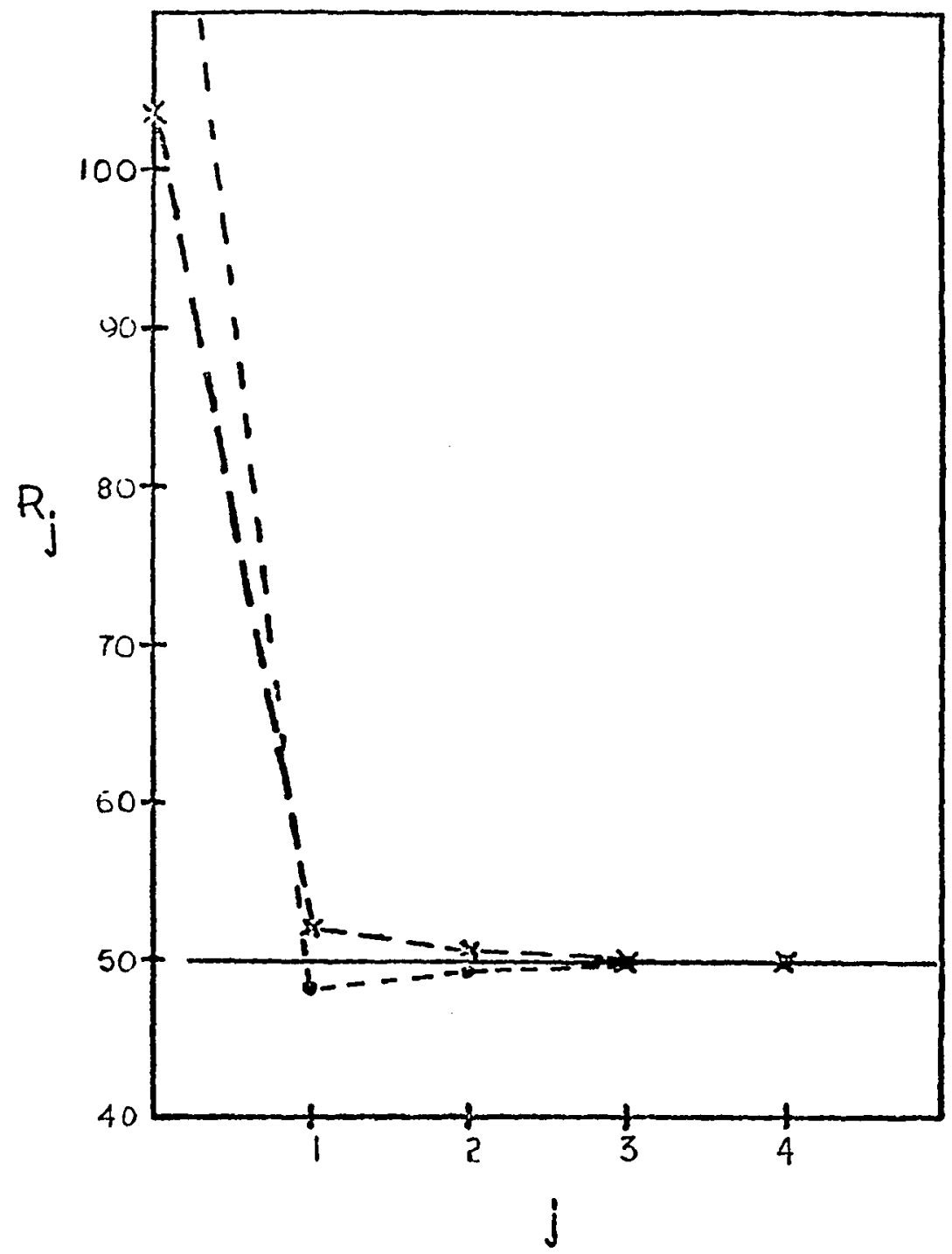

Figure 5. Biomass ratios for Leslie's predator-prey model. Here $T=10$ units; $a b=0.48 ; r_{1} / r_{2}=3$. As in Figure 4 the ratios converge to $k$ in a small number of iteration steps. 
stability about a static equilibrium implies global stability for the Lotka-Volterra system (4.2) leads directly to the fact that $10 \mathrm{cal}$ and global convergence results are also equivalent.

The general convergence criteria (4.12) show that self-regulation is an important factor in making dynamic equilibrium states possible. This echoes the theme that self-regulation tends to stabilize simple models about static equilibrium points. The criterion ( $4.12 b$ ) that we have obtained follows, incidentally, from two of the criteria that guarantee the existence of a stable static equilibrium point or a limit cycle in simple models. The latter criteria are those of the Kolmogoroff theorem, and are discussed by May (1974).

\section{DISCUSSION}

We are aware of the ecological deficiencies of the various models we have considered. However, the point being made here is that the general convergence conditions seem to provide rather robust criteria for the convergence of trajectories in simple models. Given these conditions, the appearance of dynamical equilibrium states appears to be a general feature of simple models. The ecological implication is that simple ecosystems may persist or be maintained in an equilibrium state by strong periodic perturbations, and that this state is not a static equilibrium state.

The dynamical equilibrium states we describe are simflar in spirit to Hutchinson's (1953) concept of nonequilibrium communfties. Hutchinson's example of the coexistence of similar species of birds in the Arctic, where local populations can easily be exterminated by 
adverse climatic conditions (Wynne-Edwards 1952), favors an identification of the two concepts.

The perturbations we consider may also, as suggested by Koch (1974), be viewed as representing some form of seasonal mortality effect. The periods involved can then be identified with season lengths. In such a case, one can think of dynamic equilibrium as a simple paradigm for a 'seasonal mode' of existence as opposed to an 'equable mode' of existence (Stewart \& Levin 1973).

As an example of the ecological relevance of the dynamic equilibrium analysis, we examine the effect of periodic grazing or defoliation on vegetational diversity. Consider a group of species growing in the same field, and assume that these species may be ranked by competitive ability into a mix of dominant and weaker species. In the absence of any perturbations, the mix of species would tend towards a static equilibrium state, where it is 11kely that only dominant species would survive. Now suppose that the original mix of species is subjected to periodic grazing or defoliation, and also that the impact of grazing or defoliation is proportionally the same on all species. According to our analysis, such a periodic perturbation ought to produce a dynamic equilibrium state, where the chances of weaker species coexisting with dominant ones ought to be greater than the corresponding chances of coexistence in a static equilibrium state. Therefore periodic grazing or defoliation which has proportionally equal impact on the species growing in a field ought to lead to greater vegetational diversity. This conclusion is consistent with observations on vegetational diversity as reviewed by Harper $(1967,1969)$. The effect of grazing or 
defoliation which affects different species to different degrees would require an analysis using different kill factors for the different species. However, the central concept of dynamical equilibrium states appears to provide an essentially correct picture of the impact of periodic grazing or defoliation. In this context, harvesting is a relevant type of perturbation. A recent analysis by Brauer \& Sanchez (1975) examines harvesting in terms of a constant reduction in species biomass per unit time.

One of the useful features of 'strategic' concepts in ecology is that they provide a well-defined language that is applicable to examples other than simple ecosystems. The perturbations that we have considered here are strong exogenous perturbations that have a time scale long compared with the intrinsic growth times scales of the interacting species. Analogous perturbations can be found in complex ecosystems. For example, in large ecosystem models there may be environmental variables that enter exogenously into the equations of motion of the subsystems, and also have periodicities that are long compared to the time scales that characterize the subsystems. These may be thought of as imposing a deterministic periodic perturbation on the variables that describe the subsystems. An example of this situation is discussed by Parker (1975) in the context of modeling studies of lakes. The equilibrium states of the model he discusses are analogous to the dynamic equilibrium states that we have described.

The discrete periodic perturbations we have considered can only be applied exogenously to ecological communities. Our analysis para1lels a control problem, as in the cases of harvesting or exogenous 
environmental variation we have discussed. These examples and analogies demonstrate that there may be a case for dynamic equilibrium to play a 'strategic' role in ecological theory. This role might complement the central role that static equilibrium analysis has thus far played in the theory. Concepts such as dynamic equilibrium would be a step towards the study of the nonequilibrium dynamics of ecological communities. 
CHAPTER V

STOCHASTIC STABILITY AND LIAPUNOV STABILITY

INTRODUCTION

Fluctuations in numbers are very often characteristic of the dynamics of natural populations. Theoretical descriptions of population dynamics have therefore attempted to incorporate the essential aspects of temporal variability in population numbers. The central concern of this chapter is the analysis of temporal fluctuations in continuous nonlinear population models which are made stochastic by the 'addition of white noise'. We show that the qualitative behavior of nonlinear inodels with white noise is determined by a Liapunov function for the initial nonlinear deterministic model. Our analysis, which starts from the work of Ludwig (1975), shows that this result is valid for small white noise perturbations of general nonlinear models and is independent of the choice of stochastic calculus. Liapunov stability of a deterministic model therefore provides sensible qualitative information on the effects of including white noise, in spite of the technical difficulties recently stressed (May 1974, Feldman and Roughgarden 1975)

The discussion in this chapter has three main thrusts. We begin by considering the important matter of the time-scales involved in setting up a dynamical theory of interacting populations. This provides useful criteria concerning the nature of noise and enables us to relate 
models with white noise to alternative stochastic formulations of population dynamics.

Next we turn specifically to nonlinear continuous models which possess a stable equilibrium and examine the effects of adding white noise. Following Ludwig (1975; henceforward referred to as DL 75) the question of boundary conditions on the stochastic problem is analyzed. We show that Ludwig's choice of boundary conditions is in many ways a 'natural one' and is consistent with the boundary conditions used in alternative mathematical formulations of stochastic population models. With these boundary conditions, a solution procedure is formalized and a measure of stability for the stochastic model is defined. The solution procedure is now applied to the problem of small stochastic perturbations and the result relating stochastic stability with deterministic Liapunov stability is established.

Following this proof we use our result to examine current ideas concerning the relationship between the geometry of Liapunov functions and stability in the presence of noise (Holling 1973, Gilpin 1974). Liapunov functions have implicitly and explicitly been thought of as providing a 'dynamical landscape' over which a nonlinear system moves. For models with a stable equilibrium the Liapunov function is a sort of 'bowl' centered at equilibrium. The geometry of this bowl is assumed to provide qualitative information about the motion of the system in the presence of noise. We consider the simple case of symmetric twospecies Lotka-Vo1terra competition and display no less than four distinct Liapunov functions for the system. The geometry of these four functions is qualitatively different, and only one function is known 
to provide information on the stochastic problem. We conclude that considerable caution is advisable in using the geometry of an arbitrary Liapunov function as a guide to stochastic stability.

The concluding discussion considers first the limitations and the role of models incorporating white noise, as well as other related stochastic models, in light of the 'extinction nightmare' stressed by Korostyshevsky et. al. (1974). We also briefly discuss the role of models with a single equilibrium vis-a-vis models with many equilibria, models where equilibrium points are replaced by regions or domains of attraction (Holling 1973), and models which stress the nature of historical sequence rather than equilibrium (Botkin and Sobel 1974).

The mathematical detalls of several points made in the chapter are presented in Appendices. This allows us to concentrate on the main thread of our arguments without tedious mathematical detours and to state concisely purely mathematical arguments.

THE NATURE OF NOISE

\section{Time Scales and Models}

The creation of a model of interacting populations requires the corsideration of two sets of time scales. The first set of time scales broadly separates the types of change which occur in natural populations in terms of typical rates at which these changes take place. A possible set of time scales of this first type for populations is, in increasing order: physiological (times over which changes in an organism's physical state occur), generation times, genetic, geological, astronomical. The ecological models of populations studied in this 
chapter are taken to be relevant over times significantly longer than physiological time scales, but definitely shorter than genetic time scales; this range contains ecological time scales.

This restriction of ecological time scales strongly constrains the details of any models we may construct and is particularly important when we attempt to relate the behavior of models to phenomena in the real world. It should be clear that our specification of time scales is a theoretical construct. Real phenomena may not respect such a separation of time scales. For instance genetic time scales may turn out to be short (only a few generation times), as with some examples of competing Drosophila (Ayala 1969) or of predator-prey interaction (Pimentel 1968). But situations of the latter sort require that we either seek new models or reconsider old ones (Roughgarden 1976).

A second set of time scales becomes relevant once it is decided to build an ecological model of an ecosystem or a set of populations. This second set is the collection of characteristic time scales of all the dynamical variables in the ecosystem. The formulation of a mathematical model requires the selection of dynamically and empirically sufficient variables from those available (Lewontin 1972). For example, the models we consider in this paper primarily use population numbers as dynamical variables. Such a selection usually excludes from the model the dynamical effects of other variables whose characteristic time scales differ from the time scales of the model variables. The principal basis for working with a small selected set of variables in a model is the assumption that variables possessing longer time scales change slowly enough to be regarded as effectively constant, while 
variations on much shorter times scales are small enough to be neglected as a first approximation (Simon 1973). The perturbations to the basic model caused by variations on time scales shorter than the model variables are collectively called 'noise'. The separation of time scales and the small amplitude of noise deserve to be strongly stressed (See Papanicolau 1972 for an exposition of the mathematical significance of time scales).

We now apply the preceding discussion to continuous stochastic models and show that biologically and mathematically significant conditions on the models emerge from a consideration of time scales.

Stochastic Population Models Involving Noise

The effects of noise are usually included in population models by defining the model in terms of some type of stochastic process. For a single species whose population size is $\mathrm{N}$, a general continuous stochastic model is

$$
\frac{d N}{d t}=G[N(t)]+F[N(t), Z(t)] \text {, }
$$

where $Z(t)$ is a stochastic process of known properties describing noise, and the function $F$ accounts for the way the $N(t)$ and $Z(t)$ processes interact. It is mathematically convenient to assume that the noise $\mathrm{Z}(\mathrm{t})$ is a stationary Markov process with zero mean value, a finfte variance $\sigma^{2}=E[Z(t) Z(t)]$, and a finfte correlation time

$$
\tau=\int_{0}^{\infty} \Gamma(s) \mathrm{ds}, \Gamma(s)=\frac{1}{\sigma^{2}} E[Z(t+s) Z(s)] \text {. }
$$

The quantity $\tau$ is the characteristic time scale for the noise, while $\sigma^{2}$ indicates the 'strength' of the noise. The function $F$ is taken to have 
zero mean value for fixed $N$. The deterministic analog of (5.1) is the model

$$
\frac{\mathrm{d} \mathfrak{n}}{\mathrm{dt}}=G[\mathrm{n}(\mathrm{t})],
$$

where the lower case letter indicates a deterministic variable. The starting point of our study is actually the deterministic model (5.3) to which noise is added to produce (5.1). From (5.3) it is possible to define an appropriate characteristic time scale for the variation of population size in the absence of noise; call this time scale $T$.

The usual strategy for the analysis of temporal variations assumes that the deterministic model (5.3) provides at least a reasonable description of population change. The noise is assumed to satisfy the two conditions of the previous subsection, namely (a) $\tau<<T$ (separation of time scales), (b) $\sigma^{2}$ is in some sense small (the noise has small amplitude). (It is worth pointing out that if the stochastic model (5.1) is taken as the starting point, then for a nonlinear function $G$ the deterministic mode1 (5.3) does not describe the evolution of mean values $n=E[N]$. This is why (5.3) is only an analog of (5.1).)

We now consider briefly the physical and biologlcal origins of the noise $Z(t)$. One source of noise is environmental fluctuations (May 1974, Roughgarden 1975). Other sources are suggested by G11pin (1974). An important point to bear in mind is condition (a) above. The quantity $T$ definitely lies in the range of ecological time scales discussed previously. Therefore the only sources of noise which the present formalism can accommodate should have time scales $\tau$ much shorter than ecological time scales. For many species, seasonal changes in the environment will not satisfy this constraint. Another example is 
that of periodically recurrent epidemics which often have time scales longer than $T$. Temporal variations due to disturbances such as the last two are treated most effectively by modifying the deterministic system, not by adding noise (Papanicolau 1972).

In the next two sections we will adopt the rather drastic simplification of letting $\mathrm{Z}(\mathrm{t})$ be 'white' noise, or in other words, taking the limit $\tau \rightarrow 0$. This limit makes the stochastic model (5.1) amenable to treatment by diffusion theory (Feller 1952). In an important sense the reason for working with white noise and diffusion Markov processes stems from the fact that the deterministic models studied here are assumed to possess a single equilibrium point. Thus it is hoped that diffusion theory will yield a meaningful equilibrium probability distribution which can be compared with the equilibrium-centered deterministic behavior. As we will see this hope dors not always seem to be justified. If the deterministic models merely predicted specific time paths for given initial conditions (instead of an equilibrium which attracts all time paths), we could keep $\tau$ finite and study changes caused by noise in these time paths. Powerful perturbation methods to do this are now available (Papanicolau and Kohler 1974, Lax 1966).

We conclude this section with a comment on the mathematical approaches to stochastic models. Our limitation to Markov processes is traditional: besides analytical tractability and wide applicability, Markov processes have a common mathematical thread tying together different formulations (such as discrete and continuous models; Bart-lett 1973). It is worth remembering that a model specified by, say, a set of transition probabilities is equivalent to a differential 
(Langevin) equation model incorporating white noise (Lax 1966). Such relationships allow a reasonable comparison between stochastic models formulated in very different ways.

In the next section we consider directly general models incorporating white noise, and establish a relationship between stochastic stability in such models and deterministic Liapunov stability.

\section{STABILITY IN MODELS WITH WHITE NOISE}

\section{Adding White Noise to Models}

We set out here the general form of the stochastic models to be studied and outline the sequence of steps in the subsequent analysis of this section. The starting point is a deterministic model for m species, written in terms of the m-vector $\vec{n}(t)$ of population numbers $n_{i}(t)$,

$$
\frac{\mathrm{d} \overrightarrow{\mathrm{n}}}{\mathrm{dt}}=\overrightarrow{\mathrm{G}}[\overrightarrow{\mathrm{n}}(\mathrm{t})]
$$

It is assumed that model (5.4) possesses a stable equilibrium at $\overrightarrow{\mathrm{n}}=$ $\vec{n} *$, with all $\mathrm{n}_{\dot{\mathrm{i}}}^{*}, i=1, \ldots, \mathrm{m}$, positive. From this point on it will be convenient to work with population sizes measured relative to equilibrium, i.e., with the vector $\vec{x}(t)=\vec{n}(t)-\vec{n}^{*}$. We rewrite $(5.4)$ as

$$
\frac{d \vec{x}}{d t}=\vec{G}[\vec{x}(t)]
$$

We describe the white noise heuristically as a k-component 'white noise process' $\vec{Z}(t)=\left[Z_{1}(t), \ldots, Z_{k}(t)\right]$, with the properties

$$
\begin{aligned}
E\left[z_{i}(t)\right] & =0, \\
E\left[z_{i}(t) z_{j}(t)\right] & =d_{i j} \delta\left(t-t^{\prime}\right), \\
1, j & =1, \ldots, k .
\end{aligned}
$$


If the $k$ processes $z_{1}(t)$ are uncorrelated, we assume $d_{1 j}=\delta_{1 j}$; if they are correlated, we assume $d_{1 i}=1,\left|d_{1 j}\right|(1 \neq j) \leq 1, i, j=1, \ldots, k$. (It is not really proper mathematically to define a process with properties (5.6). However, there are two rigorous ways of representing white nolse, so we here use $(5.6)$ as containing the essence of both rigorous formulations and present details of both in Appendix B).

In general white noise affects the dynamics of (5.5) in some density-d $=$ iendent way, so we introduce functions $F_{1 j}(\bar{x}), 1=1, \ldots, m, j=$ $1, \ldots, k$, which incorporate the density-dependence. A general stochastic model for the population process $\vec{X}(t)$ may now be written as

$$
\begin{aligned}
& \frac{d \vec{X}}{d t}=\vec{G}[\vec{X}(t)]+\sqrt{\varepsilon} F[\vec{X}(t)] \vec{Z}(t), \\
& \frac{d X}{d t}=G_{1}(\vec{X})+\sqrt{\varepsilon} \sum_{j=1}^{k} F_{1 j}(\vec{X}) Z_{j}(t) .
\end{aligned}
$$

In (5.7a) $F$ is the (m $x k$ ) matrix of functions $F_{1 j}$. The quantity $\varepsilon$ is a parameter introduced in DL 75 for the following purpose. In view of the properties of the white noise process, the magnitude of the variance-covarlance matrix of the nolse terms in (5.7) is proportional to $\varepsilon$. Therefore $\varepsilon$ governs the strength of the noise. If $\varepsilon=0$ in (5.7) we regain the deterministic model (5.5).

The behavior of the stochastic process $\vec{X}(t)$ is conveniently described in terms of the probability density function $f(\vec{x}, t)=f\left(x_{1}\right.$, $\left.\ldots, x_{m}, t\right)$ which gives the probability for $\vec{x}(t)$ to lie between $\vec{x}$ and $\vec{x}+d \vec{x}$ to be $f(\vec{x}, t) d \vec{x}$. The density $f(\vec{x}, t)$ for (5.7) obeys a partial differential equation called the diffusion equation. Both the rigorous forms of (5.7) yleld diffusion equations, which are set out in Appendix B. In efther case, the general form of the equation is as follows: 
$\frac{\partial f(\vec{x}, t)}{\partial t}=L_{*} f(\vec{x}, t)=\frac{\varepsilon}{2} \sum_{i, j=1}^{m} \frac{\partial^{2}}{\partial x_{i} \partial x_{j}}\left(a_{i j}(\vec{x}) f(\vec{x}, t)\right)-\sum_{i=1}^{m} \frac{\partial}{\partial x_{1}}\left(b_{i}(\vec{x}) f(\vec{x}, t)\right)$

Here $L_{*}$ is the partial differential operator written out explicitly on the right. The asterisk takes the place of a subscript, I or S, according as the Ito calculus (Doob 1953) or the Stratonovitch-Papanicolau-Kohler formulation (Stratonovitch 1966, Papanicolau and Kohler 1974) is used to arrive at (5.8). The quantities $a_{1 j}(\vec{x}), b_{1}(\vec{x})$ are the diffusion and drift coefficients respectively, and are written out in Appendix A. For the present we note that the $a_{i j}(\vec{x})$ are the same for both the $L_{I}$ and the $L_{S}$ operators. Further, the $a_{i j}(\vec{x})$ are defined as the elements of variance-covariance matrix, and so the matrix $A=\left(a_{i j}\right)$ has a nonnegative symetric part.

The rest of this section constitutes an Investigation of solutions of the diffuston equation (5.8). The analysis draws extensively on DL 75 and we refer to that paper for details of some proofs. First, however, we set forth one important assumption and outline the plan of attack:

1. The 'small $\varepsilon$ ' 1 imit. In accordance with our earlier discussions we emphasize the small relative strength of noise in the model (5.7). Additionally we are interested mainly in characterizing the qualitative effects of small nolse. Therefore we will work in the limit where $\varepsilon$ is small.

2. Boundary conditions. A unlque solution to (5.8) can only be found if appropriate boundary conditions are prescribed. This question is considered in the next section, along with the question of the 
existence of an equilibrium density $f^{*}(\vec{x})=\lim _{t \rightarrow \infty} f(\vec{x}, t)$.

3. Solution procedure. Following a selection of boundary conditions, we present a solution procedure for (5.8) and define a measure of stability for the stochastic model (5.7).

This sequence of arguments enables us to establish our final result towards the end of this section.

\section{Boundary Conditions}

The objective here is to define the range of population values over which the stochastic process $(5.7)$ describes the dynamics of the populations, and the behavior of the process at the boundarles of this range.

First it is assumed that there 1s some upper limit to the values which the population numbers can take. Thus the process $\vec{X}(t)$ has some arbitrary finite upper bound. The precise population numbers which define the upper bound are not important. Blologically the upper bound follows quite reasonably from the Idea that all populations are ultimately Iimited by finite resources. Mathematically the upper bound is specifled as a boundary $R$, a surface in the m-dimenstonal space of population numbers $\vec{n}$. The boundary $R$ is clearly impenetrable for the process $\overrightarrow{\mathrm{X}}(t)$.

The second and more important question is of a lower boundary for $\vec{X}(t)$. It is generally recognized that the treatment of population numbers $\vec{n}$ as continuous variables is an approximation valid only for large populations. When populations are small it is necessary to recognize that $\vec{n}$ is a discrete variable representing counts of individuals. We therefore assume that the continuous model (5.1) and the associated 
stochastic model (5.7) are valid only when population sizes are large. To make this precise, a lower bound 8 is chosen such that (5.1) and (5.7) are valld only when individual population sizes $n_{1}, 1=1, \ldots, m$, exceed $s$. For $n_{i}<s$, it is necessary to use a discrete- $\vec{n}$ model. The hyperplanes $n_{1}=s, i=1, \ldots, m$, define a lower boundary for the stochastic process $\vec{X}(t)$. We call this lower boundary $\Sigma$.

In order to determine the nature of the boundary $\Sigma$, consider discrete population models which replace (5.7) inside $\Sigma$. The usual discrete stochastic model takes the form of a Markov chain with a denumberable set of accessible states. Typlcal examples are models analogous to continuous models such as the Lotka-Volterra model (Botkin and Sobe1 1974, Reddy 1974). The feature of such discrete models of primary Importance here is that the state $\vec{n}=\overrightarrow{0}$ (or the states $n_{1}=0$ for one or more $1, i=1, \ldots, m)$ are always absorbing states. In a discrete Markov model this feature Implies that one or more populations w111 with high probability go extinct over long times. (Reddy 1974 has a simple proof; or see Karlin 1974, Korosyteshevsky et. al. 1974.)

We conclude that $\Sigma$ is an absorbing boundary for $\overrightarrow{\mathrm{X}}(t)$ in the sense that extinction is certain if the process reaches $\Sigma$. Therefore the process $\vec{X}(t)$ is to be studied In the region of population values lying between the absorbing extinction boundary $\Sigma$ and the impenetrable upper boundary $R$.

At this point we direct attention to a related but different feature of the stochastic model (5.7). The density-dependence of the noise in this model is described by the functions $F_{1 j}(\vec{x})$, which are also, of course, functions of $\vec{N}$. In view of previous work (Leving 1969, 
Kiester and Barakat 1972, G1lpin 1974, May 1974, Feldman and Roughgarden 1975, Abrams 1975) the functions $F_{1 j}$ are typically assumed to be of the form $\mathrm{N}_{1}^{\mathrm{b}}$, where $\mathrm{b}$ is some number. Therefore it seems generally true that the $\mathrm{F}_{i j}$ are taken to be nonzero at equilibrium, and hence the equilibrium $\vec{n}^{*}$ is not an absorbing state for the process $\vec{x}(t)$. In consequence the boundary $\Sigma$ contains all the absorbing states for $\vec{X}(t)$.

In view of the foregoing arguments we find (as in DL 75) that the process $\vec{x}(t)$ cannot have a nontrivial equilibrium distribution. If we wait long enough $\vec{x}(t)$ will, with probability near one, reach $\Sigma$ and one or more populations will go extinct. To put the matter mathematically, $\lim f(\vec{x}, t)=0$. Given this behavior it is no longer relevant to worry $t \rightarrow \infty$ about the equilibrium distribution. Rather, we must shift attention to the question of how long the process $\vec{X}(t)$ takes to reach $\Sigma$ (with probability near one), starting from some initial state.

The recognition of these features of the $\vec{X}(t)$ process is the critical step here and in the arguments of Ludwig (1975). In the subsequent analysis the results we draw from $D L 75$ constitute a powerful application and extension of the results of M1ller (1962) and Vent'sel and Friediln (1970).

Before going any further we stress that the notion of ultimate extinction is mathematically rather than biologically significant. Several population models display the feature of extinction: models of type (5.7) are found in May (1974), Maynard Smith (1974); the stochastic version of the Leslie matrix model (Namkoong 1974); general discrete Markov chain models (Korosyteshevsky et. al. 1974); continuous population discrete time models (Poole 1974); models of gene fixation (DL 75, 
Kimura 1955). However, it is easy enough to build models which behave differently, and we discuss such examples together with the biological relevance of extinction in a later section. A last point 1s that the use of an absorbing barrier $\Sigma$ for small finite $\vec{n}$ has been suggested independently of our discussion of discrete models (Botkin and Sobel 1974).

\section{A Solution Procedure}

The question of how long the process $\overrightarrow{\mathrm{X}}(t)$ takes to reach the $a b-$ sorbing boundary $\Sigma$ may be restated as: how does the probability density $f(\vec{x}, t)$ decay in time? The time development of $f(\vec{x}, t)$ is studied by using an eigenfunction expansion (Kimura 1955, Bailey 1962). A set of eigenfunctions $f_{k}(\vec{x})$ and corresponding elgenfunctions $\lambda_{k}$ are found for the diffusion equation (5.8) such that $L_{*} f_{k}(\vec{x})=-\lambda_{k} f_{k}(\vec{x})$. The density $f(\vec{x}, t)$ is then given by a combination of the form $f(\vec{x}, t)=\sum_{k} a_{k} f(\vec{x}) e^{-\lambda_{k} t}$ where the $a_{k}$ are numbers. Let the smallest eigenvalue of the diffusion operator be $\lambda_{0}=\left\{\right.$ MIn. $\left.\lambda_{k}\right\}$. Then as $t \rightarrow \infty$ the behavior of $f(\vec{x}, t)$ is dominated by the exponential $e^{-\lambda_{0} t}$. Asymptotically the decay rate of $f(\vec{x}, t)$ is governed by $\lambda_{0}$, and the decay times are roughly exponentially distributed. The expected time for the density to decay is therefore $T_{0}=\frac{1}{\lambda_{0}}$. If the process $\vec{X}(t)$ starts from some Initial point $\vec{x}_{0}$, then $\mathrm{T}_{0}=\frac{1}{\lambda_{0}}$ gives the expected time after which $\overrightarrow{\mathrm{X}}(\mathrm{t})$ w111 have reached $\Sigma$ with probability near one (Vent'sel and Friedlin 1970).

Our objective now becomes the calculation of the minimum eigenvalue $\lambda_{0}$ from the diffusion equation $(5.8)$. The quantity $T_{0}=\frac{1}{\lambda_{0}}$ provides a measure of stability for the stochastic model (5.7). Ludwig 
(DL 75) calls $T_{0}$ the 'persistence' of the process $\vec{x}(t)$. The biological meaning of $T_{0}$ will be considered in more detail later, but for the present its significance to the white noise problem is clear. The method to be presented for obtaining $T_{0}$ was developed by Miller (1962), applied to population models in $\mathrm{DL} 75$, and has applications in physical problems (Weiss \& Dishon 1975).

As previously remarked, we will concentrate on the case where the noise is small, $1 . e .$, the small- $\varepsilon$ 1Imit. The process $\vec{X}(t)$ is assumed to start at equilibrium, $\vec{x}=0$. When $\varepsilon$ is small it is expected that for long times the sample paths of $\vec{x}(t)$ will be found mostly near the stable equilibrium. The boundary $\sum$ is reached when large devlations from equilibrium occur. For long times, the density $f(\vec{x}, t)$ decays at a rate given by $\lambda_{0}$, and $\lambda_{0}$ will be small when $\varepsilon$ is small. Thus $f(\vec{x}, t)$ is visuallzed as being concentrated around $\vec{x}=\overrightarrow{0}$ and varying slowly in time. Hence we adopt the following procedure to determine $\lambda_{0}$ : first we solve the tIme-independent problem $L_{*} g(\vec{x})=0$, and seek a solution $g(\vec{x})$ which is concentrated at the equilibrium $\vec{x}=0$; next we use $g(\vec{x})$ to estimate $\lambda_{0}$ in the eigenvalue problem $L_{*} f_{0}(\vec{x})=-\lambda_{0} f_{0}(\vec{x})$. The power of the procedure rests on the introduction of an expansion in the small parameter $\varepsilon$, which makes the estimation of $\lambda_{0}$ accurate to order $\varepsilon$.

Following DL 75, the time-independent problem is recast around Ludwig's ansatz:

$$
g(\vec{x})=\exp \left[-\frac{v(\vec{x})}{\varepsilon}\right] h(\vec{x})
$$

so that for small $\varepsilon$ the function $v(\vec{x})$ dominates $g(\vec{x})$, and $h(\vec{x})$ is essentially a correction term. It is required that $v(\vec{x})$ have a minimum at the equilibrium $\vec{x}=\overrightarrow{0}$ and increase away from it, so that $g(\vec{x})$ is 
concentrated at equilibrium. The ansatz (5.9) is now inserted into the time-independent problem $L_{*} g(\vec{x})=0$, and the resulting equation is grouped by terms proportional to different powers of $\varepsilon$. For small $\varepsilon$, the most significant term is proportional to $\frac{1}{\varepsilon}$, the next to $\varepsilon^{0}$, and so on. To obtain results of accuracy $\varepsilon$ only the first two terms are retained, and are separately set equal to zero. Using the general form $(5.8)$, and (5.9), the most important equation determines $v(\vec{x})$ as the solution of

$$
\frac{1}{2} \sum_{i, j} a_{i j} \frac{\partial v}{\partial x_{i}} \frac{\partial v}{\partial x_{j}}+\sum_{i} G_{1}(\vec{x}) \frac{\partial v}{\partial x_{1}}=0, \quad 1, j=1, \ldots, m .
$$

Here we have used the explicit forms of $b_{1}(\vec{x})$, the drift coefficients, from Appendix B. Note that equation (5.10) for $v(\vec{x})$ is independent of the cholce of stochastic calculus.

The solution $v(\vec{x})$ of $(5.10)$ is used to find $h(\vec{x})$ (the equation for $h(\vec{x})$ is written out in Appendix $C$. The resulting functions form $g(\vec{x})$, which is used to estimate $\lambda_{0}$ (following Miller 1962) to order $\varepsilon$. It 1s found (DL 75) that

$$
\lambda_{0}=\mathrm{K}\left(\Sigma^{*}\right) \exp \left[-\frac{\mathrm{v}\left(\Sigma^{*}\right)}{\varepsilon}\right]
$$

where $\Sigma^{*}$ stands for coordinates on $\Sigma$ where $v(\vec{x})$ hes its minimum value, and $K\left(\Sigma^{*}\right)$ consists of boundary contributions and a normalization term which are detalled in Appendix C.

Vent'sel and Friedlin (1970) and DL 75 present elegant discussions of this entire procedure in terms of an action functional for the stochastic process $\overrightarrow{\mathrm{x}}(t)$. Here we emphasize the following points:

1. The equation (5.10) is independent of the stochastic calculus used, 
2. for small $\varepsilon, \lambda_{0}$ ls mainly determined by the value of $v(x)$ on $\Sigma$,

3. the entire procedure leading to (5.11) rests on the assumption of $\operatorname{small} \varepsilon$.

Further mathematical detalls of the solution of (5.10) and the result (5.11) are given in Appendix C. Certain aspects of the analysis there w111 be needed in the next subsection, but the presentation in this section covers the essential points of the solution procedure. We next consider the nature of the function $v(\vec{x})$ and show that $(5.11)$ expresses a relationship between a deterministic Liapunov function and stochastic stability as expressed by $\lambda_{0}$.

\section{A Liapunov Function}

The discussion up to this point shows that the temporal behavior of the general model (5.7) incorporating white noise is characterized by the minimum elgenvalue $\lambda_{0}$, and that $\lambda_{0}$ is largely determined by the solution $v(\vec{x})$ of $(5.10)$. We now show that $v(\vec{x})$ is in fact a Liapunov function for the full nonlinear deterministic model (5.4) about the equilibrium $\vec{x}=\overrightarrow{0}$.

The proof of this point follows from the properties of $v(\vec{x})$ and its defining equation (5.10), as set out below:

1. As pointed out following equation (5.9), it is required that $v(\vec{x})$ have a minimum at $\vec{x}=\overrightarrow{0}$ and be increasing in the nelghborhood of equilibrlum. In fact we can quite generally demand that $\underline{v(\vec{x})}=0$ at $\vec{x}=\overrightarrow{0}$, and is strictly increasing around equilibrium.

2. Subject to the conditions Iisted in 1 above, the equation (5.10) is solved to find $v(\vec{x})$. The solution is obtained by replacing 
(5.10) with an equivalent system of ordinary differential equations, which yield solution curves $x_{1}(\sigma), v(\sigma), 1=1, \ldots, m$, where $\sigma$ is a parameter along the curves (see Append1x C). The solution curves start near $\vec{x}=0$ with $\sigma=0$ and move away from equilibrium to cover $\vec{x}$-space as $\sigma$ increases. Along each solution curve for every $\sigma>0$ we know $\vec{x}(\sigma)$, $v(\sigma)$, and so we get $v(\vec{x})$. As shown in DL 75 (also Appendix C), as long as the solution procedure works, $v(\vec{x})$ is nondecreasing or increasing on every solution curve. Therefore $\mathrm{v}(\overrightarrow{\mathrm{x}})$ is a positive-definite function which increases (or at least is nondecreasing) away from $\vec{x}=\overrightarrow{0}$.

3. Finally consider the time derivative o. $(\dot{\bar{x}})$ along the trajectories of the deterministic system (5.4),

$$
\frac{d v(\vec{x})}{d t}=\sum_{1=1}^{m} G_{1}(\vec{x}) \frac{\partial v}{\partial x_{1}}
$$

Using (5.10) we can write this as

$$
\frac{d v}{d t}=\sum_{i=1}^{m} G_{1}(\vec{x}) \frac{\partial v}{\partial x_{i}}=-\sum_{1, j=1}^{m} a_{i j} \frac{\partial v}{\partial x_{i}} \frac{\partial v}{\partial x_{j}}
$$

Recall from the discussion following the diffusion equation (5.8) that $a_{i j}$ has a nonnegative symmetric part. Then we see that $\frac{d v}{d t} \leq 0$ along the trajectories of $(5.4)$.

The properties $1,2,3$ above ensure that $v(\vec{x})$ plays the role of a Liapunov function for the deterministic model (5.4). There are two questions that arise immediately concerning the nature of $v(\vec{x})$ viewed as a Liapunov function, and we address these in turn.

First, does $v(\vec{x})$ describe the global stability behavior of the deterministic system (5.4)? It does not seem possible to answer this question without examining speciflc cases. The answer really depends 
on the existence of a global solution of the partial differential equation (5.10), and on system (5.4). The entire analysis so far has assumed that $(5.4)$ has a locally stable equilibrium at $\vec{x}=0$. If there are other locally stable equilibria it will be necessary to start solutions in the neighborhood of each equilibrium and the situation becomes complicated in a way we will discuss later. However, if the nolse terms are simple in their functional dependence on $\vec{x}$, and if the system globally has just the one equilibrium at $\vec{x}=0$, we expect that $v(\vec{x})$ will provide information on global stability.

Second, does $v(\vec{x})$ guarantee asymptotic stability or just stability? Here the fact that $(5.10)$ is derived from the stochastic problem comes into play. Basically, if the matrix $\left(a_{i j}\right)$ is positive and not just nonnegative, then at least locally $v$ guarantees asymptotic stabi1ity. Further, a look at the solution procedure detailed in Appendix C shows that as long as the solution works, 1.e., the solution curves move away from $\vec{x}=0$ to cover $\vec{x}$-space, the gradient of $v$ is nonzero. A nonzero gradient of $v$ together with a positive $\left(a_{1 f}\right)$ ensures asymptotic stability. The latter criteria appear to be satisfied in most examples of white noise models which have been used in the past (see the example presented next).

We have established that the Liapunov function $v(\vec{x})$ for the nonlinear deterministic model $\frac{d \vec{x}}{d t}=G(\vec{x})$ characterizes the temporal behavior of the stochastic model produced by adding white noise. A simflar result has long been known to be true for linear models (Lewontin 1969). If the sample paths of the stochastic model (5.7) are compared with the deterministic trajectories of (5.4), it is expected that for small 
Gaussian white noise the sample paths will be close to the deterministic trajectorles. In fact the sample paths will have a roughly Gaussian distribution around deterministic trajectories (Vent'sel and Friedif 1970), and the width of this distribution decreases with $\varepsilon$. All this suggests that our result is an expression of the fact that the stochastic process yields sample paths 'close' to determinlstic trafectories. The surprising thing about the result is that it relates a picture of certain extinction in the stochastic model with a picture of guaranteed stability in the deterministic model. The strength of the result is that it holds true for very general models, and is independent of the choice of stochastic calculus.

A final observation concerning $v(\vec{x})$ is relevant to May's (1974) discussion of stochastic models. Consider the neighborhood of the equilibrium $\vec{x}=\vec{t}$. For $|\vec{x}|$ very small the various terms in (5.10) may be expanded around equilibrium, and we find that

$$
v(\vec{x}) \simeq \vec{x}^{\prime} B \vec{x}
$$

where the matrix $B$ is the solution of the matrix equation

$$
B^{-1} G+G^{\prime} B^{-1}=-A \text {. }
$$

In (5.14), matrix $G$ is the Jacobian $(\partial \vec{G} / \partial \vec{x})$ evaluated at $\vec{x}=\overrightarrow{0}$, and $A$ is the matrix $\left(a_{1 j}\right)$ from (5.8). Equation (5.14) is just the well-known Liapunov matrix equation expressing nelghborhood stability of $\vec{x}=\overrightarrow{0}$, while (5.13) is a typical form for a Liapunov function around equilibrium (Hahn 1970). If we now assume that the extinction boundary $\Sigma$ is very close to the equilibrium $\vec{x}=0$, and in fact pick $\Sigma$ to be the ellipsold $\vec{y}^{\prime} B \vec{y},|\vec{y}| \ll 1$, then the approximate time over which the stochastic system stays within $\sum$ is, from (5.11), 


$$
T_{0} \simeq \exp \left[+\frac{\vec{y}^{\prime} B \vec{y}}{\varepsilon}\right]
$$

This expression (5.15) tells us that the populations will stay very close to equilibrium for a time $T_{0}$, and $T_{0}$ is increased if the value of the exponential can be increased. This provides a new perspective on May's (1974) argument that a ratio 1dentical to that in the exponent of (5.15) be made as large as possible to increase stochastic stability. To conclude this rather long section, we now present a short illustrative example, which is particularly relevant in the context of previous work on stochastic models.

\section{An Example}

Here we apply the method fust described to a popular class of ecological models. The deterministic models of this class have the form

$$
\frac{d n_{1}}{d t}=n_{1} F_{1}(\vec{n}), \quad 1=1, \ldots, m
$$

and possess an equilibrlum at $\vec{n}=\vec{n}$. Nolse is added to produce a stochastlc process $\vec{N}(t)$ as follows,

$$
\frac{d N_{1}}{d t}=N_{i} F_{1}(\vec{N})+\sqrt{\varepsilon} N_{1} Z_{1}(t), \quad 1=1, \ldots, m
$$

where $Z_{1}(t), i=1, \ldots, m$, are independent 'white noise processes' described by equation (5.6). The particular form of (5.17) is drawn from the analysis of fluctuations in carrying capacity by May (1974). The form (5.17) is interesting because it leads to a rather simple equation for the Llapunov function $v(\vec{x})$.

As discussed earlier and also in Appendix B, two forms of the 
diffusion equation (5.8) may be formulated for the density $f(\vec{n}, t)$ of the $\vec{N}(t)$ process, 1.e., equations of the form $L_{*} f(\vec{n}, t)=\frac{\partial}{\partial t} f(\vec{n}, t)$. We now change variables to a set $\vec{x}(t)$ and a corresponding density $f(\vec{x}, t)$, defined by

$$
\begin{gathered}
x_{1}=\ln \frac{n_{1}}{n_{1}^{t}}, 1=1=1, \ldots, m, \\
f(\vec{x}, t) d x_{1} \ldots d x_{m}=f(\vec{n}, t) d n_{1} \ldots d n_{m} .
\end{gathered}
$$

It is necessary to change variables after obtaining the diffusion equation for $f(\vec{n}, t)$ because the Ito calculus is not coordinate-invariant (Mortensen 1969). The use of (5.18) yields two diffusion equations of the type $L_{*} f(\vec{x}, t)=\frac{\partial}{\partial t} f(\vec{x}, t)$. If the methods of the previous subsection are applied, the following equation for the Liapunov function $v(\vec{x})$ is obtained

$$
\frac{1}{2} \sum_{1}\left(\frac{\partial v}{\partial x_{1}}\right)^{2}+\sum_{i} F_{1}(\vec{x}) \frac{\partial v}{\partial x_{1}}=0
$$

Note that the matrix $\left(a_{i j}\right)$ here is diagonal and positive-definite. (Compare (5.10) and the subsequent discussion of $v(\vec{x})$. )

The relative simplicity of (5.19) allows a solution for $v(\vec{x})$ to be written down directly for a rather special case of (5.16), the antisymmetric Lotka-Volterra model. The example is admittedly artificial, but is useful as an illustration. The model is defined in terms of (5.16) by the functions

$$
\begin{aligned}
F_{1}(\vec{n}) & =k_{1}-n_{1}-\sum c_{1 j} n_{j}, \\
c_{1 j} & =-c_{j 1}, \\
k_{1} & =1+\sum_{j} c_{1 j}, \\
n_{1}^{*} & =1, \quad{ }_{1, j}=1, \ldots, m, j \neq 1 .
\end{aligned}
$$


The solution of (5.19) in this case is just

$$
v(\vec{x})=\sum_{1} 2\left[\exp \left(x_{1}\right)-x_{1}-1\right]
$$

Note that $v(\vec{x})$ in $(5.20)$ is known to be a Llapunov function which guarantees global asymptotic stability about $\vec{x}=\delta$ for the deterministic model (5.20) (Alken and Lapidus 1974).

To apply (5.21) to the question of finding $\lambda_{0}$ for the stochastic problem (5.17), we define an absorbing boundary $\Sigma$. This is done, for example, by specifying a lower bound $s$ on every $x_{1}$. A population is then considered extinct if its value drops below exp(s). The boundary contributions indicated in (5.11) come from the hyperplanes which form $\Sigma$,

$$
\sum=\left\{\text { the hyperplanes } x_{1}=8, x_{j \neq 1}=0,1, j=1, \ldots, m\right\} \text {. }
$$

In particular the values of $v(\vec{x})$ which contribute to $\lambda_{0}$ come from points $\Sigma^{*}$ on $\Sigma$ where $\mathrm{v}(\vec{x})$ has 1 ts minimum value. We then have $\lambda_{0} \sim \exp$ $\left[-\frac{v\left(\Sigma^{*}\right)}{\varepsilon}\right]$. The two-species case of $(5.20)$ is analyzed in detail by Ludwig in DL 75.

This illustration concludes the discussion of our result, and we now go on to an examination of the role played by different Llapunov functions for the same deterministic model when analyzing stochastic stability.

\section{LIAPUNOV FUNCTIONS: GEOMETRY AND STABILITY}

\section{The Geometrical View of Stability}

Theoretical discussions of the dynamics of nonlinear population models have made widespread use of a graphical visualization of the stability properties of such models. Population sizes in an m-species 
model are visualized as a point on an m-dlmensional surface called a 'dynamical landscape'. The geometry of this landscape is thought of as representing the stability properties of the model: thus neighborhoods of stable equilibria are seen as valleys, equilibria are valley bottoms if stable and mountain tops if unstable (Slobodkin 1961, MacArthur 1970, Lewontin 1969, May 1974, Gilpin 1974, Holling 1973). We focus here on the assumption that a suitable dynamical landscape is in fact provided by the surface of a Liapunov function for the nonlinear model. We demonstrate that several assumptions about the relationship between the geometry of a Liapunov surface and the stabllity of the model are simply not true in general.

To fix ideas, suppose the deterministic model to be equation (5.4) and let $\phi(\vec{n})$ be some arbitrary Liapunov function describing the stability of (5.4) in some region $S$ around the equilibrium $\mathrm{n}^{*}$. The following assumptions about the Llapunov surface $y=\phi(\vec{n})$ are often made:

1. In a sufficiently small neighborhood of $\vec{n}^{*}$ the Liapunov surface $y=\phi(\vec{n})$ is a smooth cup or bowl.

2. In the entire region $S$ the geometrical features of the surface $y=\phi(\vec{n})$ provide a guile to the character of the vector field $\vec{G}(\vec{n})$ which defines the model (1.e., $\frac{d \vec{n}}{d t}=\vec{G}(\vec{n})$ ). For example, the slope of the Liapunov surface Indicates the 'speed' at which the system moves. This assumption is sometimes stated in a plcturesque way by describing the dynamics of the model as the dynamics of a particle 'rolling' on the surface $y=\phi(\overrightarrow{\mathrm{n}})$.

3. If noise is added to the deterministic model (for example, as in (5.7) ), the resulting stochastic process is seen as motion in a 
'potential field' or 'force field' described by the surface $y=\phi(\vec{n})$ plus superimposed random perturbations. The depths of the 'valleys' and the slopes of the 'valley sides' of the surface $y=\phi(\vec{n})$ are assumed to characterize stochastic stability. Thus deeper, steeper valleys indicate greater stability.

None of these three assumptions is true for general nonlinear models. We emphasize that some of these assumptions can in fact be proved for special classes of models such as linear models and gradient models (linear models are not considered here, although gradient models are). However, our arguments show that for general nonlinear models the ideas listed in 1,2 , and 3 above can be misleading and should not be used unless they can be proved.

The geometrical interpretation of a Liapunov surface, if it is mathematically justified, can be intuitively satisfying and conceptually rich. Without justification, however, the geometrical interpretation of a Liapunov surface can mislead because of two essential points about Liapunov stability theory (Hahn 1969). First, finding a Liapunov function proves that from any initial state the system eventually reaches equilibrium, but does not provide information on the actual trajectory along which the system moves. Second, Liapunov functions are not unique.

We now deal briefly with assumption 1 above, and then present an example which makes plain the problems with assumptions 2 and 3 . The picture of $\phi(\vec{n})$ as a 'bowl' or 'cup' near $\vec{n}^{*}$ rests on the Idea that $\phi(\vec{n})$ (belng a positive-definite, continuous, differentiable function) should have an isolated relative minimum at $\vec{n}^{*}$. This idea is incorrect, 
as recently demonstrated by Inselberg and Dula (1974). An actual Liapunov surface $y=\phi(\vec{n})$ may in fact be rapidly oscillating (or undulating) around $\vec{n}^{*}$, so long as it can be sandwiched between two smooth surfaces which increase monotonically with $\left|\vec{n}-\vec{n}^{*}\right|$. In such a case $\vec{n}^{*}$ is not an isolated relative minimum for $\phi(\vec{n})$, and thus $y=\phi(\vec{n})$ is not like a bowl even very close to $\vec{n}^{*}$. For an example see Inselberg and Dula (1974); as they point out, a more accurate picture of $y=\phi(\vec{n})$ near $\overrightarrow{\mathrm{n}}+$ is of a surface being 'squeezed' between two smooth 'cups' or 'bowls'.

\section{Geometrical Pitfalls: An Example}

The geometrical assumptions 2 and 3 just now stated involve the deterministic stability and the stochastic stability of nonlinear models. We illustrate the inadequacy of these assumptions by displaying Liapunov functions for the two-species symmetric Lotka-Volterra competition model. The model has the general form (5.16) discussed previously, and is written as

$$
\begin{aligned}
& \frac{d n_{i}}{d t}=n_{i}\left(1+\alpha-n_{i}-\alpha n_{j}\right), \\
& n_{i}^{*}=1 \\
& i, j=1,2, \quad i \neq j .
\end{aligned}
$$

Here $\alpha$ is the competition coefficient. Transforming variables as in (5.18) to $x_{i}=\ln n_{i}$, we get

$$
\begin{aligned}
& \frac{d x_{i}}{d t}=-\left[\exp \left(x_{i}\right)-1\right]-\alpha\left[\exp \left(x_{j}\right)-1\right], \\
& i, j=1,2, i \neq j .
\end{aligned}
$$

There are four different Liapunov functions known for this system: MacArthur's (1970) function, 


$$
\phi_{1}(\vec{x})=\left[\exp \left(x_{1}\right)-1\right]^{2}+2 \alpha\left[\exp \left(x_{1}\right)-1\right]\left[\exp \left(x_{2}\right)-1\right]+\left[\exp \left(x_{2}\right)-1\right]^{2}
$$

Tuljapurkar and Semura's (1975) function,

$$
\phi_{2}(\vec{x})=\exp \left(x_{1}\right)+\exp \left(x_{2}\right)-x_{1}-x_{2}-2 \text {. }
$$

Gilpin's (1974) function, defined as an integral over trafectories of the system $(5.23)$,

$$
\phi_{3}(\vec{x})=\int_{\vec{x}}^{\overrightarrow{0}} \frac{d \vec{x}}{d t} \cdot d \vec{x} .
$$

Ludwig's (DL 75) function, obtained by adding white noise to (5.22)

exactly as specified by (5.17), and then solving (5.19) to get

$$
\phi_{4}(\vec{x})=v(\vec{x}) \text {. }
$$

Note that only $\phi_{4}(\vec{x})$ is known to provide information on stochastic stability. Contours $\phi(\vec{x})=$ constant for these functions are displayed on the $\left(x_{1}, x_{2}\right)$ plane in Figures 6 through 9 for $i=1, \ldots, 4$. For the contours we use $\alpha=0.7$ (mainly because $\phi_{4}(\vec{x})$ could just be taken from DL 75). The function $\phi_{3}(\vec{x})$ was computed by an actual integral over trajectories as discussed by Gilpin (1974).

A comparison of Figures 6 through 9 shows that the contours $\phi_{i}(\vec{x})$, $i=1, \ldots, 4$, are in fact qualitatively different. The actual magnitudes of $\phi_{1}(\vec{x})$ are unimportant, but the shapes of the contours and the gradients of the functions are different. Visualized in three dimensions, the four 'valleys' have rather different topographies. Such differences exist for all nonzero $\alpha$ (it is easy to compute at least $\phi_{1}$ and $\phi_{2}$ for various $\alpha)$.

Two conclusions follow. First, in view of the differences in Figures 6 to 9, an arbitrary Liapunov surface does not provide a 


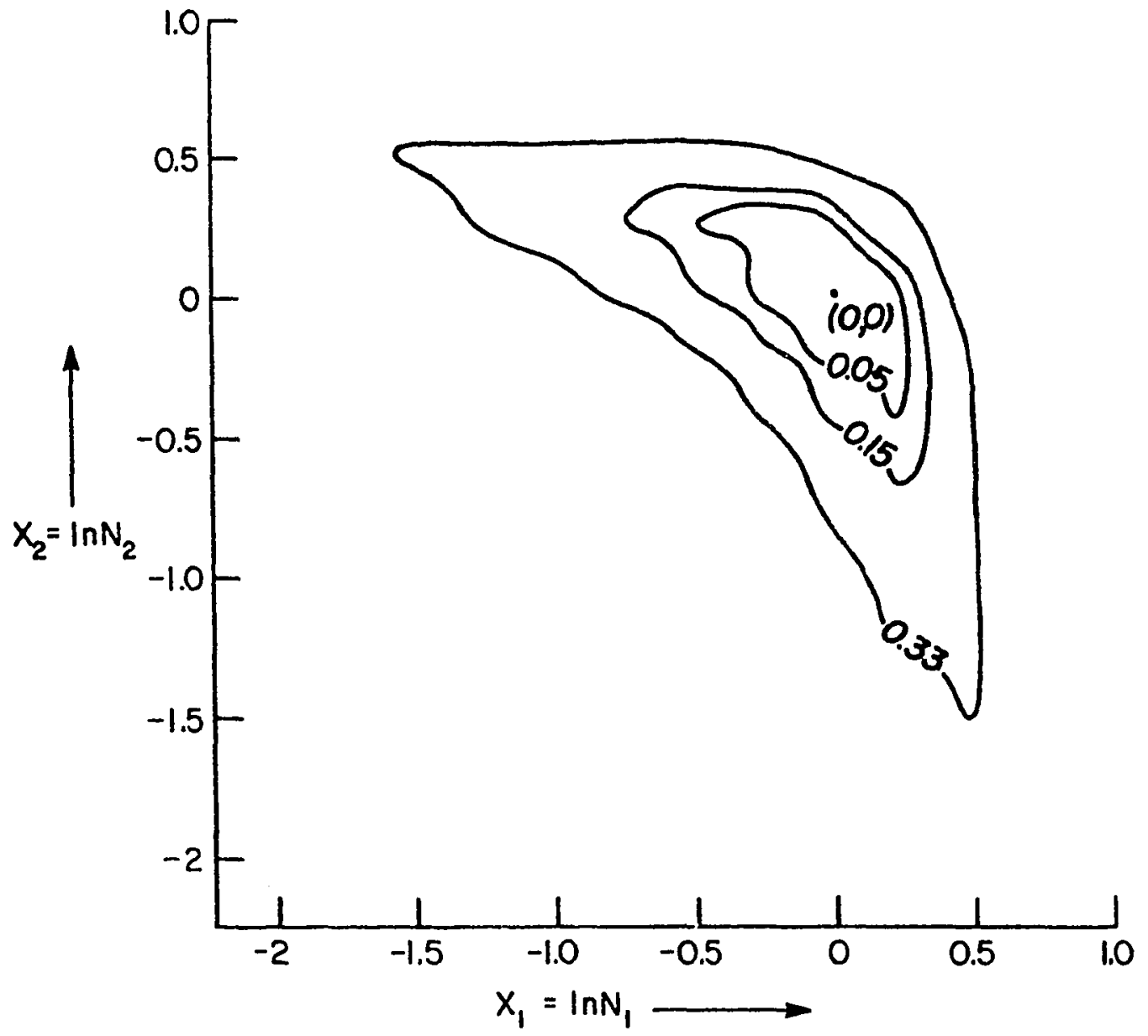

Figure 6. Contours of constant value for $\phi_{1}$. 


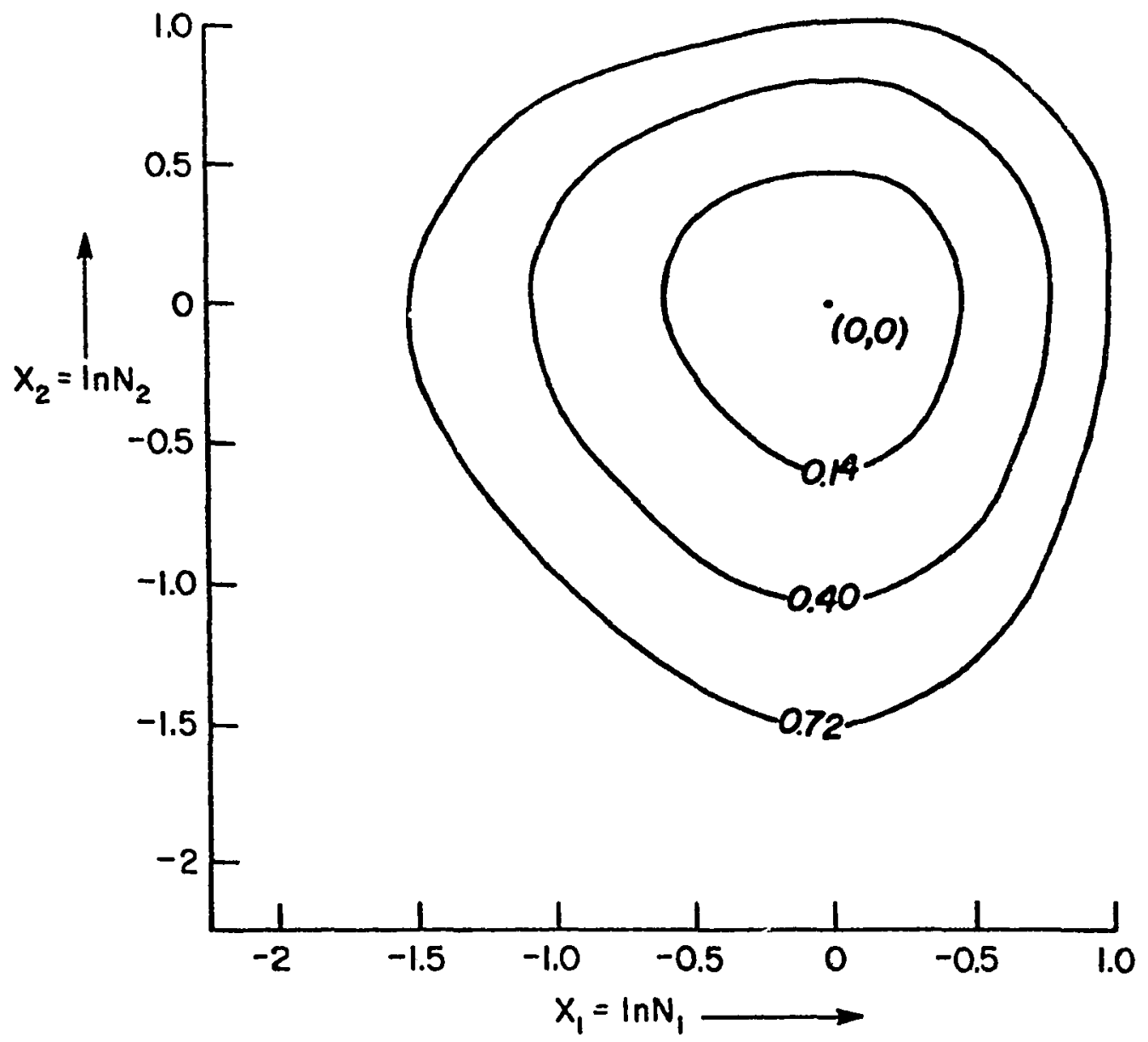

Figure 7. Contours of constant value for $\phi_{2}$. 


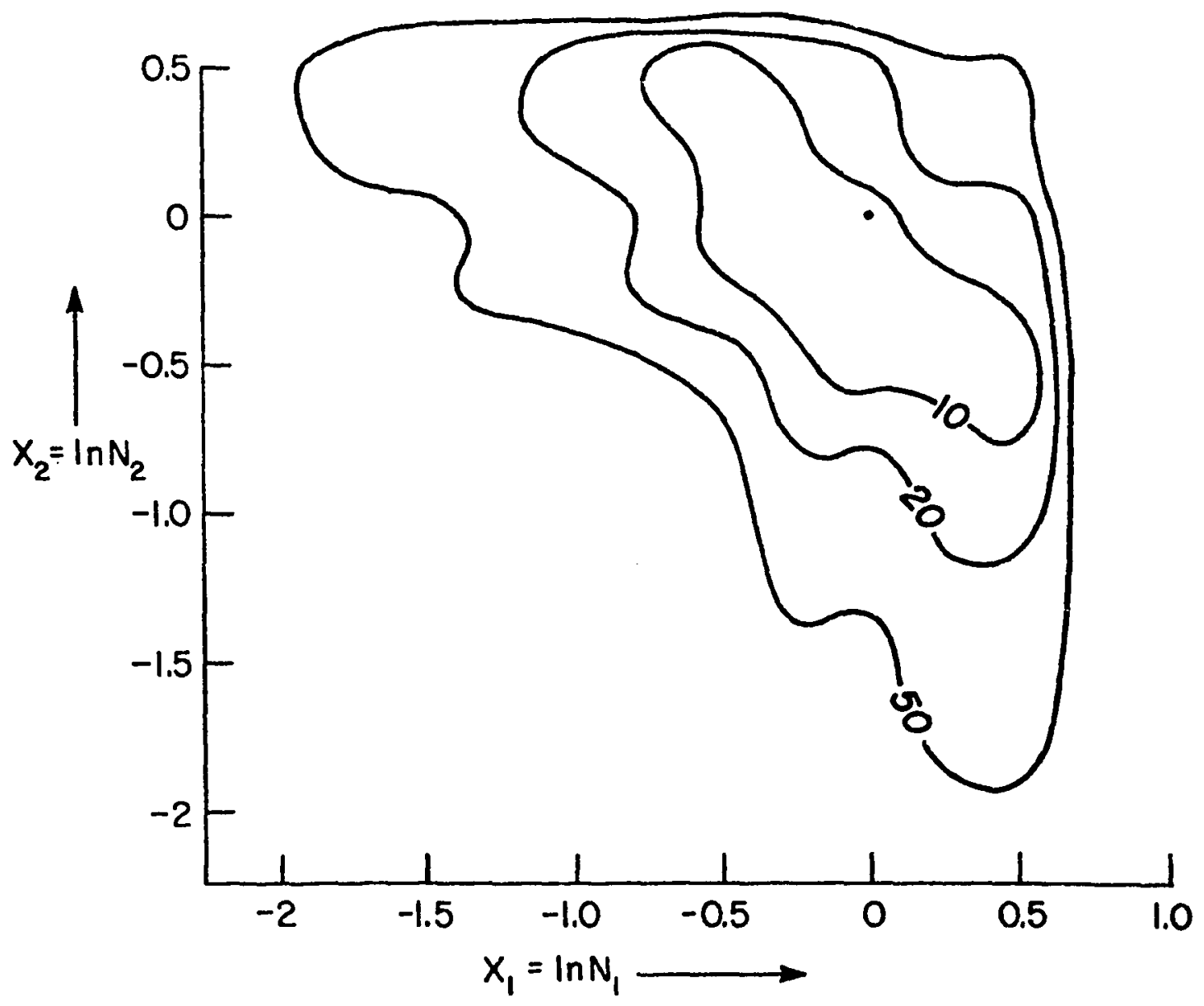

Figure 8. Contours of constant value for $\phi_{3}$. 


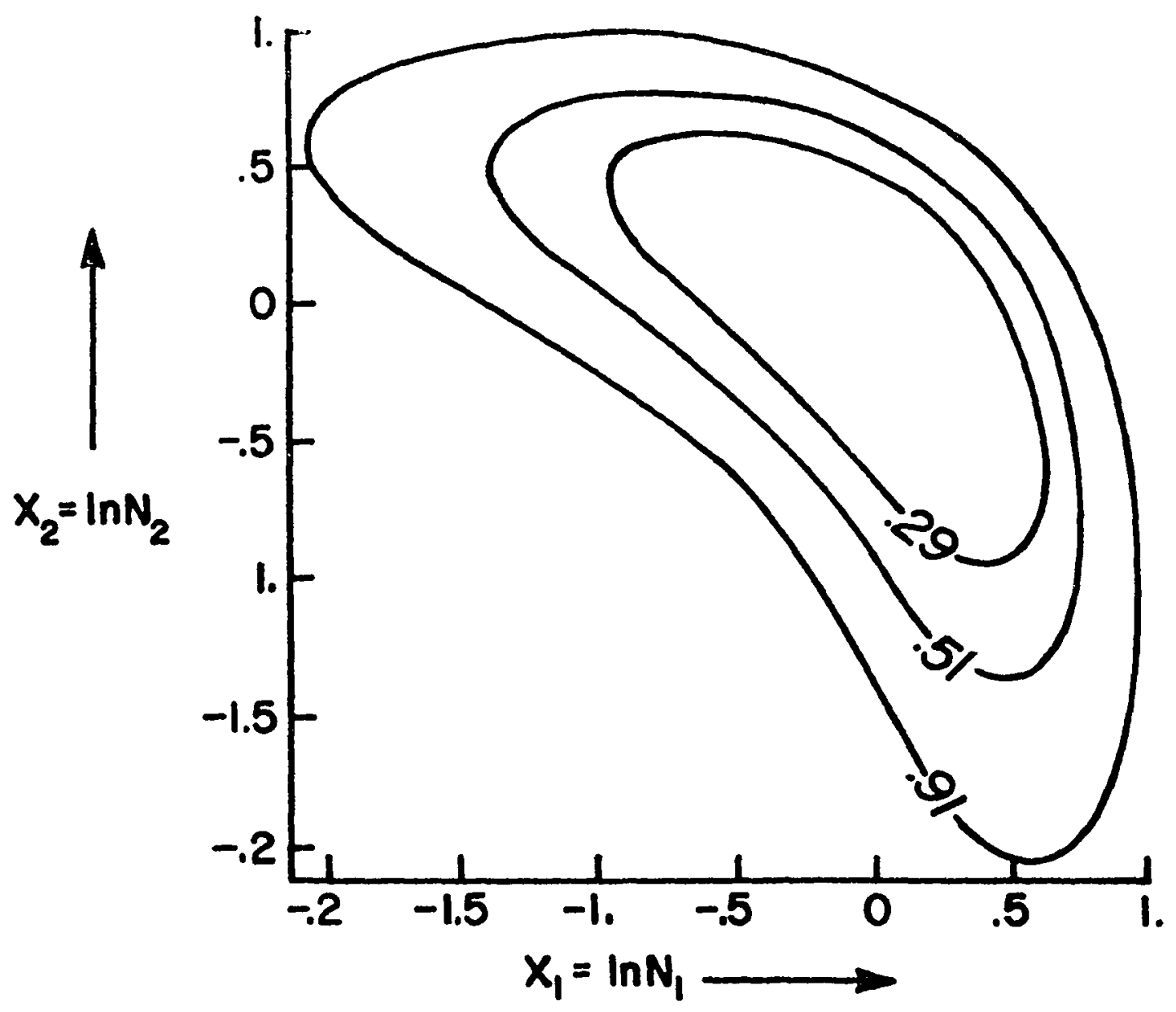

Figure 9. Contours of constant value for $\phi_{4}$. 
geometrical characterization (as in assumpion 2) of the deterministic stability of a nonlinear model. Second, only $\phi_{4}(\vec{x})$ satisfies (5.19) (the other functions do not), and so only $\phi_{4}(\vec{x})$ (or in general $v(\vec{x})$ ) provides information on stochastic stability. It is important to note that $v(x)$ is very specifically related to the stochastic extinction picture we have discussed, and does not in general provide any other geometrical information about stochastic stability.

We now briefly consider a special class of systems where assumptions 2 and 3 are in fact valid: gradient models. A gradient model has the general form

$$
\frac{d \vec{n}}{d t}=-\vec{\nabla} \psi(\vec{n})=\vec{G}(\vec{n})
$$

The equilibrla of $(5.28)$ are the minima of the function $\psi(\vec{n})$, which serves as a Liapunov function for $(5.28)$. Clearly the geometry of $\psi(\vec{n})$ does accurately reflect the dynamics of (5.28). Additionally if white noise is added to $(5.28)$ as indicated earlier, the diffusion equation (5.8) has the stationary solution (DL 75) $\mathrm{f}^{*}(\vec{x})=\exp \left[-\frac{2 \psi(\vec{x})}{\varepsilon}\right]$. Therefore $\psi(\vec{n})$ characterizes the stochastic model as well. This picture can even be extended to models where some purely rotational field $\vec{h}(\vec{n})$ is added to the gradient field $\vec{\nabla} \psi(\vec{n})$ (i.e., $\vec{h} \cdot \vec{\nabla} \psi=0$ ) (see Vent'sel and Friedlin 1970 for a discussion).

The results stated above for gradient models may be of limited use, as few ecological models appear to be gradient models. To sum up this section, geometrical assumptions about the nature of Liapunov surfaces for nonlinear population models are of doubtful valldity at best. They need mathematical justification if accurate conclusions about population models are to be drawn. We now turn to a general discussion of 
extinction and stability in ecological models.

\section{DISCUSSION}

The issues and assumptions involved in the study of temporal population fluctuations as represented by stochastic noise processes have been discussed. The specific case of white noise perturbations of nonlinear deterministic population models has been studied in detail. It has been shown that stochastic models involving white noise predict eventual extinction of one or more populations. Given an extinction boundary, i.e., population numbers below which specles are consldered to be extinct, an expected time to reach the extinction boundary, $\mathrm{T}_{0}$, can be defined. It has been shown that $\mathrm{T}_{0}$ is determined qualitatively by a Liapunov function for the original nonlinear deterministic model (for small perturbations and independent of the choice of stochastic calculus).

The feature of certain eventual extinction in stochastic models involving white noise is a consequence of the mathematical formulation of the models. Stochastic population models which predict that one or more populations will eventually reach absorbing states (1.e., go extinct) may generally be classtfied as nonergodtc models. Models invo1ving wl te noise are nonergodic, as are the different stochastic models cited in an earlier section. The structure of nonergodic models is such that no nontrivial equilibrium probability density exists, and so these models are charicterized by the 'extinction nightmare' remarked upon by Korostyshersky et. al. (1974).

In general the eventual extinction predicted by nonergodic models 
is mathematically rather than biologically significant. Consider the expected time to extinction, $\mathrm{T}_{0}$, for stochastic models involving white noise. As the extinction boundary $\Sigma$ is moved further away from equilibrium, the magnitude of $T_{0}$ increases rapldly (for an illustration, see the example at the end of the third section). Therefore, the mathematical behavior of nonergodic models does not imply the rapid disappearance of populations. From a biological viewpoint, nonergodic models are of course useful in situations where extinction is in some sense a real possibility, but can also be more generally useful, as we point out below.

Let us again consider specifically models involving white noise. If a model of this type is used to represent an isolated ecological community, or an isolated part of some larger community, then extinction may be a real biological possibility. An extinction boundary for an isolated community can be defined in practice as some finite population size below which a population is efther unobservable or plays no significant role in the interactions between populations in the community. In such a situation, the quantity $\mathrm{T}_{0}$ becomes relevant as a measure of time to extinction. However, the actual value of $T_{0}$ is probably more useful as a relative than an absolute measure of the time to extinction, in vlew of the many simplifying assumptions specified previously.

More generally, $\mathrm{T}_{0}$ is perhaps most accurately interpreted as a relative measure of the ability of populations to stay within a specified range of population numbers when subjected to completely random perturbations. This interpretation enables us to illustrate that $T_{0}$ 
may be useful in the extraction of more general blological implications from models. As a first example, we note that $T_{0}$ will depend on the values of the various parameters that go into the stochastic model. It would therefore be feasible to study $T_{0}$ as a function of the parameters in order to gain insight into the regions of parameter space where stochastic stability is greatest. Such insight is relevant to fleld tests of theory (Holling 1973) and to the application of general theory to specific biological questions (such as species packing; see Abrams 1976). Another example follows from the observation that the extinction boundary $\Sigma$ may actually be a boundary between two domains of attraction or between the attracting neighborhoods of two equilibrla (Holling 1973). In the latter case, it might be feasible to start solutions in the neighborhood of both equilibria, and use these solutions to determine the expected time for the populations to move from one neighborhood to the other. In these examples $T_{0}$ is viewed as a relative measure of stochastic stability. In some ways this view of $\mathrm{T}_{0}$ is similar in spirit to Holling's (1973) concept of the 'resilience' of ecosystems.

As we pointed out previously following the discussion of boundary conditions, it is easy to construct models which have a nontrivial equilibrium distribution. Such models may generally be called ergodic models. In the general class of Markov models, ergodicity is usually achieved by making the model 'open' rather than 'closed', 1.e., populations can leave states which would have been absorbing in a nonergodic model. A straightforward example of an ergodic open Markov model is one where immigration and emlgration processes are included (Takeyama 1975, Bailey 1962). Even in ergodic models, there is usually a finite 
nonzero probability of extinction, but this probability is less than one. Many real ecosystems are open to immigration and emigration processes and may be more accurately modeled by ergodic models than nonergodic ones.

In ergodic models the value of the extinction probability is a measure of stochastic stability analogous to the expected time to extinction in nonergodic models. A rather general use of such measures of stochastic stability is suggested by the work of Mountford (1973): It may be possible to study the general strategies which populations adopt in order to minimize the probability of extinction (or the time to extinction). The elucidation of the strategy of populations has been considered as one meaningful approach to ecology (Hughes and Gilbert 1970, Slobodkin 1969). The maximization of stochastic stability may be a criterion which governs some strategies of population behavior. Mountford (1973) has used such a criterion in the framework of a branching-process model to examine the significance of clutch size in birds. It is of course necessary to caution that teleological criteria such as this may be of limited validity.

Both ergodic and nonergodic models very often start from an equilibrium picture of the deterministic world. In these models attention is focused on equilibrium and the neighborhood of equilibrium, and the measures of stochastic stability which emerge from the models are concerned largely with departures from equilibrium. A rather different view of stochastic stability is concerned with the historical timepaths followed by the components of an ecosystem. Botkin and Sobel (1974) present a discussion of this historical viewpoint together with 
examples. Both the equilibrium-centered and the historical viewpoints can clearly be relevant to ecology. It may well be that the view of stochastic stability discussed in this chapter will need to be fused with the other approaches we have outlined before a valid description of complex ecosystems is posstble. 
CHAPTER VI

KINETIC EQUATIONS

INTRODUCTION

This chapter is concerned with Mori's (1965a, b) kinetic equation approach to the dynamics of many-particle systems. The theory presented here will be employed to study the dynamics of one-dimensional Heisenberg magnets in the next chapter. As we have explained in Chapter I, the kinetic equation approach is one realization of a general way of studying the stochastic behavior of dynamical systems. The intuitive basis of the kinetic equation approach lies in a consideration of time scales very much akin to our analysis of ecological time scales in Chapter V. The mathematical formalism of the kinetic equation approach has been successfully applied to the study of equilibrium and nonequilibrium dynamics in physical systems (Berne and Harp 1970, Akcasu and Duderstadt 1969, Mori 1973, Keyes and Oppenheim 1973), and is currently being applied to non-Hamiltonian systems such as Markov processes (Schnelder 1976).

In the next section we summarize the basic results of the Mori theory of kinetic equations. The third and final section of this chapter considers the systematic inclusion of higher-order derivatives in the Mori equation, and presents a proof of the equivalence of this lat-

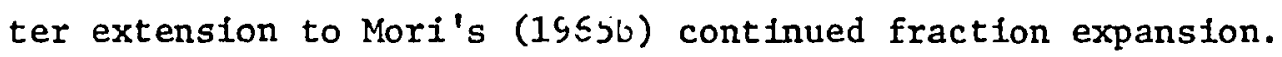


MORI'S THEORY

We consider a Hamiltonian system which is described by a dynamical variable $A(t)$ (here A may be a scalar or a column vector). The quantity $A$ is defined as the deviation from its equilibrium value (i.e., $A-\langle A\rangle$, where angular brackets indicate an average over an equilibrium ensemble). If the system Hamiltonian is $H$, the equation of motion for $A(t)$ is

$$
\frac{\mathrm{dA}(\mathrm{t})}{\mathrm{dt}}=\operatorname{ILA}(\mathrm{t})
$$

where in the classical case

$$
\operatorname{ILA}(t)=\{A(t), H\},
$$

and in the quantum case

$$
\operatorname{iLA}(t)=\frac{1}{1 / K}[A(t), H]
$$

with curly brackets indicating a Poisson bracket and square brackets a commutator. We define a scalar product of two variables $A$ and $B$ within which the Liouville operator $L$ is Hermitian,

$$
\left(A,[L B]^{*}\right)=\left(L A, B^{*}\right) \text {, }
$$

where $B^{*}$ is the Hermitian conjugate of $B$. Next we define a Hermitian projection operator $P$ which projects onto the initial value $A(t=0)=A$,

$$
P G=\frac{\left(G, A^{*}\right)}{\left(A, A^{*}\right)} A \text {. }
$$

(In the case where $A=\left(A_{i}, i=1, \ldots, m\right)$ the scalar product $\left(A, B^{*}\right)$ is the matrix with elements $\left(A_{i}, B_{j}^{*}\right)$ ).

Mori (1965a) has shown that the equation of motion (6.1) may be exactly reformulated as the kinetic equation 


$$
\frac{d A(t)}{d t}=1 w A(t)-\int_{0}^{t} \phi(s) A(t-s) d s+f(t)
$$

Here the frequency is defined as

$$
1 \omega=\frac{\left(\dot{A}, A^{*}\right)}{\left(A, A^{*}\right)},
$$

the random force is

$$
f(t)=e^{t(1-P) i L}(1-P) i L A,
$$

and the memory function is

$$
\phi(t)=\frac{\left(f(t), f^{*}\right)}{\left(A, A^{*}\right)} .
$$

The transformation from (6.1) to $(6.6)$ is exact for any choice of scalar product in $(6.4)$. The random force $f(t)$ is always orthogonal to $A$.

In many physical problems the following choice of scalar product is most useful, and we shall make this choice: classical,

$$
\left(A, B^{*}\right)=\left\langle A B^{*}\right\rangle
$$

quantum,

$$
\left(A, B^{*}\right)=\frac{1}{B} \int_{0}^{\beta} d \lambda\left\langle\exp (\lambda H) A \exp (-\lambda H) B^{*}\right\rangle,
$$

where the angular brackets indicate an average over the canonical ensemble $\exp (-\beta H) / \operatorname{Tr}[\exp (-\beta H)]$. The choice $(6.10),(6.11)$ is particularly useful in the regime where the instantaneous nonequilibrtum ensemble depends linearly on $A(t)$, in which case the nonequilibrium ensemble average of $f(t)$ is zero (Mor1 1965a, Nordholm and Zwanzig 1975) .

The relaxation function $R_{0}(t)$ is defined as 


$$
R_{0}(t)=\frac{\left(A(t), A^{*}\right)}{\left(A, A^{*}\right)}, R_{0}(0)=1,
$$

and from (6.6) obeys the equation

$$
\frac{d R_{0}(t)}{d t}=i \omega R_{0}(t)-\int_{0}^{t} \phi(s) R_{0}(t-s) d s .
$$

The Laplace transform of $(6.13)$ is of interest. Let

$$
A(z)=\int_{0}^{\infty} A(t) e^{-z t} d t
$$

be the Laplace transform. Then we have

$$
R_{0}(z)=\frac{1}{z-i \omega+\phi(z)} .
$$

The fourier transform of $R_{0}(t)$ is given by

$$
\begin{aligned}
R_{0}(\omega) & =\frac{1}{2 \pi} \int_{-\infty}^{\infty} R_{0}(t) e^{-i \omega t} d t, \\
& =\frac{1}{\pi} \operatorname{Re} R_{0}(z=i \omega),
\end{aligned}
$$

and the moments of this transform are defined by

$$
\left\langle\omega^{n}\right\rangle=\int_{-\infty}^{\infty} \omega^{n} R_{o}(\omega) d \omega .
$$

The significance of $R_{0}(t)$ and its moments lies in the fact that the experimentally measured response of a system to an external probe can be expressed directly in terms of $R_{0}(t)$. A knowledge of $R_{0}(t)$ is in a sense a description of the dynamics of the system.

Mori (1965b) has extended the above formalism in order to explore the time development of the random force $f(t)$. Let us write $f_{0}(t)=$ $A(t)$, and $f_{1}(t)=f(t)$. Now we define a projection operator $P_{1}$ which projects onto $E_{1}(0)=f(t=0)$, and operate on $\dot{f}_{1}(0)$ with $P_{1}$. The part of $\dot{\mathrm{f}}_{1}(0)$ orthogonal to $\mathrm{f}_{1}(0)=\mathrm{f}_{1}$ is called $\mathrm{f}_{2}$, and the process is 
continued to obtain a sequence of functions $f_{j}(t)$. Let $P_{j}$ be the projection operator projecting onto $f_{j}$, and define the operators

$$
L_{j}=\left(1-P_{j-1}\right) L_{j-1}, L_{o}=L, j \geq 1 \text {. }
$$

The sequence $f_{j}$ is then defined by

$$
\begin{aligned}
f_{j} & =i L_{j} f_{j-1}=\left\{\prod_{k=0}^{j-1}\left(1-P_{k}\right)\right\} i_{j-1} \\
& =\left\{1-\sum_{k=0}^{j-1} P_{k}\right\} i L f_{j-1}, j \geq 1, \\
f_{j}(t) & =e^{i L_{j} t} f_{j}, j \geq 0 .
\end{aligned}
$$

We have a corresponding sequence of relaxation functions,

$$
R_{j}(t)=\frac{\left(f_{j}(t), f_{j}^{*}\right)}{\left(f_{j}, f_{j}^{*}\right)} .
$$

Define in addition the quantities

$$
\begin{aligned}
& i \omega_{j}=\frac{\left(\dot{f}_{j}, f_{j}^{*}\right)}{\left(f_{j}, f_{j}^{*}\right)}, \\
& \delta_{f}=\frac{\left(f_{j}, f_{j}^{*}\right)}{\left(f_{j-1}, f_{j-1}^{*}\right)} .
\end{aligned}
$$

From (6.18) through (6.21) we obtain a heirarchy of equations

$$
R_{j}(z)=\frac{1}{z-1 \omega_{j}+\delta_{j+1}^{R} j+1}(z) \text {, }
$$

which may be proliferated into the continued fraction expansion 


$$
R_{0}(z)=\frac{1}{z-1 \omega_{0}+\frac{1}{z-1 \omega_{1}+\frac{\delta_{2}}{z-1 \omega_{2}+\ldots}}} .
$$

In (6.23) we have set $\omega_{0}=\omega$ from (6.7). The following useful general relation between the quantities in $(6.21)$ and (6.22) is worth noting,

$$
\left\langle\omega^{n}\right\rangle_{j}=\left(\omega_{j}-\omega_{o}\right)\left\langle\omega^{n-1}\right\rangle_{j}+\delta_{j+1} \sum_{k=2}^{n}\left\langle\omega^{n-k}\right\rangle_{j}\left\langle\omega^{k-2}\right\rangle_{j+1},
$$

where $\left\langle\omega^{n}\right\rangle_{j}$ are moments of $R_{j}(\omega)$ defined as in (6.17)

\section{EXTENDED DERIVATIVES}

We now set out in a general form the extension of Mort's theory by the systematic inclusion of higher derivatives of $A(t)$. For simplicity we take $A$ to be a scalar in this section, but note that the extension to a vector A can be straightforwardly obtained by the use of tensor products. Consider the set $A^{(j)}$ defined as

$$
A^{(j)}(t)=\frac{d^{j} A(t)}{d t^{j}}, A^{(0)}=A, j=0,1, \ldots
$$

We define the quantity $B_{0}=A$, and the projection operator $Q_{0}$ which projects onto $B_{0}$. Then define $B_{1}=\left(1-Q_{0}\right) A^{(1)}$, let $Q_{1}$ project on $B_{1}$, and obtain $B_{2}=\left(1-Q_{0}-Q_{1}\right) A^{(2)}$. In this fashion we obtain the set $B_{j}$, $j=0,1, \ldots$, using projection operators

$$
Q_{j} G=\frac{\left(G, B_{j}^{*}\right)}{\left(B_{j}, B_{j}^{*}\right)} B_{j} .
$$

The set $B_{j}$ is orthogonal and is defined generally by

$$
B_{j}=\left[1-\sum_{k=0}^{1-1} Q_{k}\right] A(j)
$$


We will use the orthogonal set $B_{j}(t)=\exp (1 L t) B_{j}$ as variables in the Mori kinetic equation.

First we prove some general properties of the varfables $B_{f}$. From (6.27) any $B_{j}$ can be written as

$$
B_{j}=A^{(j)}-\sum_{k=0}^{j-1} a_{j k} B_{k},
$$

where

$$
a_{j k}=\frac{\left.(A), B_{k}^{*}\right)}{\left(B_{k}, B_{k}^{*}\right)} \text {. }
$$

Using (6.28) we can write

$$
\dot{B}_{j}=I L B_{j}=A(j+1)-\sum_{k=0}^{j-1} a_{j k} \dot{B}_{k} \cdot
$$

From (6.28) and (6.30) it follows that for any $j$ :

1. $A^{(j)}$ is a linear combination of the set $\left\{B_{k}, k=0,1, \ldots, j\right\}$;

2. $\dot{B}_{j}$ is a linear combination of the set $\left\{B_{k}, k=0,1, \ldots, j+1\right\}$. Additionally we see that

$$
\begin{aligned}
\left(1-\sum_{k=0}^{j} Q_{k}\right) I L B_{j} & =\left(1-\sum_{k=0}^{j} Q_{k}\right) A^{(j+1)}, \\
& =B_{j+1},
\end{aligned}
$$

where we have used $(6.30)$ and property 2 above. In view of (6.31) we could just as well have generated the set $B_{j}, j=0,1, \ldots$ by using $(6.31)$ sequentially instead of (6.27). Now compare (6.31) with the generating equations (6.19) for the quantities $f_{j}$ in Mori's theory in the previous section. It is clear that the two are identical developments of an orthogonal set, and so we may make the identifications 


$$
\begin{aligned}
& P_{j}=Q_{j}, j=0,1, \ldots \\
& B_{j}=f_{j}, j=0,1, \ldots
\end{aligned}
$$

Note however that $f_{j}(t) \neq B_{j}(t)$ since the propagator for $f_{j}(t)$ is $\left(1-\sum_{k=0}^{j-1} P\right) \perp L$, while the propagator for $B_{j}(t)$ is just the Liouville operator IL.

We now turn to the extension of Mori theory. We work with derivatives extending out to nth order, $i$.e., with the set $A^{(j)}, j=1, \ldots, n$ or the equivalent set $B_{j}, j=1, \ldots, n$. Form the column vector

$$
B(t)=\left|\begin{array}{c}
B_{0}(t) \\
B_{1}(t) \\
\cdot \\
\cdot \\
\cdot \\
B_{n}(t)
\end{array}\right| \text {, }
$$

and use $B(t)$ as the dynamical variable In Mori's equation (6.6). We obtain the following kinetic equation

$$
\frac{d B}{d t}=i \omega B(t)-\int_{0}^{t} \Phi(s) B(t-s) d s+F(t) .
$$

The frequency matrix, memory function matrix and random force are found using (6.7) through (6.9) and (6.28) through (6.32) to be as follows.

First, the frequency matrix reduces to the form

$$
i \omega=\left(\begin{array}{ccccccc}
i \omega_{0} & 1 & 0 & 0 & 0 & \cdots & 0 \\
-\delta_{1} & i \omega_{1} & 1 & 0 & 0 & \ldots & 0 \\
0 & -\delta_{2} & i \omega_{2} & 1 & 0 & \cdots & 0 \\
& & & \cdots & & & \\
0 & 0 & \cdots & 0 & -\delta_{n-1} & 1 \omega_{n-1} & 1 \\
0 & 0 & \cdots & 0 & 0 & -\delta_{n} & 1 \omega_{n}
\end{array}\right) \text {, }
$$


where we have used the definitions given in (6.21). Second, the memory function matrix has the simple form

$$
\Phi(t)=\left(\begin{array}{ccccc}
0 & 0 & \cdots & \cdots & 0 \\
0 & 0 & 0 & \cdots & 0 \\
& \cdots & & 0 & 0 \\
0 & \cdots & \cdots & 0 & 0 \\
0 & \cdots & \cdots & 0 & \phi(t)
\end{array} \mid,\right.
$$

which results from the equally simple form of the random force,

$$
F(t)=\left(\begin{array}{c}
0 \\
0 \\
\cdot \\
\cdot \\
\cdot \\
0 \\
f_{n+1}(t)
\end{array}\right) \text {. }
$$

In (6.37) the function $f_{n+1}(t)$ is of course the $n$th component of $F(t)$, but the subscript $(n+1)$ is used to identify $F(t)$ with the functions defined by equation (6.19) of Mori theory, 1.e., we have

$$
f_{n+1}(t)=\exp \left\{\left[1-\sum_{j=0}^{n} P_{j}\right] i t L\right\}\left[1-\sum_{j=0}^{n} P_{j}\right] i L B_{n} .
$$

The single nonzero component of the memory function is then given by

$$
\phi(t)=\frac{\left(f_{n+1}(t), f_{n+1}^{*}\right)}{\left(B_{n}, B_{n}^{*}\right)}
$$

The proof of (6.35) through (6.39) is straightforward and utilizes extensively the properties in (6.28) through (6.32). A typical element of the frequency matrix in $(6.34)$ before reduction is 


$$
i \omega_{i j}=\frac{\left(\dot{B}_{1}, B_{j}^{*}\right)}{\left(B_{j}, B_{j}^{*}\right)} .
$$

Clearly from (6.32) and $(6.21), i w_{i 1}=1 w_{i}$. We now illustrate the reduction of (6.40) when $j<i$. Using (6.31) we proceed as follows:

$$
\begin{aligned}
\left(\dot{B}_{i}, B_{j}^{*}\right) & =-\left(B_{i},\left[\operatorname{iLb}_{j}\right]^{*}\right) \\
& =-\left(B_{i},\left[\left(1-\sum_{k=0} P_{k}\right) i L B_{j}\right]^{*}\right) \\
& =-\left(B_{i}, B_{j+1}^{*}\right) \\
& =-\delta_{i, j+1}\left(B_{i}, B_{i}^{*}\right) .
\end{aligned}
$$

In the above we have used the Hermitian property of $L$ anc $P_{j}$ A general application of the method illustrated in (6.41) leads to (6.35).

The simple form of $F(t)$ in (6.37) follows if we inspect the general expression for $F(t=0)$ which results on applying (6.8) to (6.34),

$$
F=(1-P) \perp L B \text {. }
$$

Here $\mathrm{P}$ is a projection operator onto the subspace spanned by the components of $B$ and is precisely the same as $\sum_{k=1}^{n} Q_{k}=\sum_{k=1}^{n} P_{k}$. From property (6.30) we see that the only component of ILB which is not in this subspace is iLB $_{n}$, and this leads directly to (6.37) and thence to $(6.38)$, (6.36).

The simple form of (6.34) through (6.39) makes the method of extended derivatives an appealing one to use. We now prove that in fact this method leads to precisely the same relaxation function $R_{0}(t)$ as Mori's continued fraction method. To show this we first apply (6.15) to (6.34) and obtain the following matrix of relaxation functions,

$$
R(z)=\frac{1}{z I-i \omega+\Phi(z)},
$$


where I is the unit matrix, the Laplace transform is as defined in (6.14), and the matrix $R(z)$ has elements

$$
R_{i j}(z)=\frac{\left(B_{i}(z), B_{j}^{*}\right)}{\left(B_{j}, B_{j}^{*}\right)} .
$$

In order to extract $R_{0}(z)=R_{00}(z)$ we need to invert the matrix (6.42) and extract the $(0,0)$ element of the inverted matrix. This element will have the form of a ratio of two polynomials in $z$,

$$
R_{0}(z)=\frac{G_{n}(z)}{F_{n}(z)},
$$

where $G_{n}$ is of order $(n-1)$ and $F_{n}$ of order $n$ in 2 . From the usual formula for inverse matrices we have that $F(z)$ is the determinant

$$
\begin{aligned}
F_{n}(z) & =|z I-i \omega+\Phi(z)|, \\
& =\left|\begin{array}{cccccc}
z-i \omega_{0} & -1 & 0 & \ldots & \ldots & 0 \\
\delta_{1} & z-i \omega_{1} & 0 & \ldots & \ldots & 0 \\
\cdots & \cdots & 0 & \delta_{n-1} & z-i \omega_{n-1} & -1 \\
0 & 0 & 0 & \delta_{n} & z-i \omega_{n}+\phi(z)
\end{array}\right| .
\end{aligned}
$$

If we were working with (n-1) derivatives we would have a polynomial $E_{n-1}(z)$ of exactly the same form as $(6.45)$ but $(n-1) \times(n-1)$. If we neglect the $z$-dependence of $\phi(z)$ we find from (6.45) that the $F_{j}(z)$, $j=1,2, \ldots$, obey the recursion formula

$$
\begin{aligned}
& F_{n}(z)=\left(z-i \omega_{0}\right) F_{n-1}(z)+\delta_{1} F_{n-2}(z), \\
& F_{1}(z)=z-i \omega_{0}+\phi(z) .
\end{aligned}
$$

Further $G_{n}(z)$ may be simply defined by the cofactor of the term $\left(z-1 \omega_{0}\right)$ in $(6.45)$. In the continued fraction theory. Mort (1965b) 
reduces the equation for $R_{0}(z)$ in (6.23), after termination at the $(n+1)$ th stage, to the identical ratio of polynomials as defined in (6.44) through (6.46). This completes our proof that termination of the continued fraction (6.23) at the $(n+1)$ th stage is formally exactly equivalent to including derivatives to order $\mathrm{n}$ in the Mori kinetic equation $(6.6)$. 
CHAPTER VII

COLLECTIVF MODES IN THE

FERROMAGNETIC HEISENBERG CHAIN

\section{INTRODUCTION}

The discovery in recent years of compounds which behave effective1y like one-dimensional (1-D) exchange-coupled magnets has stimulated theoretical interest in the dynamic properties of 1-D Heisenberg magnets (Steiner et. al. 1976, Hone and Richards 1974). In this chapter we seek to supplement existing theories of the isotropic Heisenberg Iinear chain by an examination of the collective modes, both propagating and diffuse, which exist in ferromagnetically coupled classical chains. We shall be concerned with the Hamiltonian

$$
H=-\frac{1}{2} \sum_{i, j} J_{i j} \vec{s}_{i} \cdot \vec{s}_{j}
$$

where the sums run over the $N$ lattice sites of a linear chain of spins with nearest-neighbor ferromagnetic $(J>0)$ interactions.

Our procedure is to use the spin variable $\vec{s}(\vec{k}, t)=\int_{1} \exp \left(i \vec{k} \cdot \vec{x}_{t}\right)$ $\vec{S}_{j}(t)$, and its time-derivatives of increasing order as dynamical variables in the generalized Langevin equation formalism due to Mor1 (1965a). We use Mori's criterion for the existence of good collective modes to isolate and display combinations of dynamical variables which constitute well-defined collective modes for the linear Heisenberg chain. The moments of the spin-relaxation function which are needed are estimated 
by the exact results available for the moments of the classical Heisenberg chain (Tomita and Mashiyama 1972).

We begin by using $S^{\alpha}(k, t)$ and $\dot{S}^{\alpha}(k, t)$ as dynamical varlables, and find collective modes described by the mode vectors $\dot{s}^{\alpha} \pm 1 \Omega_{0} s^{\alpha}$ where $\alpha=x, y$, or $z, \Omega_{0}=\left\langle\omega^{2}\right\rangle^{\frac{1}{2}}$ is the undamped frequency of the collective motion, and $\left\langle\omega^{2}\right\rangle$ is the second frequency moment of the spin relaxation function. At low temperatures we find that for wavevectors $k \ll k_{c}$ (where $k_{c}$ is the correlation length of Fisher's (1964) theory of the classical Heisenberg chain) the collective modes described by $\vec{S}(k, t)$ and $\vec{S}(k, t)$ break down in that $\Omega_{0}$ tends to zero while the damping of the modes becomes very large. We therefore extend our set of dynamical variables by including $\vec{G}(k, t)$ which is the part of $\ddot{\vec{S}}$ orthogonal to both $\vec{S}$ and $\vec{S}$. In the extended description three collective mode frequencies appear whose imaginary parts are $\pm 1 \Omega, 0$. For $k \gg k_{c}$ the extended picture reduces to the two-variable description in terms of $\vec{S}$ and $\vec{S}$, while for $k \ll k_{c}$ a diffusive mode, whose dynamics are essentially contained in $\vec{G}(k, t)$ as a mode vector, dominates the collective motion. Throughout our analysis the time-domain approximations involved in setting up good collective modes are made explicit.

The approach followed here of using higher derivatives in the Mori formalism has been suggested as an equivalent alternative to Mori's (1965b) continued fraction approximation by, for example, Kivelson and Ogan (1973) and Kim and Nelkin (1972). In fact there is a straightforward proof of the exact formal equivalence of the two approaches which we have presented in Chapter VI. An immediate consequence of this equivalence is that our analysis is closely related to the work of 
Tomita and Mashiyama $(1972,1974)$, Lovesey and Meserve (1972), Lovesey (1974), who use the continued fraction approximation. The time-domain analysis we make here focuses on the collective mode variables and their damped oscillatory behavior as described by equations of motion. The continued-fraction approximations cited above focus on the Iineshape function, and our results provide an interesting viewpoint on the continued-fraction method in terms of arguments concerning the separation of time-scales. Our principal objective in isolating collective modes is to motivate a physical picture of the propagating modes which are seen as excitations in linear magnets.

In the next section we discuss $\vec{s}(k, t)$ and its derivatives with emphasis on symmetry properties and linear dependence, and examine the choice of dynamical variables to be employed in Mori's generalized Langevin equation. Mori's equations have been summarized in the second section of Chapter VI and are not discussed here. Next we examine the two-variable case where $\vec{S}(k, t)$ and $\vec{S}(k, t)$ are employed, followed by the three-variable case where $\vec{G}(k, t)$ is added. The last section discusses our results in relation to earlier work and experiments.

\section{DYNAMICAL VARIABLES}

We first define our notation. The value of a dynamical variable $A(t)$ at time $t=0$ is written simply as $A=A(0)$. The Hermitian conjugate of $A(t)$ is written $A^{*}(t)$. The scalar product used is written $\left(A, B^{*}\right)$ and is defined for the classical and quantum cases in (6.10) and (6.11) respectively. Because of the absence of long-range order in the Iinear chain and the spin-rotational invariance of the Hamiltonian (7.1), 
scalar products of the form $\left(s^{\alpha}, s^{\beta}\right)$ are zero for $\alpha \neq \beta$. Therefore we treat the $x, y$ and $z$ components of $\vec{S}(k, t)$ as independent and will use the scalar variable $S(k, t)$ which represents any one of the three components.

The normalized spin relaxation function is defined as

$$
R_{0}(k, t)=\frac{\left(S(k, t), S(k)^{*}\right)}{\left(s(k), S(k)^{*}\right)},
$$

and its moments are defined as

$$
\left\langle\omega^{2 n}\right\rangle=\frac{\left(s^{(n)}(k), s^{(n)}(k)^{*}\right)}{\left(s(k), s(k)^{*}\right)},
$$

where $S^{(n)}(k)=(i L)^{n} S(k)$ is the nth time-derivative of $s(k, t)$ at $t=$ 0 ; L is the Liouville operator for the Hamiltonian (7.1). The exact moments (7.3) for $\mathrm{n}=1,2,3$ are given for the classical chain by Tomita and Mashiyama (1972). It is convenient to introduce the quantities

$$
\begin{aligned}
\delta_{1} & =\left\langle\omega^{2}\right\rangle \\
\delta_{1} \delta_{2} & =\left\langle\omega^{4}\right\rangle-\left\langle\omega^{2}\right\rangle^{2} \\
\delta_{1} \delta_{2} \delta_{3} & =\left\langle\omega^{6}\right\rangle-\frac{\left\langle\omega^{4}\right\rangle^{2}}{\left\langle\omega^{2}\right\rangle} \\
\delta_{1} \delta_{2} \delta_{3}\left(\delta_{3}+\delta_{4}\right) & =\left\langle\omega^{8}\right\rangle-\frac{2\left\langle\omega^{6}\right\rangle\left\langle\omega^{4}\right\rangle}{\left\langle\omega^{2}\right\rangle}-\frac{\left\langle\omega^{4}\right\rangle^{2}}{\left\langle\omega^{2}\right\rangle}
\end{aligned}
$$

The spin variable $S(k, t)$ has odd symetry under time-reversal, and the time derivatives $s^{(n)}(k, t)$ are even or odd under time reversal according as $n$ is odd or even. Therefore the scalar product $\left(S^{(\ell)}(k, t)\right.$, $\left.\mathrm{S}^{(\mathrm{m})}(k, t)\right)=0$ if $(\ell+m)$ is an odd integer. The fact that $\left(\dot{S}(k), s(k)^{*}\right)$ is zero contrasts with the ordered state of the Heisenberg magnet where $\left.\dot{s}^{+}(k), s^{-}(k)^{*}\right)$ is proportional to the magnetization $\left\langle s^{z}(k=0)\right\rangle$. Thus 
the orthogonality of $s(k, t)$ and $\dot{s}(k, t)$ reflects the absence of longrange order in the Heisenberg chain. This orthogonality suggests the use of $S(k, t)$ and $\dot{S}(k, t)$ as a minimal set of dynamical varlables for the linear chain.

We now introduce projection operators $P_{0}$ and $P_{1}$ which project an arbitrary variable onto the variables $s(k), \dot{s}(k)$ respectively. For example

$$
\mathrm{P}_{0} \mathrm{~A}=\frac{\left(\mathrm{A}, \mathrm{S}(\mathrm{k})^{*}\right)}{\left(\mathrm{S}(\mathrm{k}), \mathrm{S}(\mathrm{k})^{*}\right)} \mathrm{S}(\mathrm{k})
$$

Using $P_{0}, P_{1}$ we define the part of $\ddot{s}(k)$ orthogonal to $s(k)$ and $\dot{S}(k)$ as

$$
\begin{aligned}
G(k) & =\left(1-P_{0}-P_{1}\right) \ddot{s}(k) \\
& =\ddot{s}(k)+\delta_{1} S(k) .
\end{aligned}
$$

The variable $G(k, t)=\exp (i L t) G(k)$ is the next higher-order varlable to be used in the extended derivative scheme. To assess the importance of $G(k, t)$ which is the part of $\ddot{S}(k, t)$ orthogonal to $S(k, t)$ and $\dot{S}(k, t)$, we form the ratio

$$
\frac{\left(G(k, t), G(k, t)^{*}\right)}{\left(\ddot{S}(k, t), \ddot{S}(k, t)^{*}\right)}=\frac{\delta_{2}}{\delta_{1}+\delta_{2}} .
$$

From (7.7) we see that when $\frac{\delta_{1}}{\delta_{2}} \gg 1$ the magnitude of the orthogonal part of $\ddot{S}(k, t)$ is very small and so $\ddot{s}(k, t)$ is almost linearly dependent on $S(k, t)$ and $\dot{S}(k, t)$. However when $\frac{\delta_{1}}{\delta_{2}} \ll 1$ the magnitude of $G(k, t)$ is almost equal to that of $\ddot{s}(k, t)$, and so $\ddot{s}(k, t)$ is very nearly orthogonal to $S(k, t)$ and $\dot{S}(k, t)$. Therefore we qualitatively expect that $G(k, t)$ 
will become an important dynamical variable when $\frac{\delta_{1}}{\delta_{2}} \ll 1$. If we estimate $\frac{\delta_{1}}{\delta_{2}}$ from the values for the classical chain, the above reasoning suggests that $G(k, t)$ becomes important to the collective behavior for very sma1l wavevectors $k$.

We now proceed to an analysis of the Mori generallzed Langevin equation for the dynamical variables discussed above. We first conslder the variables $s(k), \dot{S}(k)$, and in accord with the comments made earlier find that the first two derivatives constitute good mode varfables only for high $k$. The subsequent analysis includes $G(k, t)$.

\section{TWO VARIABLE THEORY}

The description of collective motion in the Heisenberg chain in terms of $s(k, t)$ and $\dot{S}(k, t)$ is precisely analogous to the stochastic theory of the Brownian motion of a simple harmonic oscillator as discussed by Mori (1965a). We work with the orthogonal pair of variables

$$
I^{ \pm}=\dot{S}(k) \pm 1 \Omega_{0} S(k)
$$

where the frequency is defined by

$$
\Omega_{0}^{2}=\frac{\left(\dot{S}(k), \dot{S}(k)^{*}\right)}{\left(s(k), S(k)^{*}\right)}
$$

The random forces corresponding to $I^{ \pm}(t)$ in the generalized Langevin equations are identical and are given by

$$
f(t)=f^{ \pm}(t)=e^{t\left(1-P_{0}-P_{1}\right) i L}\left(\ddot{S}(k)+\Omega_{0}^{2} S(k)\right),
$$

where $P_{0}$ and $P_{1}$ are the projection operators defined in equation (7.5). We introduce the memory function 


$$
\phi(t)=\frac{\left(f(t), f^{*}\right)}{\left(\dot{S}(k), \dot{S}(k)^{*}\right)},
$$

which is related to the relaxation function of the random force

$$
\psi(t)=\frac{\left(f(t), f^{*}\right)}{\left(f, f^{*}\right)}=\frac{1}{\delta}{ }_{2} \phi(t) .
$$

The random force $f(t)$ is of course orthogonal to both $\mathrm{I}^{+}$and $\mathrm{I}^{-}$. Mori's exact equation of motion for $I^{ \pm}(t)$ may now be written as

$$
\begin{aligned}
\frac{d}{d t}\left(\begin{array}{c}
I^{+}(t) \\
I^{-}(t)
\end{array}\right)= & \left(\begin{array}{cc}
i \Omega_{0} & 0 \\
0 & -i \Omega_{0}
\end{array}\right)\left(\begin{array}{c}
I^{+}(t) \\
I^{-}(t)
\end{array}\right) \\
& -\int_{0}^{t} \frac{1}{2} \phi(s)\left(\begin{array}{ll}
1 & 1 \\
1 & 1
\end{array}\right)\left(\begin{array}{l}
I^{+}(t-s) \\
I^{-}(t-s)
\end{array}\right) d s \\
& +\left(\begin{array}{l}
f(t) \\
f(t)
\end{array}\right) .
\end{aligned}
$$

Equation (7.13) may be arrived at equivalently by starting with the exact Mori equation for $(s(k, t), \dot{S}(k, t))$ and diagonalizing the resulting frequency matrix.

Before proceeding to an examination of the collective behavior, we note that the random force relaxation function has the following short time expansion

$$
\psi(t)=1-\delta_{3} \frac{t^{2}}{2 !}+\delta_{3}\left(\delta_{3}+\delta_{4}\right) \frac{t^{4}}{4 !}-\ldots
$$

where the $\delta$ 's are defined in (7.4). The short time expansion of $\phi(t)$ is immediately obtained from (7.14) since $\phi(t)=\delta_{2} \psi(t)$ from (7.12).

Let $A(t)$ be the column vector with components $I^{ \pm}(t)$ and consider 
the relaxation function matrix $\left(A(t), A^{*}\right)\left(A, A^{*}\right)^{-1}=R(t)$. MorI's condition for $A(t)$ to represent good collective modes is that the relaxation function should satisfy the equation

$$
R(t+s)=R(t) R(s) \text {. }
$$

The general solurion to (7.15) has the form $R(t)=\exp [(1 \omega-\Gamma) t]$, where $\omega$ is a real frequency matrix and $\Gamma$ is a real damping matrix. Inspection of (7.13) shows that a solution of the latter form can be obtalned from the equation of motion only if the convolution integral in (7.13) can be decoupled into the product of some time-independent matrix with $A(t)$. Such a decoupling may be performed if the memory function $\phi(t)$ decays with a characteristic time $\tau_{1}$ which is much shorter than the characteristic decay time $\tau_{0}=\Gamma^{-1}$ of the relaxation function $R(t)$, 1.e.,

$$
\frac{1}{\Gamma} \gg \tau_{1}
$$

In the two-variable case the quantity $\tau_{1}$ is the characteristic decay time of the random force relaxation function and we may estimate this time as

$$
\tau_{1}=\int_{0}^{\infty} \psi(t) d t
$$

We now make the assumption that (7.16) is valid. The convolution integral in (7.13) may then be decoupled by writing

$$
\int_{0}^{t} \Phi(s) A(t-s) d s \approx\left[\int_{0}^{\infty} \Phi(s) e^{-i \omega s} d s \quad\right] A(t)
$$

where $\Phi(s)$ and $i \omega$ are the memory function matrix and the frequency matrix written out in full in (7.13). In order to actually compute the matrix in square brackets we need to specify $\phi(t)$ or equivalently $\psi(t)$. In view of (7.14) we now assume (i) the decay of $\psi(t)$ is monotonic, and elther (ii) $\tau_{1}$ is so short that the short-time expansion of $\psi(t)$ is good 
for all times $t$, or (iii) the higher $\delta$ 's such as $\delta_{4}$ in the coefficient of $t^{4}$ make a negligible contribution to the area under the curve of $\psi(t)$. With assumptions (i) and (ii) or (iii) we arrive at the Gaussian approximation

$$
\psi(t)=e^{-\frac{\delta_{3} t^{2}}{2}}
$$

for which

$$
\tau_{1}=\left(\frac{\pi}{2 \delta_{3}}\right)^{\frac{1}{2}}
$$

From the behavior of the exact $\delta_{3}$ for the classtcal Heisenberg magnet we find that $\delta_{3}$ is relatively insensitive to changes in wavevector $k$ at low temperatures, so at low temperatures the decay time $\tau_{1}$ in $(7.20)$ is also taken to be relatively insensitive to changes in $k$.

Inserting (7.19) in (7.18) we find that the damping matrix (in square brackets in $(7.18)$ ) reduces to

$$
\left(\begin{array}{cc}
\gamma+i \Delta \omega & \gamma-1 \Delta \omega \\
\gamma+i \Delta \omega & \gamma-1 \Delta \omega
\end{array}\right),
$$

where

$$
\begin{aligned}
& \gamma=\left(\frac{\pi}{32}\right)^{\frac{1}{2}} \delta_{2} \delta_{3}^{-\frac{1}{2}} \exp \left(-\frac{\delta_{1}}{2 \delta_{3}}\right), \\
& \Delta \omega=\left(2 \delta_{3}\right)^{-\frac{1}{2}} \exp \left(-\frac{\delta_{1}}{2 \delta_{3}}\right) \int_{0}^{x} e^{t^{2}} d t \text {, } \\
& x=\frac{\Omega_{0}}{\left(2 \delta_{3}\right)^{\frac{1}{2}}} \text {. }
\end{aligned}
$$

For the present we fgnore $\Delta \omega$ (this step is really legitimate only if $x \ll 1)$ since it does not materially affect the arguments immediately 
following. Then we may insert (7.21) without the $\Delta \omega^{\prime}$ 's Into (7.13) and diagonalize the resulting equation to get the collective mode equation

$$
\frac{d}{d t}\left(\begin{array}{c}
I_{1} \\
I_{2}
\end{array}\right)=\left(\begin{array}{cc}
i \Omega-\gamma & 0 \\
0 & -i \Omega-\gamma
\end{array}\right)\left(\begin{array}{l}
I_{1} \\
I_{2}
\end{array}\right) \text {, }
$$

where the mode frequency is given by

$$
\Omega^{2}=\Omega_{0}^{2}-\gamma^{2}
$$

and

$$
\begin{aligned}
I_{1}(t) & =(1+p) \dot{s}(k, t)+i \Omega_{0}(1-p) s(k, t), \\
I_{2}(t) & =(1-p) \dot{s}(k, t)-i \Omega_{0}(1+p) s(k, t), \\
p & =\frac{i \gamma}{\Omega_{0}+\Omega} .
\end{aligned}
$$

The collective modes described in (7.23) through (7.25) have been obtained under assumptions (7.16) and (7.19). If we now consider the damping of the collective modes $\gamma$ and use the exact moments at low temperatures for the classical chain in (7.22), we find that $\gamma$ increases as $k$ decreases for $k \ll k_{c}$. Additionally the ratio $\frac{\gamma}{\Omega_{0}}$ diverges as $k$ tends to zero. Therefore the modes described by (7.13) or (7.23) become heavily damped modes for $k \ll k_{c}$. If we consider now the time-scale criterion (7.16) with $\Gamma$ replaced by $\gamma$, we find that for $k \ll k_{c}$ as $k$ decreases to zero, $\frac{1}{\gamma}$ increases while $\tau_{1}$ stays almost constant. Therefore for small $k \ll k_{c}$ at low temperatures the assumption (7.16) about separation of time-scales breaks down.

We conclude that the damped propagating modes described by $I^{ \pm}(t)$ describe the collective motion in the Heisenberg chain at low temperatures except in the region of low wavevector $k$. The frequency, damping 
and frequency shift are given by $(7.24),(7.22)$. In order to describe consistently the collective modes for $k \ll k_{c}$ it is not possible to assume the separation of time scales expressed by (7.16). We therefore extend our set of dynamical variables to include $G(k, t)$ defined in $(7.6)$.

\section{THREE VARIABLE THEORY}

The breakdown of the assumption that the random force $f(t)$ of the two-variable theory fluctuates much more rapidly than $s(k, t)$ and $\dot{s}(k, t)$ suggests the inclusion of the slowly varying part of $f(t)$ In the set of dynamical variables. From the discussion of $G(k, t)$ following equation (7.7) it is seen that in the region $k \ll k_{c}, G(k, t)$ is nearly equal to $\ddot{s}(k, t)$ and is orthogonal to $s(k, t)$ and $\dot{s}(k, t)$. Therefore the inclusion of $G(k, t)$ as a dynamical variable should result in the inclusion of part of the orthogonal random force $f(t)$ of the two-variable theory.

We choose $S(k, t), \dot{S}(k, t)$ and $G(k, t)$ as components of a vector of dynamical variables. Diagonalizing the frequency matrix of the Mori equation for this set of variables and transforming yields the equation

$$
\begin{aligned}
\frac{d B(t)}{d t}=\left(\begin{array}{ccc}
i \Omega_{1} & 0 & 0 \\
0 & -i \Omega_{1} & 0 \\
0 & 0 & 0
\end{array}\right) B(t)-\int_{0}^{t} \Phi_{1}(s) B(t-s) d s \\
+F(t) .
\end{aligned}
$$

Here $B(t)$ is a column vector with components $\left(B_{1}(t), B_{2}(t), B_{3}(t)\right)$ where 


$$
\begin{aligned}
& \mathrm{B}_{1}=\dot{\mathrm{S}}(\mathrm{k})+i \frac{\Omega_{\mathrm{o}}^{2}}{\Omega_{1}} \mathrm{~S}(\mathrm{k})-\frac{1}{\Omega_{1}} \mathrm{G}(\mathrm{k}), \\
& \mathrm{B}_{2}=\dot{\mathrm{S}}(\mathrm{k})-1 \frac{\Omega_{\mathrm{o}}^{2}}{\Omega_{1}} \mathrm{~S}(\mathrm{k})+\frac{i}{\Omega_{1}} \mathrm{G}(\mathrm{k}), \\
& \mathrm{B}_{3}=\delta_{2} \mathrm{~S}(\mathrm{k})+\mathrm{G}(\mathrm{k}) .
\end{aligned}
$$

The mode frequency is given by

$$
\Omega_{1}^{2}=\Omega_{0}^{2}+\delta_{2}=\delta_{1}+\delta_{2}
$$

The random force $F(t)$ is given by

$$
F(t)=\left(\begin{array}{c}
-\frac{1}{\Omega_{1}} \\
\frac{i}{\Omega_{1}} \\
1
\end{array}\right) f_{1}(t)
$$

where

$$
f_{1}=s^{(3)}(k)+\frac{\left\langle\omega^{4}\right\rangle}{\left\langle\omega^{2}\right\rangle} \dot{s}(k) .
$$

The memory function matrix is

$$
\begin{aligned}
& \Phi_{1}(t)=\frac{1}{2 \Omega_{1}^{3}}\left(\begin{array}{ccc}
\delta_{2} \Omega_{1} & -\delta_{2} \Omega_{1} & -21 \Omega_{0}^{2} \\
-\delta_{2} \Omega_{1} & \delta_{2} \Omega_{1} & 2 i \Omega_{0}^{2} \\
i \delta_{2} \Omega_{1}^{2} & -i \delta_{2} \Omega_{1}^{2} & 2 \Omega_{0}^{2} \Omega_{1}
\end{array}\right) \Phi_{1}(t) \\
& \phi_{1}(t)=\frac{\left(f_{1}(t), f_{1}^{*}\right)}{\left(G(k), G(k)^{*}\right)}=\delta_{3} \frac{\left(f_{1}(t), f_{1}^{*}\right)}{\left(f_{1}, f_{1}^{*}\right)}=\delta_{3} \psi_{1}(t) .
\end{aligned}
$$

The interesting feature of $(7.26)$ is the appearance of a zero diagonal element in the frequency matrix, or in other words, the appearance 
of a diffusive mode, which was not observed in the two-variable theory of the previous section. Before considering time scales in the threevariable theory, we examine the relaxation function $\psi_{1}(t)$. From Chapter VI (or Mori 1965b) the Laplace transform $\psi_{1}(z)$ and the Laplace transform $\psi(z)$ of the random force relaxation function $\psi(t)$ of the two-variable theory are known to be related by the exact equation

$$
\psi(z)=\frac{1}{z+\delta_{3} \psi_{1}(z)}
$$

We may therefore obtain $\psi_{1}(z)$ simply by using the Gaussian approximation for $\psi(t)$ as defined by (7.19) and the assumptions stated there. Note that the breakdown of the two-variable theory for $k \ll k_{c}$ does not affect the validity of the Gaussian approximation for $\psi(t)$, since (7.14) and (7.19) are based on the behavior of the exact $\delta_{3}$ for the classical chain. The relaxation time $\tau_{2}$ of the random force $f_{1}(t)$ is estimated as in (7.17), and using (7.32) we find

$$
\tau_{2}=\psi_{1}(z=0)=\left(\frac{2}{\pi \delta_{3}}\right)^{\frac{1}{2}}
$$

Notice that $\tau_{2}$, like $\tau_{1}$, depends on $\delta_{3}$ and may therefore be considered insensitive to changes in wavevector $k$.

We now return to $(7.26)$ and consider the criteria for the decoupling of the convolution integral in the same way as we examined (7.13). The criteria (7.15) and (7.16) now apply to the normalized relaxation function $\left(B(t), B^{*}\right)\left(B, B^{*}\right)^{-1}=R_{1}(t)$ of the variables $B(t)$. Suppose that the long-time behavior of $R_{1}(t)$ is dominated by the quantity $\Gamma_{1}$ in the matrix of damping constants. Then the time scale separation criterion (7.16) becomes in the present case 


$$
\frac{1}{\Gamma_{1}} \gg \tau_{2}
$$

Let us assume that (7.34) is valid. Then we may decouple the convolution integral in (7.26) exactly as specified in (7.18). An important difference between the result $(7.21)$ of decoupling in the two-variable theory and decoupling in the three-variable theory arises because of the zero element in the frequency matrix $1 \omega_{1}$ in the three-variable equation (7.26). To see this difference clearly we rewrite the memory function $\Phi_{1}(t)$ of $(7.31)$ as

$$
\Phi_{1}(t)=M \phi_{1}(t),
$$

where $M$ is the constant matrix of coefficients $\ln (7.31)$. Now we carry out the decoupling and find

$$
\Lambda=\int_{0}^{\infty} \Phi_{1}(t) e^{-i \omega_{1} t} d t=M\left(\begin{array}{cccc}
\phi_{1}\left(z=1 \Omega_{1}\right) & 0 & 0 \\
0 & \phi_{1}\left(z=-i \Omega_{1}\right) & 0 \\
0 & 0 & \phi_{1}(z=0)
\end{array}\right),
$$

where $\phi_{1}(z)=\int_{0}^{\infty} e^{-z t} \phi_{1}(t) d t$, is the Laplace transform of $\phi_{1}(t)$, and the frequency matrix $i w_{1}$ is the matrix coefficient of $B(t)$ on the righthand side of $(7.26)$.

The equation of motion (7.26) may now be rewritten as

$$
\frac{d B(t)}{d t}=\left(i \omega_{1}-\Lambda\right) B(t)+F(t)
$$

We now must diagonalize the matrix $\left(i \omega_{1}-\Lambda\right)$ to obtain the collective mode frequencies and damping. However the results of such a dlagonalization are a set of cumbersome algebraic expressions which are not very illuminating. We therefore follow the procedure of (7.24) and neglect the imaginary part of $\Lambda$. In the limiting regions of high and low $k$ the 
latter approximation gives essentially the same results as the exact diagonalization. We define the quantities

$$
\begin{aligned}
& \mu_{1}=\operatorname{Re} \phi_{1}\left(z=i \Omega_{1}\right), \\
& \mu_{2}=\phi_{1}(z=0), \\
& \gamma_{1}=\frac{\delta_{2} \mu_{1}}{2 \Omega_{1}^{2}}, \\
& \gamma_{2}=\frac{\delta_{1} \mu_{2}}{2 \Omega_{1}^{2}} .
\end{aligned}
$$

Diagonalizing the matrix under the above approximation we obtain the collective mode equation of motion

$$
\frac{\mathrm{dC}(t)}{\mathrm{dt}}=\left(\begin{array}{ccc}
i \Omega_{2}-\gamma_{1} & 0 & 0 \\
0 & -i \Omega_{2}-\gamma_{1} & 0 \\
0 & 0 & -\gamma_{2}
\end{array}\right) \quad c(t)+F_{1}(t)
$$

where the mode variables are the components $\left(c_{1}, c_{2}, c_{3}\right)$ of $c$, given by

$$
\begin{aligned}
C_{1}(t)= & (1+q) \dot{S}(k, t)+\frac{i \Omega_{0}^{2}}{\Omega_{1}}(1-q) S(k, t)-\frac{i}{\Omega_{1}} G(k, t), \\
C_{2}(t)= & (1-q) \dot{S}(k, t)-\frac{1 \Omega_{1}^{2}}{\Omega_{1}}(1+q) S(k, t)+\frac{i}{\Omega_{1}} G(k, t), \\
C_{3}(t)= & \delta_{2} S(k, t)+G(k, t), \\
q= & \frac{i \gamma_{1}}{\Omega_{1}+\Omega_{2}},
\end{aligned}
$$

and the mode frequency is given by

$$
\Omega_{2}^{2}=\Omega_{1}^{2}-\gamma_{1}^{2}
$$


The damping constants $\gamma_{1}, \gamma_{2}$ may be obtained from (7.32), (7.19) and $(7.38)$ as

$$
\begin{aligned}
& \gamma_{1}=\left(\frac{8 \delta_{3}}{\pi}\right)^{\frac{1}{2}} \frac{\delta_{2}}{\left(\delta_{1}+\delta_{2}\right)} e^{\delta_{1} / 2 \delta_{3}}, \\
& \gamma_{2}=\left(\frac{2 \delta_{3}}{\pi}\right)^{\frac{1}{2}} \frac{\delta_{1}}{\left(\delta_{1}+\delta_{2}\right)} .
\end{aligned}
$$

Using (7.42) we now examine the regions $k<<k_{c}$ and $k>>k_{c}$ at low temperatures. The values of the $\delta^{\prime}$ 's are estimated to be equal to the exact values for the classical chain.

The region $k \ll k_{c}$ is of central interest here. We see that $\gamma_{1}$ increases as $k$ decreases (in much the same way as $\gamma$ in (7.22)), but that $\gamma_{2}$ is proportional to $\frac{\delta_{1}}{\delta_{2}}$ for $k \ll k_{c}$. Hence for very low $k, \gamma_{2}$ becomes small and in the low $k$ region the time decay of the relaxation matrix of $C(t)$ is dominated by the slow time scale $\frac{1}{\gamma_{2}}$. Thus even for low $k \ll k_{c}$ the criterion $\frac{1}{\Gamma_{1}} \gg \tau_{2}$ is satisfied with $\Gamma_{1}=\gamma_{2}$, and the separation of time-scales remains valid.

In the region $k>k_{c}$ the quantity $\gamma_{2} \sim \delta_{3}^{\frac{1}{2}}$, and so the mode variable $C_{3}(t)$ (which is essentially equal to $G(k, t)$ for $k>k_{c}$ ) has a decay time $\frac{1}{\gamma_{2}} \sim \delta_{3}^{-\frac{1}{2}}$ of the same order of magnitude as $\tau_{2}$. However the modes $C_{1}(t), C_{2}(t)$ have a decay time for $k \gg k_{c}$ which is governed by $\gamma_{1} \sim \frac{\delta_{2}}{\delta_{1}} \delta_{3}^{\frac{1}{2}}$, and since $\delta_{2} \ll \delta_{1}$ (for $k \gg k_{c}$ ) we see that $\frac{1}{\gamma_{1}} \gg \tau_{2}$. In the region $k \gg k_{c}$ we now have that the relaxation matrix of $c(t)$ decays on the time scale given by $\Gamma_{1}=\gamma_{1}$.

The above arguments show that the separation of time scales assumed in (7.34) provides a consistent description of collective modes 
in the linear Heisenberg chain for the whole range of wavevectors $k$. For $k \ll k_{c}$ a diffusive mode dominates the relaxation spectrum, while for $k \gg k_{c}$ the dominant modes are damped oscillatory motions. The high $k$ regime can also be described by the two-variable theory of the preceding section with results similar to the three-variable theory for $k>k_{c} \cdot$

\section{DISCUSSION}

We have shown that the variables $s(k, t), \dot{s}(k, t)$ and $\ddot{s}(k, t)$ provide a consistent description of collective dynamics in the Heisenberg chain within the Mori formalism. For values of wavevector $k$ higher than $k_{c}$ the variables $S(k, t)$ and $\dot{S}(k, t)$ couple in a manner analogous to the coupling of coordinate and momentum (or velocity) in the stochastic simple harmonic oscillator. The high $k\left(>k_{c}\right)$ modes are damped oscillatory motions of $s(k, t)$ and $\dot{s}(k, t)$ with a well-defined mode frequency and damping. As the wavevector $k$ decreases to values $k \ll k_{c}$ the motion of the coupled mode variables $C_{1}(t), C_{2}(t)$ becomes very rapidly damped with a short time scale. On the other hand the variable $G(k, t)$, which is the part of $\ddot{s}(k, t)$ orthogonal to $s(k, t)$ and $\dot{s}(k, t)$, has almost the opposite behavior. For $k>k_{c}$ the motion of $G(k, t)$ is very highly damped, but for $k \ll k_{c}$ the motion of $G(k, t)$ is on a much slower time scale than that of $C_{1}(t)$ and $C_{2}(t)$. In the low $k\left(<k_{c}\right)$ regime the dominant modes consist of the damped motion of $G(k, t)$.

Our results for the mode frequencies and damping are simtlar to the results obtained by Tomita and Mashiyama (1972) and Lovesey (1974). In particular the results of our two-variable theory are very much the 
same as Tomita and Mashiyama's result for $k \gg k_{c}$, while their results for $k \ll k_{c}$ are essentially the same as the result of our three-variable theory for the small k regime. The functions $\psi(t), \psi_{1}(t)$ which arise in our theory are precisely the same as the functions which arise in the second and third stages of a continued fraction approximation. For $k \gg k_{c}$ the two-variable theory presented here is formally identical with Tomita and Mashiyama's two-stage continued fraction. For low $k \ll k_{c}$ however, they use an explicitly frequency dependent termination function while we extend the set of varlables. Since the results of both procedures appear to be consistent we conclude that the use of an explfcitly frequency dependent termination function results in an extension of the range of frequencles over which the termination approximation is valid. Lovesey (1974) uses a three stage continued fraction expansion, which is formally equivalent to our use of derivatives of $S(k, t)$ to second order. Lovesey uses a short-time expansion of the spin relaxation function $R_{0}(k, t)$ in order to approximate $\psi_{1}(z=0)$. Our objective in doing the two- and three-variable was to describe the dynamics of the good collective modes, which are not explicitly described by approximation methods for the lineshape. The dynamical equations of motion are intended to motivate a physical description of collective modes in the linear Heisenberg chain. It seems 1ikely that a check of our time-scale separation criteria would be a useful test of our description of the collective modes. Such a test could be stratghtforwardly done for the classical Heisenberg chain by numerically computing the necessary correlation functions, in the manner outlined by Steiner et. al. (1976). 
REFERENCES

Abrams, P. 1976. Theor. Pop. Biol. 8, 356.

Aiken, R. C. and Lapidus, L. 1974. Int. J. Systems Sc1. 4, 691.

Akcasu, A. Z. and Duderstadt, J. J. 1969. Phys. Rev. 188, 479.

Ayala, F. 1969. In Essays in evolution and genetics in honor of Theodosius Dobzhansky. M. K. Hecht and W. C. Steere (eds.). Appleton-Century-Crofts, New York.

Bailey, N. T. J. 1962. The elements of stochastic processes, John Wiley, New York.

Bartlett, M. S. 1973. In The mathematical theory of the dynamics of biological populations. M. S. Bartlett and R. W. Hiorns (eds.). Academic, London.

Berne, B. J. and Harp, G. D. 1970. Adv. Chem. Phys. 16, 63.

Botkin, D. B. and Sobe1, M. J. 1974. Am. Nat. 109, 625 .

Brauer, F. and Sanchez, D. A. 1975. Theor. Pop. Biol. $\underline{8}, 12$.

Cohen, J. K. and Lew1s, R. M. 1967. J. Inst. Math. App1. 3, 266.

Crow, J. F. and Kimura, M. 1970. An introduction to population genetics theory. Harper and Row, New York.

Culver, D. C. 1975. In Ecosystem analysis and prediction. S. A. Levin (ed.). SIAM, Philadelphia.

DeBaggis, H. F. 1955. Ann. of Math. Studies, r.z. 29, Princeton Univ. Press, Princeton.

noob, J. L. 1953. Stochastic processes. Wiley, New York.

E1ton, C. S. 1958. The ecology of invasions by animals and plants. Methuen, London.

Feldman, M. W. and Roughgarden, J. 1975. Theor. Pop. Biol. Z, 197.

Feller, W. 1952. Ann. Math. 55, 468.

Field, R. J. and Noyes, R. M. 1974. J. Chem Phys. 60, 1877. 
F1sher, M. E. 1964. Am. J. Phys. 32, 343.

Gantmacher, F. R. 1960. The theory of matrices. Chelsea, New York.

Gause, G. F. 1934. The struggle for existence. Williams and Wilkins, Baltimore.

Gause, G. F. and Witt, A. A. 1935. Am. Nat. 69, 596.

Gilpin, M. E. 1974. J. Theor. Biol. 44, 35.

Goel, N. S., Maitra, S. C., and Montro11, E. W. 1971. Rev. Mod. Phys. 43, 231 .

Goodwin, B. C. 1970. J. Theor. Biol. 28, 375.

Griego, R. and Hersh, R. 1971. Trans. Amer. Math. Soc. 156, 405.

Hahn, W. 1967. Stability of motion. Springer-Verlag, New York.

Haken, H. 1975. Rev. Mod. Phys. 47, 67.

Haken, H. 1976. Z. Physik. B24, 321

Harper, J. L. 1967. J. App1. Eco1. 4, 267.

Harper, J. L. 1969. The role of predation in vegetational diversity, Brookhaven Symposium in Blology No. 22. N.B.S., Springfield, Va.

Holling, C. S. 1973. Ann. Rev. Eco1. Syst. $\underline{4}, 1$.

Hone, D. and Richards, P. M. 1974. Ann. Rev. Mater. Sci. 4, 337.

Hughes, R. D. and Gilbert, N. 1970. J. Anim. Ecol. 37, 553.

Hutchinson, G. E.1953. Am. Nat. 93, 145 .

Inselberg, A. and Dula, G. 197 . Amer. Math. Mthly. 81, 1102.

Jeffreys, C. 1975. Theor. Pop. Biol. $\underline{7}, 149$.

Karlin, S. 1974. A first course in stochastic processes. Academic, New York.

Kawasaki, K. 1975. In Phase transitions and critical phenomena, Vo1. 5A, C. Domb and M. S. Green (eds.), Academic, London.

Kerner, E. H. 1964. Math. Biophys. 23, 141.

Keyes, T. and Oppenheim, I. 1973. Phys. Rev. A8, 937. 
Kim, K. and Nelkin, M. 1971. Phys. Rev. A4, 2065.

Kimura, M. 1955. Stochastic processes and distribution of gene frequencies under natural selection, Cold Spring Harbor Symp. Quant. Biol., 20 .

Kivelson, D. and Ogan, K. 1973. Adv. in Magnetic Resonance, $\underline{7}, 72$.

Koch, A. L. 1974a. J. Theor. Biol. 44, 387.

Koch, A. I. 1974b. J. Theor. Biol. 44, 373.

Krylov, N. and Bogoliubov, N. N. 1943. Ann. of Math. Studies, no. 11, Princeton Univ. Press, Princeton.

Kubo, R. 1963. J. Math. Phys. 4, 174.

Kubo, R., Matsuo, K., and Kitahara, K. 1973. J. Stat. Phys. 9, 51.

Lax, M. 1966. Rev. Mod. Phys. 38, 561.

Leigh, F. 1968. In Some mathematical problems in biology. The American Mathematical Society, Providence.

Leigh, E. 1971. Adaptation and diversity. Freeman, Cooper, San Francisco.

Leslie, P. H. 1948. Biometrica. 35, 213.

Levin, S. A. and Paine, R. T. 1974. Proc. Nat. Acad. Sci. 71, 2744.

Levins, R. 1966. Amer. Sci. 54, 421.

Levins, R. 1969. Proc. Nat. Acad. Sc1. 62, 1061.

Levins, R. 1974. Ann. N. Y. Acad. Sci. 231, 123.

Levins, R. 1975. In Ecosystem analysis and prediction, S. A. Levin (ed.), SIAM, Philadelphia.

Lewontin, R. C. 1969. The meaning of stability, Brookhaven Symposium in Biology No. 22, N.B.S., Springfield, Va.

Lewontin, R. C. 1972. Nature. 236, 181.

Lewontin, R. C. 1974. The genetic basis of evolutionary change. Columbia Univ. Press, New York.

Lewontin, R. C. and Cohen, D. 1969. Proc. Nat. Acad. Sci. 62, 1056.

Lotka, A. J. 1954. Elements of physical biology. Dover, New York. 
Lovesey, S. W. 1974. J. Phys. C. ㄱ, 2008.

Lovesey, S. W. and Meserve, R. A. 1972. Phys. Rev. Lett. 28, 614.

Ludwig, D. 1975. SIAM Review. 17, 605.

Ludwig, D. 1976. Math. Biosci. 23, 47.

MacArthur, R. H. 1955. Ecology. 36, 533.

MacArthur, R. H. 1969. Proc. Nat. Acad. Sci. 64, 1369.

MacArthur, R. H. 1970. Theor. Pop. Biol. $1,1$.

MacArthur, R. H. 1972. Geographical ecology. Harper \& Row, New York.

Margalef, R. 1958. Gen. Syst. $\underline{3}, 36$.

May, R. M. 1974. Stability and complexity in model ecosystems. 2nd ed. Princeton Univ. Press, Princeton.

May, R. M. 1975. In Ecological stability, M. B. Usher and M. H. Williamson (eds.), Chapman and Ha11, London.

Miller, G. F. 1962. Proc. Camb. Phil. Soc. 58, 588.

Minorsky, N. 1962. Nonlinear oscillations. Van Nostrand, Princeton.

Mori, H. 1965a. Prog. Theor. Phys. 33, 423.

Mori, H. 1965b. Prog. Theor. Phys. 34, 399.

Mori, H. 1973. Prog. Theor. Phys. 49, 764.

Mori, H. 1974. Prog. Theor. Phys. 52, 433.

Mortensen, R. E. 1969. J. Stat. Phys. 1, 271.

Mountford, M. D. 1973. In The mathematical theory of the dynamics of blological populations, M. S. Bartlett and R. W. Hiorns (eds.). Academic, London.

Namkoong, G. 1974. Theor. Pop. Biol. $\underline{3}, 507$.

Nordholm, S. J. K. and Zwanzig, R. 1975. J. Stat. Phys. 13, 347.

Papanicolau, G. C. 1972. SIAM J. Appl. Math. 21, 13.

Papanicolau, G. C. 1973. Amer. Math. Mthly. 80, 526.

Papanicolau, G. C. and Kohler, W. 1974. Comm. Pure and Appl. Math. 26. 641 . 
Parker, R. A. 1973. In The mathematical theory of the dynamics of biological populations, M. S. Bartlett and R. W. Hiorns (eds.). Academic, London.

Parker, R. A. 1975. Int. J. Systems Sci. $\underline{6}, 197$.

Patten, B. C. 1976. Am. Nat. 109, 529.

Penrose, 0. 1970. Foundations of statistical mechanics. Pergamon, Oxford.

Pianka, E. R. 1974. Evolutionary ecology- Harper \& Row, New York.

Pielou, E. C. 1969. An introduction to mathematical ecology. Wiley-Interscience, New York.

Pielou, E. C. 1975. Ecological diversity. John Wiley, New York.

Pimente1, D. 1968. Science 159, 1432.

Poole, R. T. 1974. An introduction to quantitative ecology. McGraw Hill, New York.

Preston, F. W. 1969. Diversity and stability in the blological world, Brookhaven Symposium in Biology No. 22. N.B.S., Springfield, Va.

Quirk, J. P. and Ruppert, R. 1965. Rev. Econ. Studies. 32, 311.

Reddy, V. T. N. 1974. J. Stat. Phys. 13,61 .

Ricklefs, R. 1973. Ecology. Chiron, Newton, Mass.

Roughgarden, J. 1975. Am. Nat. 109, 713.

Roughgarden, J. 1976. Theor. Pop. B1o1. $\underline{9}, 388$.

Schnelder, W. R. 1976. Z. Physik. B24, 135.

Seifert, R. P. and Seifer, F. H. 1976. Am. Nat. 110, 461.

Siljak, D. D. 1975. I. E. E. E. Trans. SMC-3, 415.

Siljak, D. D. 1976. Math. Biosc. 25, 25.

Simon, H. A. 1973. In Heirarchy theory, H. H. Pattee (ed.). Braziller, New York.

Slobodkin, L. B. 1961. The growth and regulation of antmal numbers. Holt, Rinehart and Winston, New York.

Slobodkin, I. B. 1969. Quart. Rev. Blo1. 49, 181. 
Steiner, M., Villain, J. and Windsor, C. G. 1976. Adv. In Physics. $\underline{25}, 87$.

Stewart, F. M. and Levin, B. R. 1973. Am. Nat. 107, 171.

Stratonovitch, R. L. 1966. Conditional Markov processes and their application to the theory of optimal control. American E1sevier, New York.

Strobeck, C. 1973. Ecology. 54, 650.

Takagahara, T. 1976. Prog. Theor. Phys. 55, 48.

Takeyama, K. 1975. Prog. Theor. Phys. 54, 700.

Tomita, H. and Mashiyama, H. 1972. Prog. Theor. Phys. 48, 1133.

Tomita, K. and Mashiyama, H. 1974. Prog. Theor. Phys. 51, 1312.

Tuljapurkar, S. D. and Semura, J. S. 1975. Nature. 257, 388.

Van Kampen, N. G. 1965. In Fluctuation phenomena in solids, R. F. Burgess (ed.). Academic, New York.

Vent'sel, A. D. and Friedlin, M. I. 1970. Russian Math. Surveys. $\underline{25}, 1$.

Volterra, V. 1926. Mem. Acad. Lincei. 2, 31.

Walter, C. W. 1973. Bull. Math. Biol. 36, 215.

Weiss, G. H. and Dishon, M. 1975. J. Stat. Phys. 13, 145.

Wilson, K. G. 1975. Phys. Reports. 12C, 75.

Wong, E. and Zakai, M. 1969. Internat. J. Engrg. Sci. 3, 213.

Wright, S. 1973. Evolution and the genetics of populations. Univ. of Chicago, Chicago.

Wynne-Edwards, V. C. 1952. Auk. 69, 352. 
APPENDIX A

In the first part of this appendix we summarize some theorems on stability which are used extensively in Chapters III to $V$. In the second part we deal with an extension of the results of Chapter IV to many-species interactions.

Consider a nonlinear deterministic set of equations for the tIme development of the vector $\vec{N}=\left(N_{1}, \ldots, N_{m}\right)$,

$$
\frac{d \vec{N}}{d t}=\vec{g}(\vec{N})
$$

Suppose (A1) has an equilibrium point $\vec{N}=\vec{N}^{*}$ where $\vec{g}\left(\vec{N}^{*}\right)=t$. We rewrite (AI) in terms of the deviations from equilibrium $\vec{x}=\vec{N}-\vec{N}^{*}$,

$$
\begin{aligned}
\frac{d \vec{x}}{d t} & =\vec{g}\left(\vec{N}^{*}+\vec{x}\right), \\
& =\overrightarrow{A x}+\text { (nonlinear terms), }
\end{aligned}
$$

where $A=(\partial \vec{g} / \partial \vec{N})$ evaluated at $\vec{N}=\vec{N}^{*}$. The system (A2) has an equilibrium at $\vec{x}^{*}=\overrightarrow{0}$, the origin. Any solution of (A2) which starts at $\vec{x}_{0}$ at $t=t_{0}$ may be written $\vec{x}\left(t, \vec{x}_{0}, t_{0}\right)$.

The origin of (Al) is stable if for any $\varepsilon>0$ there exists a $\delta>0$ such that if $\left|\vec{x}_{0}\right|<\delta$ then $\left|\vec{x}\left(t, \vec{x}_{0}, t_{0}\right)\right|<\varepsilon$, for all $t \geq t_{0}$. The origin of (A1) is asymptotically stable if it is stable and if there exists a $\delta_{1}>0$ such that $\left|\vec{x}_{0}\right|<\delta_{1}$ implies $\lim _{t \rightarrow \infty}\left|\vec{x}\left(t, \vec{x}_{0}, t_{0}\right)\right|=0$. If we neglect the nonlinear terms in (A2), the 11near equation is stable (asymptotically stable) about the origin if and only if the real parts of the eigenvalues of $A$ are nonpositive (negative). An alternative condition for asymptotic stability is that there exist a symetric 
positive-definite matrix $P$ such that

$$
A^{\prime} P+P A=-Q
$$

for any given symmetric positive definite Q.

For the full nonlinear system only sufficient conditions exist. The origin is stable (asymptotically stable) for (Al) if there exists a positive definite function $V(\vec{x})$ with $V(\overrightarrow{0})=0$ such that $d V / d t$ is negative semi-definite (negative definite). Such a function $v(\vec{x})$ if it exists is called a Liapunov function.

We now extend the theory of Chapter IV to cover many-species interactions. We write an n-species Lotka-Volterra model as

$$
\frac{\mathrm{dN}_{i}}{\mathrm{dt}}=\frac{\mathrm{r}_{i} \mathrm{~N}_{i}}{\mathrm{~K}_{i}}\left[\mathrm{~K}_{i}-\sum_{j} a_{i j} \mathrm{~N}_{j}\right], i, j=1,2, \ldots, \mathrm{n}
$$

We change variables to $z_{i}=1 \mathrm{nN}_{1}$, and consider two trajectories: one is specified by the inftial point $\vec{z}(0)$ and at later times as $\vec{z}(t)$, while the other is specified by initial point $\vec{z}(0)+\vec{x}(0)$ and at later times as $\vec{z}(t)+\vec{x}(t)$. Then, as in (4.3), we find that the $x_{i}(t)$ obey the equations

$$
\frac{d x_{i}}{d t}=-\frac{r_{i}}{k_{i}}\left\{\sum_{j} a_{i j} N_{i}\left[\exp \left(x_{j}\right)-1\right]\right\}
$$

The discussion following (4.3) still holds: we wish to examine the behavior of $|\vec{x}(t)|$ as $t \rightarrow \infty$, in particular whether $|\vec{x}(t)| \rightarrow 0$ as $t \rightarrow \infty$. The point $\vec{x}^{*}=(\overrightarrow{0})$ is an equilibrium point for (A5). The Liapunov function (4.5) serves here as well,

$$
V(\vec{x})=\sum_{i}\left[\exp \left(x_{i}\right)-x_{i}-1\right],
$$

with 


$$
\frac{d V}{d t}=\sum_{i, j}\left[\exp \left(x_{i}\right)-1\right] b_{i f}\left[\exp \left(x_{j}\right)-1\right]
$$

and

$$
b_{i j}=-a_{i j} N_{i} \frac{r_{i}}{K_{i}}
$$

The necessary and sufficient condition for $V(\vec{x})$ to ensure stability about $\vec{x}^{*}$ is that $\left(B+B^{\prime}\right)$ be negative-defintte, where $B$ is the matrix $\left(b_{i j}\right)$ and $B^{\prime}$ is the transpose of $B$. Given this condition trajectories of (A5) will converge in time. The rest of our discussion from Chapter IV may now be extended to the model (A4). 
APPENDIX B

Here we set out the two rigorous descriptions of the 'white noise process' and the diffusion equation (5.8).

The Ito equation represents white noise in terms of a k-dimensiona1 Wiener (Brownian motion) process $\vec{B}(t)$ whose components $B_{j}(t), j=1$, $\ldots, k$, have the properties

$$
\begin{aligned}
E\left[\Delta B_{j}(t)\right] & =E\left[B_{j}(t+\Delta t)-B_{j}(t)\right]=0, \\
E\left[\Delta B_{i}(t) \Delta B_{j}(t)\right] & =d_{1 j} \Delta t, \quad 1, j=1, \ldots, k,
\end{aligned}
$$

where $d_{i j}$ have the same properties as in equation (5.7).

The white noise process $\vec{Z}(t)$ is heuristically interpreted as the time derivative of the Wiener process $\vec{B}(t)$. However, the Wiener process is almost never differentiable, so the Ito form of the stochastic equation (5.7) is written in terms of differentlals as

$$
d \vec{X}=\vec{G}(\vec{X}) d t+\sqrt{\varepsilon} F(\vec{X}) d \vec{B}(t)
$$

The probability density $f(\vec{x}, t)$ for the Ito process $\vec{x}(t)$ in (B2) obeys the Ito diffusion equation which has the form $(5.8)$ with the drift and diffusion coeficients defined as

$$
\begin{aligned}
b_{i}(\vec{x}) & =\lim _{\Delta t \rightarrow 0} \frac{1}{\Delta t} E\left[x_{i}(t+\Delta t)-x_{i}(t) \mid \vec{x}(t)=\vec{x}\right] . \\
& =G_{i}(\vec{x}) \\
\varepsilon a_{i j}(\vec{x}) & =\lim _{\Delta t \rightarrow 0} \frac{1}{\Delta t} E\left[\left(x_{i}(t+\Delta t)-x_{i}(t)\right)\left(x_{j}(t+\Delta t)-x_{j}(t) \mid \vec{x}(t)=\vec{x}\right]\right. \\
& =\varepsilon \sum_{p, q} d_{p q} F_{i p} F_{j q}, \quad 1, j=1, \ldots, m ; p, q=1, \ldots, k .
\end{aligned}
$$

The Stratonovitch equation has been extensively discussed in terms of a definition of stochastic integrals which differs from Ito's 
definition (Mortensen 1969). However we will follow Papanicolau and Kohler (1974) who have obtained vigorously a diffusion equation of the Stratonovitch form, and have improved on the results of Wong and Zakai (1969). Papanicolau and Kohler use a set of $\mathrm{k}$ stationary zero-mean processes $Y_{j}(t), j=1, \ldots, k$. The process $\vec{Y}(t)=\left\{Y_{j}(t)\right\}$ is a mixing process, which means essentially that it has a finite memory. Define the matrix

$$
\gamma_{i j}=\int_{0}^{\infty} E\left[Y_{i}(s) Y_{j}(0)\right] d s
$$

Now introduce a parameter $\beta$, and consider the processes $Y_{j}^{(\beta)}(t)=$ $\frac{1}{\beta} Y_{j}\left(\frac{t}{\beta^{2}}\right)$ in the limit as $\beta \rightarrow 0$. These processes converge to the Gaussian 'white noise process' $\vec{Z}(t)$. The stochastic differential equation (5.7) is written in the Papanicolau-Kohler form as

$$
\frac{\mathrm{d}}{\mathrm{dt}}^{(\beta)}=\vec{G}\left(\overrightarrow{\mathrm{X}}^{(\beta)}\right)+\sqrt{\varepsilon} \mathrm{F}\left(\overrightarrow{\mathrm{X}}^{(\beta)}\right) \frac{1}{\beta} \overrightarrow{\mathrm{Y}}\left(\frac{\mathrm{t}}{\beta^{2}}\right) .
$$

In the 1imit $\beta \rightarrow 0$ the solution process $\vec{x}^{(\beta)}$ of (B5) converges to a diffusion Markov process whose drift and diffusion coefficients are given by

$$
\begin{aligned}
& b_{i}(\vec{x})=G_{i}(\vec{x})+\varepsilon \sum_{p, q, j} \gamma_{p q} F_{j p} \frac{\partial F}{\frac{i q}{\partial x_{j}}} \\
& a_{i j}(\vec{x})=\sum_{p, q} \gamma_{p q} F_{i p} F_{j q}, \quad i, j=1, \ldots, n ; p, q=1, \ldots, k .
\end{aligned}
$$

The coefficients (B6) when inserted into the diffusion equation (5.8) yield the Stratonovitch-Papanicolau-Kohler diffuston equation. 
APPENDIX C

We present here details of the solution procedure discussed in Chapter $V$, and of the formula for the minimum eigenvalue $\lambda_{0}$. The material in this appendix essentially reproduces some of the analysis in DL 75 and is included mainly for completeness.

A preliminary point concerns the two forms of the diffuston equation given in Appendix B. Consider the drift coefficient for the Stratonovitch equation glven in (B6). If we define the functions

$$
\begin{aligned}
c_{i}(\vec{x}) & =\sum_{p, q, j} \gamma_{p q} F_{j p} \frac{\partial F_{i q}}{\partial x_{j}} \\
i, j & =1, \ldots, m ; p, q=1, \ldots, k,
\end{aligned}
$$

then the Stratonovitch drift coefficient in (B6) reduces to the Ito drift coefficient in (B3) if we set $C_{i}(\vec{x})=0$, all i. For this reason we use the Stratonovitch operator $\mathrm{L}_{S}$ in the ensuing discussion and note that al1 results translate to the Ito case if the $C_{1}(\vec{x})$ are set equal to zero.

We begin by inserting the ansatz (5.9), $g=h \exp (-v / \varepsilon)$, Into the time-independent equation $L_{s} g(\vec{x})=0$, and obtain 


$$
\begin{aligned}
& L_{s} g=\frac{1}{\varepsilon} \exp \left(\frac{-v}{\varepsilon}\right) h\left\{\frac{1}{2} \sum_{i, j} a_{i j} \frac{\partial v}{\partial x_{1}} \frac{\partial v}{\partial x_{i}}+\sum_{1} G_{i} \frac{\partial v}{\partial x_{i}}\right\} \\
& -\varepsilon^{0} \exp \left(\frac{-v}{\varepsilon}\right)\left\{\sum_{i, j}\left(a_{i j} \frac{\partial v}{\partial x_{j}}+G_{i}\right) \frac{\partial h}{\partial x_{i}}+\sum_{i, j}\left(\frac{\partial a_{1 j}}{\partial x_{i}} \frac{\partial v}{\partial x_{j}}\right.\right. \\
& \left.\left.+\frac{1}{2} a_{i j} \frac{\partial^{2} v}{\partial x_{i} \partial x_{j}}+\frac{\partial G_{i}}{\partial x_{i}}-c_{i} \frac{\partial v}{\partial x_{i}}\right) h\right\} \\
& +O\left(\varepsilon \exp \left[\frac{-v}{\varepsilon}\right]\right)=0 \text {, } \\
& i, j=1, \ldots, m \text {. }
\end{aligned}
$$

If the term in (C2) proportional to $\frac{1}{\varepsilon}$ is set equal to zero we get equation (5.10) for $v(\vec{x})$, while setting the term in $\varepsilon^{0}$ to zero gives an equation for $h(\vec{x})$. Before considering $h(\vec{x})$ we discuss (5.10) a little further.

Define the quantities $p_{i}=\frac{\partial v}{\partial x_{i}}$, all $i$, and then rewrite $(5.10)$ as

$$
E(\vec{x}, \vec{p}, v)=\frac{1}{2} \sum_{i, j} a_{i j} p_{i} p_{j}+\sum_{i} r_{i} p_{i}=0,1, j=1, \ldots, m .
$$

The characteristic differential equations corresponding to the partial differential equation (C3) may be written down in terms of an independent variable $\sigma$ as (Courant \& Hilbert 1960)

$$
\begin{aligned}
& \frac{d x_{i}}{d \sigma}=\frac{\partial E}{\partial p_{i}}=\sum a_{i j} p_{j}+G_{i}, \\
& \frac{d p_{i}}{d \sigma}=-\frac{\partial E}{\partial x_{i}}=-\sum_{j, \ell} \frac{\partial a_{j \ell}}{\partial x_{i}} p_{j} p_{\ell}-\sum_{j} \frac{\partial G_{j}}{\partial x_{i}} p_{j}, \\
& \frac{d v}{d \sigma}=\sum_{i} p_{i} \frac{\partial E}{\partial p_{i}}=\frac{1}{2} \sum_{i, j} a_{i j} p_{i} p_{j}, \quad i, j, l=1, \ldots, m .
\end{aligned}
$$

The reason for introducing the ordinary differential equations (C4) Is that it is possible to get a solution $v(\vec{x})$ to (C3) by solving instead the set (C4). In order to do so we must convert the boundary conditions 
on (C3) into initial conditions on (C4), as discussed below.

Boundary conditions on $v(\vec{x})$ are discussed in Chapter $V$ and may be specified as follows. We pick a surface very close to and surrounding the point $\vec{x}=\overrightarrow{0}$, and define the surface in terms of parameters $\vec{\theta}=$ $\left(\theta_{1}, \theta_{2}, \ldots, \theta_{m-1}\right)$ by equations $\vec{x}=\vec{x}(\vec{\theta})$. On the surface $\vec{x}(\vec{\theta})$ the behavior of $v(\vec{x})$ is defined by equation (5.13). We therefore obtain the quantities $v(\vec{\theta}), \vec{p}(\vec{\theta}), \vec{x}(\vec{\theta})$, which are such that $E[\vec{x}(\vec{\theta}), \vec{p}(\vec{\theta}), v(\vec{\theta})]=0$ identically. This set of functions is used as initial conditions for the ordinary differential equations (C4), 1.e., we set $\vec{x}(\sigma=0)=\vec{x}(\vec{\theta})$, $\vec{p}(\sigma=0)=\vec{p}(\vec{\theta}), v(\sigma=0)=v(\vec{\theta})$. For every value of $\vec{\theta}$, or equivalently for every point on the starting surface, we then solve (c4) to get a solution curve $\vec{x}(\sigma, \vec{\theta}), \vec{p}(\sigma, \vec{\theta}), v(\sigma, \vec{\theta})$. From (C4) we see that $\frac{d E}{d \sigma}=0$ and therefore $E=0$ along every solution curve. Now if the Jacobian $J=\frac{\partial\left(x_{1}, x_{2}, \ldots, x_{m}\right)}{\partial\left(\sigma, \theta_{1}, \ldots, \theta_{m-1}\right)} \neq 0$, we can write $\sigma, \vec{\theta}$ as functions of $\vec{x}$ along the solution curves, and insert these functions into $v(\sigma, \vec{\theta})$ to obtain $v(\vec{x})$. From the third equation in (C4) it follows that $v$ is increasing or nondecreasing on every solution curve. Practical implementation of this procedure is illustrated by the work of Cohen and Lewis (1967) and Ludwig (DL 75).

Once $v(\vec{x})$ has been found we return to the second term in (C2) and determine $h(\vec{x})$. As a preliminary step, we use the first equation of. (C4) to obtain the following equation concerning the rate of change of the Jacobian $\mathrm{J}$ along the solution curves,

$$
\frac{\mathrm{d} \ln \mathrm{J}}{\mathrm{d} \sigma}=\sum_{i} \frac{\partial}{\partial \mathrm{x}_{i}}\left(\frac{\mathrm{dx}}{\mathrm{d} \sigma}\right), \quad i=1, \ldots, \mathrm{m} .
$$


Using (C5) and the equation $\frac{d h}{d \sigma}=\sum_{1} \frac{d x_{1}}{d \sigma} \frac{\partial h}{\partial x_{1}}$, the terms in the second curly bracket in (C2) can be rewritten and set to zero to give the following equation for $h(\vec{x})$,

$$
\begin{aligned}
\frac{d}{d \sigma}(2 \ln h+\ln J)= & \sum_{i}\left(C_{i} \frac{\partial v}{\partial x_{i}}-\frac{\partial G_{i}}{\partial x_{i}}\right)-\sum_{i, j} \frac{\partial a_{i j}}{\partial x_{i}} \frac{\partial v}{\partial x_{j}} \\
& i, j=1, \ldots, m .
\end{aligned}
$$

Finally we set out the details of the formula for $\lambda_{0}$, $1 . e .$, equation (5.11). An important technical point is that the density for the $\overrightarrow{\mathrm{x}}(t)$ process is zero on the boundary $\Sigma$. To leading order in $\varepsilon$ the formula for $\lambda_{0}$ (obtained by integrating both sides of $(5.8)$ ) is

$$
\lambda_{0}=\frac{\sum_{i, j} \int_{\sum} \frac{1}{2} a_{i j} \frac{\partial v}{\partial x_{j}} h \exp \left(-\frac{v}{\varepsilon}\right) d S_{i}}{\iint g(\vec{x}) d \vec{x}}
$$

The numerator of (C7) is a surface integral with $\mathrm{dS}_{i}$ being area elements of $\Sigma$, while the denominator is a normalization integral over $\vec{x}-$ space. Let $\Sigma^{*}$ be the point on $\Sigma$ where $v(\vec{x})$ is a minimum, and introduce local orthonormal coordinates $\eta_{1}, n_{2}, \ldots, \eta_{m-1}$ on $\Sigma$ near $\Sigma^{*}$, as well as a coordinate $\eta_{m}$ normal to $\Sigma$ at $\Sigma^{*}$. Then (C7) may be estimated in terms of the principal contribution from the neighborhood of $\Sigma^{*}$, giving 


$$
\begin{aligned}
& \lambda_{0}=K\left(\Sigma^{*}\right) \exp \left[-\frac{v\left(\Sigma^{*}\right)}{\varepsilon}\right] \text {, }
\end{aligned}
$$

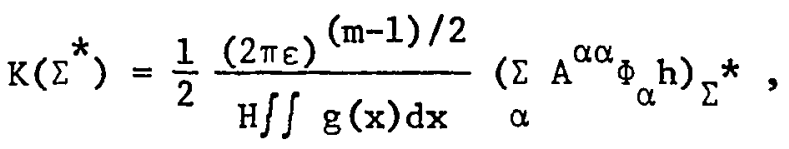

$$
\begin{aligned}
& A^{\alpha \beta}=\sum_{i, j} a_{i j} \frac{\partial \eta_{\alpha}}{\partial x_{i}} \frac{\partial \eta_{\beta}}{\partial x_{j}} \\
& \Phi_{\alpha}=\sum_{i} \frac{\partial v}{\partial x_{i}} \frac{\partial x_{i}}{\partial \theta_{\alpha}} \\
& H^{2}=\operatorname{det} \frac{\partial^{2} v}{\partial \theta_{\alpha} \partial \theta_{\beta}} \Sigma^{*}
\end{aligned}
$$

i, $j, \alpha, \beta=1, \ldots, m$.

The subscripts $\Sigma^{*}$ in the equations of (C8) indicate that the terms in the bracket are evaluated at the point $\Sigma^{*}$. It is at $\Sigma^{*}$ that the process $\vec{X}(t)$ is most likely to reach $\Sigma$ (DL 75, Vent'sel and Friedlin 1970). 